\title{
COORDINATING A FRESH-PRODUCT SUPPLY CHAIN WITH DEMAND INFORMATION UPDATING: HEMA FRESH O2O PLATFORM
}

\author{
Honglin YANG AND JiAWU PENG*
}

\begin{abstract}
Motivated by Hema Fresh's new-retail case, we study the coordination of a two-echelon fresh-product supply chain consisting of a single supplier and a single retailer. Due to a long production lead time, the supplier has to make production decision in advance based on early demand information. The market demand can be updated during the supplier's production lead time. Hence, the retailer would make order decision according to the latest demand information. Incorporating risk-sharing mechanism of overproduction and overstock, we propose a novel bi-directional risk-sharing contract to coordinate such a supply chain with demand information updating. We construct a two-stage optimization model in which the supplier first decides production quantity, and then the retailer decides final order quantity not exceeding the supplier's initial production. In both the centralized and decentralized systems, we analytically derive the unique equilibrium of production and order decisions in a Stackelberg supplier-led game. We prove that the proposed contract can realize supply chain perfect coordination and explore how the proposed contract affects the members' decisions. The theoretical results show that, by turning the risk-sharing proportions, the supply chain profit can be arbitrarily split between the members, which is a desired property for supply chain coordination. Compared with the single risk-sharing contract, the proposed contract results in a greater supply chain profit and achieves Pareto improvement for both members. Furthermore, we also explore how the risk preference and negotiating power affect the contract selection and the additional profit allocation of the supply chain. Numerical examples are presented to verify our theoretical results.
\end{abstract}

Mathematics Subject Classification. 35J20, 35J25, 35J60.

Received July 13, 2020. Accepted February 17, 2021.

\section{INTRODUCTION}

In real-world business, the mismatch of supply and demand leads to the occurrence of overproduction and overstock and further seriously hurts supply chain performance. Many high-tech manufacturers declared bankruptcy due to sharp demand shortfall [50]. Hence, how to utilize market information to effectively match supply and demand has drawn widespread attention in the academics and practitioners. Currently, numerous enterprises are actively collecting and employing market information to improve their decision-makings. For example, the web logs of Amazon gather more than 30 GB data every day [17]. Walmart's commercial data warehouse records

Keywords. Supply chain management, demand information updating, dynamic order, supply chain coordination, bi-directional risk-sharing contract.

School of Business Administration, Hunan University, Changsha, Hunan 410082, P.R. China.

*Corresponding author: jiawu_peng@163.com 


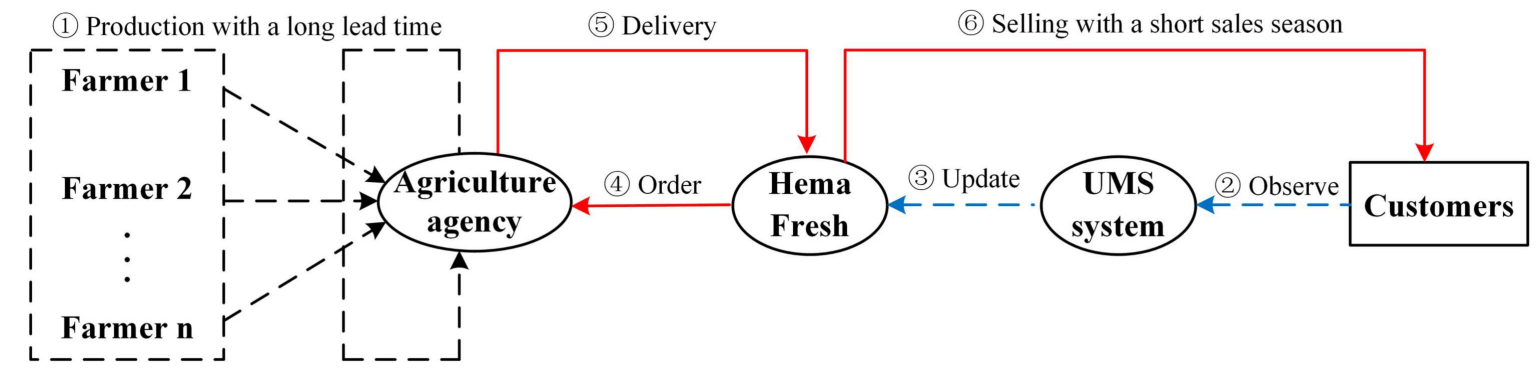

FiguRE 1. Fresh product's production and order in Hema Fresh's O2O platform.

more than 100 TB data for 65 weeks of historical transactions [26]. The supply chain enterprises also are focusing on demand information updating to cut down the cost of inventory. For instance, many fashion companies such as Uniqlo, Zara and Topshop, have adopted the quick response system to reduce market demand uncertainty $[23,28]$. As a result, the production capacity of the upstream supplier and the sales quantity of the downstream retailer are closer to real market demand $[12,31]$.

Although demand information updating may benefit the supply chain, the supply chain enterprises often act as self-interested members to maximize their own profit and minimize individual risk. Specifically, the retailer always prefers to postpone order before sales season until the demand information is improved. Doing that, the retailer may flexibly deal with market volatility to avoid the potential cost of overstock. However, the supplier tends to the situations that the retailer's order can be placed as early as possible so that it can hedge against the costs of over- and under-production [49]. At the same time, the supply chain member's risk preference and negotiating power also affect the supply chain decision-makings. For example, a fresh product supplier often suffers the risk of surplus due to high demand uncertainty, long supply lead time and short product-life cycle. Lack of the compensation, the risk-averse supplier would act conservatively to shed the potential overproduction risk [9]. Many scholars have noticed that demand information updating always benefits the retailer being close to a market while may hurt the supplier in certain cases $[42,47]$. To occupy more supply chain profit, the selfinterested supplier with strong negotiating power would prevent the retailer from modifying order plans after demand information updating. Therefore, how to mitigate the double marginal effects to create greater supply chain profit still is a big challenge in the current supply chain management.

These conflicts among channel members result in an inefficient supply chain, which cannot be avoided by demand information updating [2,3]. Take the fresh product supply chain in the Hema Fresh new-retail platform for example. Such as Yangcheng Lake hairy crabs, a specialty in Suzhou, Jiangsu Province of China, have a production lead time of more than 9 months but a sales season less than 3 months. As production lead time is long, crab farmers have to produce in advance. However, huge capacity investment cost, high demand uncertainty and short-lifecycle in crab industry increase the risk of surplus capacity for crab farmers. To reduce the overproduction risk, local crab farmers establish an agriculture agency as their representative to make a unified production plan. Meanwhile, to quickly respond to market changes and avoid overstock, Hema fresh builds an UMS system to collect and analyze market information during the long production season. Before the sales season, the UMC system updates market information and sends a procurement plan to Hema. Then, Hema negotiates with agriculture agency to sign an order contract. Due to complex breeding environment, the annual output of Yangcheng Lake hairy crabs is about 20 tons. Hence, Hema Fresh only can place an order of crabs to the agriculture agency no more than 20 tons, which severely limits Hema's order decision-making. Figure 1 illustrates fresh products' production and order in Hema Fresh.

These characteristics of fresh product and personalized needs of consumers expose both the supplier and retailer to a high level of inventory risk. Therefore, how to efficiently update demand information to satisfy customers' demand and further create greater supply chain profit is becoming a big challenge that Hema Fresh 
faces. Motivated by Hema Fresh new-retail platform, we consider a two-echelon fresh-product supply chain in which the supplier needs a long lead time to produces in advance relaying on early demand information and the retailer makes final order upon updating demand information. We design a new bi-directional risk-sharing contract to investigate how to coordinate such a supply chain with demand information updating.

We formulate a two-stage dynamic optimization decision-making model to depict Hema Fresh's new-retail case in which the supplier prepares fresh products in advance according to early demand information in the first stage, and the retailer makes final order upon observing later demand information in the second stage. The fresh products need a long production lead time to produce, so there will be no replenishment opportunity in the sales season. The retailer's final order quantity does not exceed the supplier's initial production quantity. The uncertain demand likely results in the retailer's overstock and the supplier's overproduction. The supply chain members would reduce production quantity or order quantity to avoid the potential loss risk. To mitigate the double marginal effects, we propose a new bi-directional risk-sharing contract to coordinate such a fresh-product supply chain with demand information updating. The proposed contract possesses two desirable features. First, it's superior to the conventional single risk-sharing contracts (i.e., overproduction risk-sharing contract or overstock risk-sharing contract). It not only allows the retailer to place a more accurate order according to the latest demand information but also can effectively mitigate the double marginal effects. Second, the proposed contract form is flexible, i.e., the supply chain profit can be arbitrarily split between members by turning the proportions of risk-sharing, which is a desired property for the supply chain coordination. We have also studied the effects of supply chain members' risk preferences and negotiating powers on the issue of the selection for the coordination contract.

This paper contributes to the existing literatures on risk-sharing contract and demand information updating in following three main aspects: first, to the best of our knowledge, this paper is the first to propose a bidirectional risk-sharing contract involving risks of overproduction and overstock to coordinate a two-echelon supply chain with demand information updating. Comparatively, most existing literatures only focus on single risk-sharing contracts for over-/under production risk (e.g., $[20,36])$ or overstock risk (e.g., [24]). Through redesigning the contract parameters, those papers explore the sufficient condition in which the supply chain achieves coordination. Second, we relax the related assumptions in the existing literatures of inventory model with demand information updating to consider a more general coordination contract from the risk-sharing perspective in a supplier-lead supply chain with a long production-lead time and "make-to-order" policy. Thus, the settings in this paper is more in line with the real business situation of fresh product. Third, with a bidirectional risk-sharing contract, we analytically derive the supply chain Pareto improvement region in which the supply chain not only achieves the system-optimal profit but also makes both members better off than the single risk-sharing contract. In addition, we also investigate how the supply chain members' risk preferences and negotiating powers affect the selection for the coordination contract.

The remainder of this paper is organized as follows. Section 2 reviews the related literature. Section 3 presents the model. Section 4 analyzes quantity decisions in the centralized and decentralized system, respectively. Section 5 investigates the design of coordination mechanism for the proposed contract. Section 6 explores the influences of the risk preference and negotiating power. Section 7 discusses numerical examples and sensitivity analysis. Section 8 concludes and presents main management insights.

\section{LiteratuRE REVIEW}

This paper mainly contributes to two streams of related literatures in the supply chain management: supply chain contract coordination with demand information updating and supply chain inventory model with risksharing. In what follows, we present a concise review and add more discussion to highlight the differences and similarities of our research compared with the reviewed literatures. 


\subsection{Supply chain contract coordination with demand information updating}

In the past decades, the design of supply chain contracts has brought a broad appreciation for the inventory model in the context of demand information updating [33]. To encourage both the supplier and the retailer to act in the manner that reaches system-optimal level, many classic supply chain contracts (e.g., wholesaleprice, return, revenue-sharing, commitment and quantity flexibility, etc.) have been developed and improved to achieve channel coordination. Prior researches have focus on how the demand information updating affects the realization of supply chain coordination. There are two main research streams in these literatures. In the first stream, demand information updating occurs in the sales season in a two-period modeling framework. Under this stream, the retailer can use the realized demand in the first period to update the demand forecast in the second period (see $[4,6,7,34,50,51]$ ). For example, Barnes-Schuster et al. [4] investigate the role of commitmentoption in a retailer-supplier system using a two-period model with correlated demand. They prove that the supply chain coordination can be achieved only if the exercise price to be piecewise linear. Zhou and Wang [51] study supply chain coordination for newsvendor-type products with two ordering opportunities and demand information updating. They find out that revenue-sharing contract fails to coordinate such a supply chain. To realize the perfect coordination of the supply chain, they propose an improved revenue-sharing contract in that the supplier not only shares the retailer's revenue but also bears a portion of the retailer's operating costs.

In the second stream, demand information updating occurs in the production season for fashion products with short lifecycles and long lead time. Under this stream, the market signals during the production season can be collected to improve the demand forecast in the sales season (see [5, 8-15, 29,32,37,42]). For example, Chen et al. [9] investigate the coordination of a supply chain with demand information updating. They demonstrate that the conventional return contract should be modified to achieve supply chain coordination. Wu [42] considers a two-echelon supply chain consisting of a single supplier and a single retailer with Bayesian update. Under a quantity flexibility contract, the results show that the retailer always benefits from flexibility, whereas the supplier only benefits when the level of flexibility is small. Choi [11] studies the influence of the inventory service target on quick response fashion retail supply chain. The analysis results show that the wholesale price contract achieves win-win in a fashion retail supply chain after implementing quick response only when the inventory service target is higher than 0.5 .

To quick response the market change, above researches build on that the upstream supplier can fulfill all the downstream retailer's instant orders immediately. There exist two main assumptions to ensure the supplier acts in that manner. First, they assume that the supplier adopts "make-to-order" production policy in a retailerlead supply chain. Under this setting, the demand information updating benefits the retailer but hurt the supplier due to overproduction. To achieve coordination, all of above researches added a transfer payment from the retailer to the supplier into the conventional contracts to sharing the overproduction risk. This means that the improved coordination contracts may not be individually rational for the supplier. Second, they assume that the supplier has a full production capacity. However, for fresh product such as vegetables, fruits and seafood, the supplier's capacity is often limited due to a long production lead time and random yield. In addition, in a twoechelon supply consisting of a single supplier and a single retailer, there is no replenishment opportunity for the sales season. Therefore, the retailer's order quantity cannot exceed the supplier's initial production quantity. By relaxing the above assumptions, we consider a more general coordination contract from the risk-sharing perspective in a supplier-lead supply chain with a long production-lead time and demand information updating.

\subsection{Supply chain inventory model with risk-sharing}

Due to the rapid development of technology, demand information can be easily collected and updated for improving supply chain performance. However, no matter how good the employed forecasting technique is, it is almost impossible to provide accurate forecast subject to demand uncertainty [24]. Considering that the uncertain demand always results in a situation where both the supplier and retailer face the risk of over- or out-inventory, risk-sharing contract is one of the means of risk mitigation [16, 25,39]. Two classes of supply chain risk-sharing models exist. In the first class, only the order risk is sharing between the supplier and the 
TABLE 1. Comparisons between this paper and the related literatures.

\begin{tabular}{lllll}
\hline \hline Literature & Model & Stackelberg game & Risk-sharing & $\begin{array}{l}\text { Demand } \\
\text { information } \\
\text { updating }\end{array}$ \\
\hline $\begin{array}{l}\text { He and Zhang } \\
{[20]}\end{array}$ & Make-to-order & Retailer-lead & Over or under-production & No-updating \\
Kim [24] & Make-to-order & Retailer-lead & Overstock & Updating \\
$\begin{array}{l}\text { Tang and } \\
\text { Kouvelis [36] }\end{array}$ & Make-to-order & Retailer-lead & Overproduction & No-updating \\
$\begin{array}{l}\text { This paper } \\
\text { (2013) }\end{array}$ & Make-to-stock & Supplier-lead & Overproduction or overstock & Updating \\
\hline
\end{tabular}

retailer, while the production risk is fully carried by the supplier (see $[1,24,27,40,44,46]$ ). For example, Li and Kouvelis [27] develop a risk-sharing sourcing contract with time, quantity, and supply flexibility. They find that contractual flexibility in sourcing arrangements can effectively reduce the sourcing cost in environments of price uncertainty. Wang and Liu [40] present an option contract model to analyze the coordination and risk sharing problems in a retailer-led supply chain in which the powerful retailer takes the initiative to coordinate the manufacturer's production quantity. Kim [24] studies a bilateral contract with order quantity flexibility as a risk-sharing mechanism for demand forecasting in a supply chain, in which the retailer places orders in advance for the predetermined horizons and makes minimum purchase commitments. The supplier provides the retailer with the flexibility to adjust the order quantities later according to the most updated demand information. The numerical examples show that demand fluctuation can be effectively absorbed by the contract scheme, which enables better inventory management and customer service. Adhikari et al. [1] represent the textile supply chain using a five-level supply chain structure under simultaneous demand and supply uncertainty. They show how a risk-sharing contract improves the profitability of the cotton firm in a highly loss-making scenario.

Furthermore, in the second class, only the production risk is sharing between the supplier and the retailer, while the order risk is fully carried by the retailer (see [19,20,22,36,43,48]). For example, He and Zhang [20] consider the production and order decisions in a two-echelon supply chain with random yield and uncertain demand. They proposed several risk-sharing contracts that distribute the over- or under-production risk among the supply chain members. Inderfurth and Clemens [22] study how a risk-sharing mechanism affects the retailer's order decision and the supplier's production decision with random yield and deterministic demand. They prove that the wholesale price contract will guarantee supply chain coordination if the supplier has an emergency procurement source that is more costly but reliable. Zare et al. [48] consider a supply chain in which a supplier faces random yield and multiple retailers deal with random demands. In a Stackelberg game structure, the effects of demand uncertainty, production costs' sharing and wholesale price reduction approaches are explored. They find that risk-sharing contract may significantly reduce supplier's holding costs, which benefits both the supplier and the retailers in comparison with no-risk-sharing contract.

However, to the best of our knowledge, most of the reviewed literatures only focus on the production risk or the order risk, and there are few literatures considering both the production and order risks. Therefore, our work contributes to the literatures by considering a bi-directional risk-sharing contract for both overproduction risk and overstock risk in a two-echelon supply chain. In particular, we study the supplier's production decision and the retailer's order decision in the context of demand information updating.

Our work is close in spirt and structure to that of He and Zhang [20], Kim [24] and Tang and Kouvelis [36]. However, there exists following noticeable difference between their work and our study (see Tab. 1). First, we distribute both the overproduction risk and overstock risk among the supply chain members using a bidirectional risk-sharing contract. Specifically, in our model, the supplier's production quantity is inconsistent with the retailer's order quantity due to demand information updating. To eliminate the double marginal 
effects, we introduce a novel bi-directional risk-sharing contract in which the retailer would share a part of the overproduction risk with the supplier at the end of production season and the supplier would share a part of the overstock risk with the retailer at the end of sales season. Second, in our model, the supplier need produce in advance due to a long production lead time, while the retailer can delay order until a more accurate demand information is available. Hence, we consider a Stackelberg game problem in which the supplier can be viewed as a leader while the retailer is viewed as a follower. Third, from the risk-sharing's perspective, we address the coordination issue in a two-echelon supply chain with demand information updating. We find that the single risk-sharing contract may be non-individually rational for the supplier, while the bi-directional risk-sharing contract can make both the supplier and the retailer better off than non-risk-sharing contract. In addition, we further study the effects of supply chain members' risk preferences and negotiating powers on the issue of the selection for the coordination contract.

\section{MODEL DESCRIPTION}

We consider a two-echelon supply chain in which the retailer orders fresh product from the supplier and then sells it to end-customers. For convenience, the supplier is referred as "he" and the retailer as "she". The supply chain members face a stochastic market demand, which can be updated using market signals or observations. Following Gurnani and Tang [18], the process of demand information updating is expressed as follows. At the beginning of production season, the retailer estimates that early demand information, denoted by $x$, has $a$ prior probability distribution $F(x)$ and a density function $f(x)$. The retailer can utilize the market signal $I$ observed during the production season to update the probability distribution of $x$, where $G(I)$ and $g(I)$ denote the marginal distribution and density of $I$. At the end of production season, the latest market signal is realized as $I=i$, and the latest demand information, denoted by $x \mid i$, has a posterior distribution $F(x \mid i)$ and density function $f(x \mid i)$. Assume that the distribution $F(x \mid i)$ is strictly increasing in $x$ for given $i$ and all distributions are continuous, differentiable, and invertible. Many conjugate distributions can satisfy the above assumptions, such as uniform-uniform, uniform-Pareto, beta-negative binomial, gamma-Poisson and normal-normal distributions. To illustrate, suppose that market demand follows the Uniform-Uniform distribution $[9,21,41]$. Then, the new demand signal $I$ and posterior demand $x \mid i$ with $I=i$ are given as follows:

$$
\begin{aligned}
g(I) & =\frac{1}{\alpha} \text { and } G(i)=\frac{1}{\alpha}\left(I-\gamma+\frac{\alpha}{2}\right), \text { where } I \in\left[\gamma-\frac{\alpha}{2}, \gamma+\frac{\alpha}{2}\right] \\
f(x \mid i) & =\frac{1}{\beta} \text { and } F(x \mid i)=\frac{1}{\beta}\left(x-i+\frac{\beta}{2}\right), \text { where } x \in\left[i-\frac{\beta}{2}, i+\frac{\beta}{2}\right] .
\end{aligned}
$$

In the above demand setting, the supply chain's problem is described as a two-stage optimization problem: (1) At the beginning of Stage 1 (i.e., production season), the supplier decides production quantity $Q_{s}^{j}$ at a unit cost $c$ based on early demand information $f(x)$. After observing new market signal at the end of Stage 1 , the retailer decides the order quantity $Q_{r}^{j}$ according to the latest demand information $f(x \mid i)$ and pays a unit wholesale price $w$ to the supplier. The unit salvage value of leftovers that has not been ordered by the retailer is $v_{s}$. If $Q_{r}<Q_{s}$, the supplier has a loss of $\left(c-v_{s}\right)\left(Q_{s}^{j}-Q_{r}^{j}\right)$ due to overproduction risk. (2) At the beginning of Stage 2 (i.e., sales season), the retailer sells fresh product to customers at a fixed retail price $p$. The unit salvage value of leftovers that has not been purchased by end-customers is $v_{r}$. At the end of Stage 2, the retailer may face a loss of $\left(w-v_{r}\right)\left(Q_{r}-x\right)^{+}$due to overstock risk. To avoid trivial or non-realistic cases, we assume that $p>w>c>v_{s}>v_{r}$. Besides, we further assume that the retailer's demand information and the supplier's production quantity are shared between two channel members.

Moreover, a novel bi-directional risk-sharing contract is introduced to coordinate a two-echelon fresh-product supply chain in the context of demand information updating. To be more specific, at the end of production season, the retailer commits a fixed proportion $\rho \in[0,1]$ to share the supplier's loss due to overproduction risk, i.e., $\rho\left(c-v_{s}\right)\left(Q_{s}^{j}-Q_{r}^{j}\right)$. Then, at the end of sales season, the supplier also commits a fixed proportion $\phi \in[0,1]$ to share the retailer's loss due to overstock risk, i.e., $\phi\left(w-v_{r}\right)\left(Q_{r}^{j}-x\right)^{+}$. In the above commit setting, the 


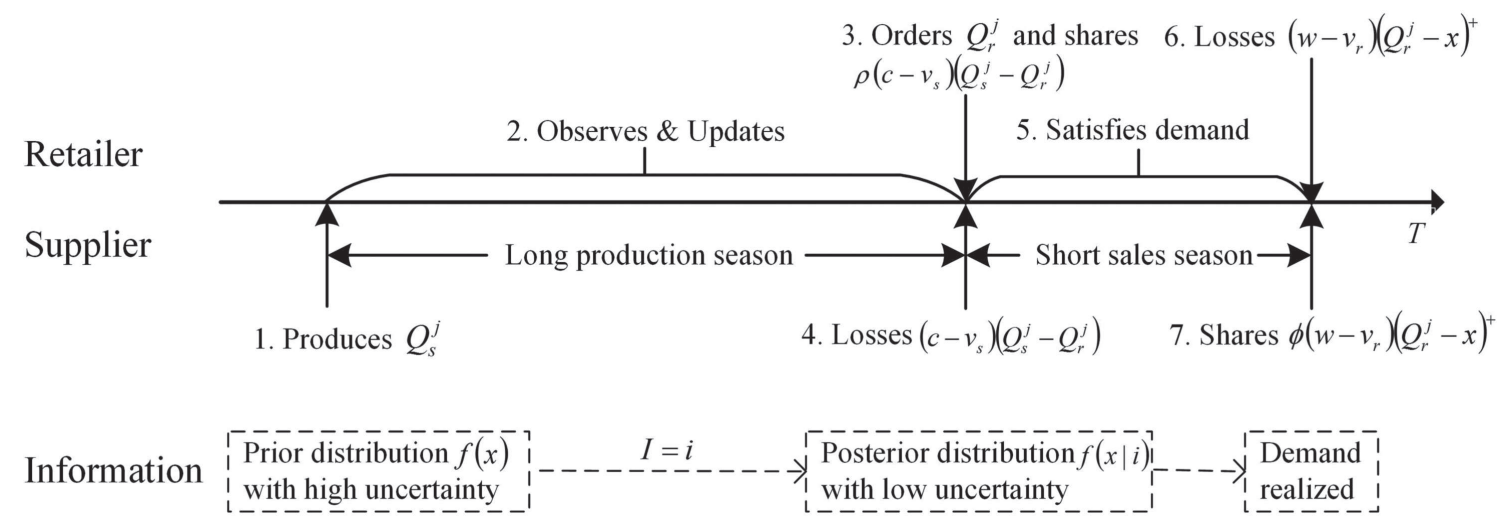

Figure 2. The time line of the events.

TABLE 2. Notations and explanations.

\begin{tabular}{ll}
\hline \hline Notation & Explanation \\
\hline$x$ & Market demand \\
$I$ & Market signal \\
$i$ & Realize value of market signal \\
$w$ & Wholesale price \\
$p$ & Retail price \\
$c$ & Production cost \\
$v_{s}$ & Salvage value per unit overproduction \\
$v_{r}$ & Salvage value per unit overstock \\
$\rho$ & Sharing proportion of overproduction \\
$\phi$ & Sharing proportion of overstock \\
$Q_{s}^{j}$ & Production quantity of the supplier (decision variable) \\
$f(x), F(x)$ & Prior probability density and distribution functions of $x$ \\
$g(I), G(I)$ & Salvage value of leftover inventory \\
$f(\cdot), F(\cdot)$ & Probability density and distribution functions of $I$ \\
$f(x \mid i)$ & Posterior probability density function of $x$ given $I=i$ \\
$F(x \mid i)$ & Posterior probability distribution function of $x$ given $I=i$ \\
$\Pi^{j}$ & Profit function \\
$\pi^{j}$ & Expected function \\
\hline
\end{tabular}

proposed contract, denoted by $\{w, \rho, \phi\}$, permits any agreed-upon division of the overproduction and overstock risks between the supplier and the retailer. Figure 2 illustrates the sequence of events in such a supply chain.

In what follows, we study the supplier's production decision and the retailer's ordering decision with the above bi-directional risk-sharing mechanism. In particular, we construct four game-theoretic decision-making models to analysis the effects of risk-sharing proportion on the supply chain members' behaviors, which are denoted as: (1) NRS model (i.e., No risk-sharing model); (2) ORS-1 model (i.e., Overproduction risk-sharing model); (3) ORS-2 model (i.e., Overstock risk-sharing model); (4) BRS model (Bi-directional risk-sharing model). It is worth mentioning that the NRS model, ORS-1 model and ORS-2 model are special cases of BRS model. Furthermore, the subscripts $j=\mathrm{SC}, N, S 1, S 2, B$ denote the centralized model, NRS model, ORS-1 model, ORS-2 model and BRS model respectively.

For easy interpretation, the key notations are listed in Table 2. 


\section{QUANTITY DECISIONS ANALYSIS}

\subsection{Benchmark: centralized model}

In the centralized model, the supplier and the retailer belong to the same company. The supply chain first determines production quantity $Q_{s}^{S C}$ based on early demand information at the beginning of Stage 1 . Then, the supply chain determines order quantity $Q_{r}^{\mathrm{SC}}$ utilizing the latest demand information at the beginning of Stage 2 (this ordering quantity can be thought of the amount of fresh product that actually ship to the market). Using the backward induction method, we solve this two-stage optimization model with demand information updating as follows.

First, at the beginning of Stage 2, given $Q_{s}^{\mathrm{SC}}$ and $I=i$, the supply chain's optimal ordering quantity $Q_{r}^{\mathrm{SC}}$ is obtained by solving the optimization problem (P1).

$$
\begin{array}{ll}
\underset{Q_{r}^{\mathrm{SC}}}{\operatorname{maximize}} & \Pi^{\mathrm{SC}}\left(Q_{s}^{\mathrm{SC}}, i ; Q_{r}^{\mathrm{SC}}\right) \\
\text { subject to } & Q_{r}^{\mathrm{SC}} \leq Q_{s}^{\mathrm{SC}}
\end{array}
$$

where $\Pi^{\mathrm{SC}}\left(Q_{s}^{\mathrm{SC}}, i ; Q_{r}^{\mathrm{SC}}\right)=E_{\{x \mid i\}}\left\{p \min \left(x, Q_{r}^{\mathrm{SC}}\right)+v_{r}\left(Q_{r}^{\mathrm{SC}}-x\right)^{+}+v_{s}\left(Q_{s}^{\mathrm{SC}}-Q_{r}^{\mathrm{SC}}\right)\right\}$.

Let $Q_{r}^{\mathrm{SC}} \mid i=F^{-1}\left(\frac{p-v_{s}}{p-v_{r}}\right)$, where $F^{-1}(\cdot)$ is the inverse function of $F(\cdot)$. Note that $Q_{r}^{\mathrm{SC}} \leq Q_{s}^{\mathrm{SC}}$, the following proposition establishes the optimal order quantity in the centralized model.

Proposition 4.1. Given $Q_{s}^{\mathrm{SC}}$ and $I=i$ in Stage $2, \Pi^{\mathrm{SC}}\left(Q_{s}^{\mathrm{SC}}, i ; Q_{r}^{\mathrm{SC}}\right)$ is concave in $Q_{r}^{\mathrm{SC}}$, and

$$
Q_{r}^{\mathrm{SC}}= \begin{cases}Q_{r}^{\mathrm{SC}} \mid i, & \text { if } i \leq i_{Q_{s}^{\mathrm{SC}}}, \\ Q_{s}^{\mathrm{SC}}, & \text { if } i>i_{Q_{s}^{\mathrm{SC}}}\end{cases}
$$

where $i_{Q_{s}^{\mathrm{SC}}}$ satisfies $Q_{r}^{\mathrm{SC}} \mid i_{Q_{s}^{\mathrm{SC}}}=Q_{s}^{\mathrm{SC}}$.

When market demand follows the uniform-uniform distribution, an equivalent form of Proposition 4.1 is given as follows.

Corollary 4.2. Given $Q_{s}^{\mathrm{SC}}$ and $I=i$ in Stage 2 , there is a positive threshold $i_{Q_{s}^{\mathrm{SC}}}=Q_{s}^{\mathrm{SC}}+\frac{\beta}{2}-\frac{p-v_{s}}{p-v_{r}} \beta$ such that

$$
Q_{r}^{\mathrm{SC}}= \begin{cases}Q_{r}^{\mathrm{SC}} \mid i=i-\frac{\beta}{2}+\frac{p-v_{s}}{p-v_{r}} \beta, & \text { if } \gamma-\frac{\alpha}{2} \leq i \leq i_{Q_{s}^{\mathrm{SC}},} \\ Q_{s}^{\mathrm{SC}}, & \text { if } i_{Q_{s}^{\mathrm{SC}}}<i \leq \gamma+\frac{\alpha}{2} .\end{cases}
$$

From Proposition 4.1, the supply chain's optimal order quantity $Q_{r}^{\mathrm{SC}}$ depends on the production quantity and updated demand information. To be more specific, if $i \leq i_{Q_{s}^{\mathrm{SC}}}$, then $Q_{r}^{\mathrm{SC}}=Q_{r}^{\mathrm{SC}} \mid i \leq Q_{s}^{\mathrm{SC}}$, which indicates that the supply chain's production is sufficient and some fresh product are not shipped to market. If $i>i_{Q_{s}^{\mathrm{SC}}}$, then $Q_{r}^{\mathrm{SC}}=Q_{s}^{\mathrm{SC}} \leq Q_{r}^{\mathrm{SC}} \mid i$, which indicates that the supply chain's production is insufficient and there is no fresh product surplus. Based on above discussion, the supply chain's optimal expected profit during Stage 2 is

$$
\Pi^{\mathrm{SC}}\left(Q_{s}^{\mathrm{SC}}, i ; Q_{r}^{\mathrm{SC}}\right)= \begin{cases}\Pi^{\mathrm{SC}}\left(Q_{s}^{\mathrm{SC}}, i ; Q_{r}^{\mathrm{SC}} \mid i\right), & \text { if } i \leq i_{Q_{s}^{\mathrm{SC}}}, \\ \Pi^{\mathrm{SC}}\left(Q_{s}^{\mathrm{SC}}, i ; Q_{s}^{\mathrm{SC}}\right), & \text { if } i>i_{Q_{s}^{\mathrm{SC}}}\end{cases}
$$

where

$$
\Pi^{\mathrm{SC}}\left(Q_{s}^{\mathrm{SC}}, i ; Q_{r}^{\mathrm{SC}} \mid i\right)=\left(p-v_{s}\right) Q_{r}^{\mathrm{SC}} \mid i-\left(p-v_{r}\right) \int_{0}^{Q_{r}^{\mathrm{SC}} \mid i} F(x \mid i) \mathrm{d} x+v_{s} Q_{s}^{\mathrm{SC}},
$$

and

$$
\Pi^{\mathrm{SC}}\left(Q_{s}^{\mathrm{SC}}, i ; Q_{s}^{\mathrm{SC}}\right)=p Q_{s}^{\mathrm{SC}}-\left(p-v_{r}\right) \int_{0}^{Q_{s}^{\mathrm{SC}}} F(x \mid i) \mathrm{d} x
$$


Then, at the beginning of Stage 1, takes the results of the above two scenarios into consideration, we can obtain the supply chain's optimal production quantity $Q_{s}^{\mathrm{SC}^{*}}$ by solving the optimization problem (P2).

$$
\begin{array}{ll}
\underset{Q_{s}^{\mathrm{SC}}}{\operatorname{maximize}} & \Pi^{\mathrm{SC}}\left(Q_{s}^{\mathrm{SC}}\right) \\
\text { subject to } & Q_{s}^{\mathrm{SC}}>0,
\end{array}
$$

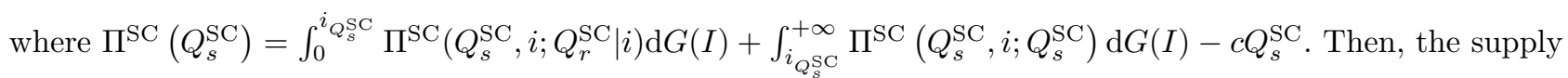
chain's optimal production quantity $Q_{s}^{\mathrm{SC}}$ is presented in following proposition.

Proposition 4.3. In stage $1, \Pi^{\mathrm{SC}}\left(Q_{s}^{\mathrm{SC}}\right)$ is concave in $Q_{s}^{\mathrm{SC}}$, and $Q_{s}^{\mathrm{SC}}$ is the unique solution of the following equation:

$$
\int_{i_{Q_{s}^{S C}}}^{+\infty}\left[F\left(Q_{r}^{\mathrm{SC}} \mid i\right)-F\left(Q_{s}^{\mathrm{SC}} \mid i\right)\right] \mathrm{d} G(I)=\frac{c-v_{s}}{p-v_{r}} .
$$

In addition, when the market demand follows the uniform-uniform distribution, $Q_{s}^{\mathrm{SC}^{*}}=\gamma+\frac{\alpha-\beta}{2}+\frac{p-v_{s}}{p-v_{r}} \beta-$ $\sqrt{\frac{2\left(c-v_{s}\right) \alpha \beta}{p-v_{r}}}$.

Proposition 4.3 characterizes the sufficient condition of the optimal production quantity in centralized supply chain. From the right-hand side of (4.4), the optimal production quantity $Q_{s}^{\mathrm{SC}^{*}}$ decreases in the general costprofit ratio (i.e., $\frac{c-v_{s}}{p-v_{r}}$ ). A smaller ratio means a lower production cost or larger marginal profit, which reduces the risk of overproduction and further induces the supplier to produce more. Unlike the classic supply chain inventory model with stochastic demand, we consider the supply chain members' quantity decisions model in the context of demand information updating, which are influenced by both overproduction risk and overstock risk in our model. This scenario is used as the benchmark for evaluating the performance of subsequent decentralized models.

\subsection{Decentralized model}

In the decentralized model, the supply chain members only act in their best interests, which results in a decentralized model extracting less profit than a centralized model. Furthermore, in this paper, there exists both overproduction risk and overstock risk due to demand information updating, which leads a more serious "double marginalization". To explore supply chain coordination mechanism from the risk-sharing perspective, we introduce four risk-sharing models in decentralized supply chain that consider both overproduction and overstock risks.

\subsubsection{No risk-sharing model (NRS)}

In the NRS model, the supplier does not share the overstock risk with the retailer and faces only the overproduction risk from demand information updating. Similarly, the retailer does not share the overproduction risk with the supplier and faces only the overstock risk from stochastic demand. In this setting, the supplier first decides his production quantity $Q_{s}^{N}$ at the beginning of Stage 1 . Then, the retailer decides her order quantity $Q_{r}^{N}$ at the beginning of Stage 2. Utilizing a backward induction method, we analytically derive the equilibrium of a Stackelberg game.

Given $Q_{s}^{N}$ and $I=i$, the retailer's optimal ordering quantity $Q_{r}^{N^{*}}$ at the beginning of Stage 2 is obtained by solving the optimization problem (P3).

$$
\begin{array}{ll}
\underset{Q_{r}^{N}}{\operatorname{maximize}} & \Pi_{r}^{N}\left(Q_{s}^{N}, i ; Q_{r}^{N}\right) \\
\text { subject to } & Q_{r}^{N} \leq Q_{s}^{N},
\end{array}
$$


where $\Pi_{r}^{N}\left(Q_{s}^{N}, i ; Q_{r}^{N}\right)=E_{\{x \mid i\}}\left\{p \min \left(x, Q_{r}^{N}\right)-w Q_{r}^{N}+v_{r}\left(Q_{r}^{N}-x\right)^{+}\right\}$.

Note that $Q_{r}^{N} \leq Q_{s}^{N}$, let $Q_{r}^{N} \mid i=F^{-1}\left(\frac{p-w}{p-v_{r}}\right)$, we obtain the retailer's optimal order quantity $Q_{r}^{N^{*}}$ of the NRS model in the following proposition.

Proposition 4.4. Given $Q_{s}^{N}$ and $I=i$ in Stage $2, \Pi_{r}^{N}\left(Q_{s}^{N}, i ; Q_{r}^{N}\right)$ is concave in $Q_{r}^{N}$, and

$$
Q_{r}^{N^{*}}= \begin{cases}Q_{r}^{N} \mid i, & \text { if } i \leq i_{Q_{s}^{N}} \\ Q_{s}^{N}, & \text { if } i>i_{Q_{s}^{N}}\end{cases}
$$

where $i_{Q_{s}^{N}}$ satisfies $Q_{r}^{N} \mid i_{Q_{s}^{N}}=Q_{s}^{N}$.

Similarly, if market demand follows the uniform-uniform distribution, Proposition 4.4 is rewritten as follows.

Corollary 4.5. Given $Q_{s}^{N}$ and $I=i$ in Stage 2 , there is a positive threshold $i_{Q_{s}^{N}}=Q_{s}^{N}+\frac{\beta}{2}-\frac{p-w}{p-v_{r}} \beta$ such that

$$
Q_{r}^{N^{*}}= \begin{cases}Q_{r}^{N} \mid i=i-\frac{\beta}{2}+\frac{p-w}{p-v_{r}} \beta, & \text { if } \gamma-\frac{\alpha}{2} \leq i \leq i_{Q_{s}^{N}}, \\ Q_{s}^{N}, & \text { if } i_{Q_{s}^{N}}<i \leq \gamma+\frac{\alpha}{2} .\end{cases}
$$

Proposition 4.4 shows that the retailer's optimal ordering quantity and its relation to the supplier's production quantity and updated demand information. Incorporating the two possible scenarios of the retailer's optimal ordering quantity, the supplier's optimal expected profit of NRS model in Stage 2 is

$$
\Pi_{s}^{N}\left(Q_{s}^{N}, i ; Q_{r}^{N^{*}}\right)= \begin{cases}\Pi_{s}^{N}\left(Q_{s}^{N}, i ; Q_{r}^{N} \mid i\right), & \text { if } i \leq i_{Q_{s}^{N}}, \\ \Pi_{s}^{N}\left(Q_{s}^{N}, i ; Q_{s}^{N}\right), & \text { if } i>i_{Q_{s}^{N}},\end{cases}
$$

with $\Pi_{s}^{N}\left(Q_{s}^{N}, i ; Q_{r}^{N} \mid i\right)=w Q_{r}^{N} \mid i+v_{s}\left(Q_{s}^{N}-Q_{r}^{N} \mid i\right)$ and $\Pi_{s}^{N}\left(Q_{s}^{N}, i ; Q_{s}^{N}\right)=w Q_{s}^{N}$.

Then, the supplier's optimal ordering quantity $Q_{s}^{N^{*}}$ at the beginning of Stage 1 is obtained by solving the optimization problem (P4).

$$
\begin{array}{cc}
\underset{Q_{s}^{N}}{\operatorname{maximize}} & \Pi_{s}^{N}\left(Q_{s}^{N}\right) \\
\text { subject to } & Q_{s}^{N}>0,
\end{array}
$$

where $\Pi_{s}^{N}\left(Q_{s}^{N}\right)=\int_{0}^{i_{Q_{s}^{N}}} \Pi_{s}^{N}\left(Q_{s}^{N}, i ; Q_{r}^{N} \mid i\right) \mathrm{d} G(I)+\int_{i_{Q_{s}^{N}}^{+\infty}}^{+} \Pi_{s}^{N}\left(Q_{s}^{N}, i ; Q_{s}^{N}\right) \mathrm{d} G(I)-c Q_{s}^{N}$.

The following proposition establishes the supplier's optimal production quantity.

Proposition 4.6. In stage $1, \Pi_{s}^{N}\left(Q_{s}^{N}\right)$ is concave in $Q_{s}^{N}$, and $Q_{s}^{N^{*}}$ satisfies $G\left(i_{Q_{s}^{N *}}\right)=\frac{w-c}{w-v_{s}}$. In addition, when market demand follows the uniform-uniform distribution, $Q_{s}^{N^{*}}=\gamma+\frac{\alpha-\beta}{2}+\frac{p-w}{p-v_{r}} \beta-\frac{c-v_{s}}{w-v_{s}} \alpha$.

\subsubsection{Overproduction risk-sharing model (ORS-1)}

In the ORS-1 model, the overproduction risk is shared between the supplier and the retailer, while the overstock risk is carried only by the retailer. In this setting, at the beginning of Stage 1, the supplier first decides his production quantity $Q_{s}^{S 1}$. At the beginning of Stage 2, the retailer decides her order quantity $Q_{r}^{S 1}$ when the latest demand information is available. If $Q_{r}^{S 1}<Q_{s}^{S 1}$, the retailer would pay $\rho\left(c-v_{s}\right)\left(Q_{s}^{S 1}-Q_{r}^{S 1}\right)$ to the supplier to share part of the overproduction risk. This problem is again a two-stage Stackelberg game decision problem.

Here, given $Q_{s}^{S 1}$ and $I=i$, the retailer's optimal ordering quantity $Q_{r}^{S 1^{*}}$ at the beginning of Stage 2 is obtained by solving the optimization problem (P5).

$$
\underset{Q_{r}^{S 1}}{\operatorname{maximize}} \Pi_{r}^{S 1}\left(Q_{s}^{S 1}, i ; Q_{r}^{S 1}\right)
$$




$$
\text { subject to } Q_{r}^{S 1} \leq Q_{s}^{S 1},
$$

where $\Pi_{r}^{S 1}\left(Q_{s}^{S 1}, i ; Q_{r}^{S 1}\right)=E_{\{x \mid i\}}\left\{p \min \left(x, Q_{r}^{S 1}\right)-w Q_{r}^{S 1}-\rho\left(c-v_{s}\right)\left(Q_{s}^{S 1}-Q_{r}^{S 1}\right)+v_{r}\left(Q_{r}^{S 1}-x\right)^{+}\right\}$.

Since $Q_{r}^{S 1} \leq Q_{s}^{S 1}$, let $Q_{r}^{S 1} \mid i=F^{-1}\left(\frac{p-w+\rho\left(c-v_{s}\right)}{p-v_{r}}\right)$, then the following proposition presents the retailer's optimal order quantity $Q_{r}^{S 1^{*}}$ of the ORS-1 model.

Proposition 4.7. Given $Q_{s}^{S 1}$ and $I=i$ in Stage $2, \Pi_{r}^{S 1}\left(Q_{s}^{S 1}, i ; Q_{r}^{S 1}\right)$ is concave in $Q_{r}^{S 1}$, and

$$
Q_{r}^{S 1^{*}}= \begin{cases}Q_{r}^{S 1} \mid i, & \text { if } i \leq i_{Q_{s}^{S 1}}, \\ Q_{s}^{S 1}, & \text { if } i>i_{Q_{s}^{S 1}}\end{cases}
$$

where $i_{Q_{s}^{S 1}}$ satisfies $Q_{r}^{S 1} \mid i_{Q_{s}^{S 1}}=Q_{s}^{S 1}$.

If market demand is the uniform-uniform distributed, Proposition 4.7 becomes following corollary.

Corollary 4.8. Given $Q_{s}^{S 1}$ and $I=i$ in Stage 2, there is a positive threshold $i_{Q_{s}^{S 1}}=Q_{s}^{S 1}+\frac{\beta}{2}-\frac{p-w+\rho\left(c-v_{s}\right)}{p-v_{r}} \beta$ such that

$$
Q_{r}^{S 1^{*}}= \begin{cases}Q_{r}^{S 1} \mid i=i-\frac{\beta}{2}+\frac{p-w+\rho\left(c-v_{s}\right)}{p-v_{r}} \beta, & \text { if } \gamma-\frac{\alpha}{2} \leq i \leq i_{Q_{s}^{S 1}}, \\ Q_{s}^{S 1}, & \text { if } i_{Q_{s}^{S 1}}<i \leq \gamma+\frac{\alpha}{2}\end{cases}
$$

Proposition 4.7 shows that the retailer's optimal ordering quantity in the ORS- 1 model is influenced by the supplier's production quantity, updated demand information and sharing proportion of overproduction. Specially, $Q_{r}^{S 1^{*}}$ increases in $\rho$. It indicates that, the higher sharing proportion of overproduction is, the more the retailer would order. That can be explained as follows: Given $Q_{s}^{S 1}$ and $I=i$, the amount that the retailer pays to the supplier to share overproduction loss is $\rho\left(c-v_{s}\right)\left(Q_{s}^{S 1}-Q_{r}^{S 1}\right)$, which increases with $\rho$ while decreases with $Q_{r}^{S 1}$. Hence, when making ordering decision, the retailer compares the possible losses from overproduction risk-sharing with overstock. As $\rho$ increases, it's beneficial for the retailer to order more to reduce the amount of $\rho\left(c-v_{s}\right)\left(Q_{s}^{S 1}-Q_{r}^{S 1}\right)$.

Knowing the retailer's optimal response, the supplier's optimal expected profit of ORS-1 model in Stage 2 is

$$
\Pi_{s}^{S 1}\left(Q_{s}^{S 1}, i ; Q_{r}^{S 1^{*}}\right)= \begin{cases}\Pi_{s}^{S 1}\left(Q_{s}^{S 1}, i ; Q_{r}^{S 1} \mid i\right), & \text { if } i \leq i_{Q_{s}^{S 1}}, \\ \Pi_{s}^{S 1}\left(Q_{s}^{S 1}, i ; Q_{s}^{S 1}\right), & \text { if } i>i_{Q_{s}^{S 1}}\end{cases}
$$

with $\Pi_{s}^{S 1}\left(Q_{s}^{S 1}, i ; Q_{r}^{S 1} \mid i\right)=w Q_{r}^{S 1} \mid i+\left[v_{s}+\rho\left(c-v_{s}\right)\right]\left(Q_{s}^{S 1}-Q_{r}^{S 1} \mid i\right)$ and $\Pi_{s}^{S 1}\left(Q_{s}^{S 1}, i ; Q_{s}^{S 1}\right)=w Q_{s}^{S 1}$.

Then, at the beginning of Stage 1, the supplier's optimal ordering quantity $Q_{s}^{S 1^{*}}$ of ORS-1 is obtained by solving the optimization problem (P6).

$$
\begin{array}{ll}
\underset{Q_{s}^{S 1}}{\operatorname{maximize}} & \Pi_{s}^{S 1}\left(Q_{s}^{S 1}\right) \\
\text { subject to } & Q_{s}^{S 1}>0,
\end{array}
$$

where $\Pi_{s}^{S 1}\left(Q_{s}^{S 1}\right)=\int_{0}^{i} Q_{s}^{S 1} \Pi_{s}^{S 1}\left(Q_{s}^{S 1}, i ; Q_{r}^{S 1} \mid i\right) \mathrm{d} G(I)+\int_{i_{Q_{s}^{S 1}}}^{+\infty} \Pi_{s}^{S 1}\left(Q_{s}^{S 1}, i ; Q_{s}^{S 1}\right) \mathrm{d} G(I)-c Q_{s}^{S 1}$. Similarly, we obtain the supplier's optimal production quantity $Q_{s}^{S 1^{*}}$ of ORS-1 model in following proposition.

Proposition 4.9. In stage $1, \Pi_{s}^{S 1}\left(Q_{s}^{S 1}\right)$ is concave in $Q_{s}^{S 1}$, and $Q_{s}^{S 1^{*}}$ satisfies $G\left(i_{Q_{s}^{S 1^{*}}}\right)=\frac{w-c}{w-v_{s}-\rho\left(c-v_{s}\right)}$. In addition, when market demand follows the uniform-uniform distribution, $Q_{s}^{S 1^{*}}=\gamma+\frac{\alpha-\beta}{2}+\frac{p-w+\rho\left(c-v_{s}\right)}{p-v_{r}} \beta-$ $\frac{(1-r h o)\left(c-v_{s}\right)}{w-v_{s}-\rho\left(c-v_{s}\right)} \alpha$.

Proposition 4.9 describes the supplier's optimal ordering quantity in the ORS-1 model. Clearly, $Q_{r}^{S 1^{*}}$ increases in $\rho$. The higher sharing proportion of overproduction is, the more the supplier produces. It is because that part of the risk of overproduction for the supplier is carried by the retailer, which gives the supplier an incentive to produce more products. The overproduction-risk sharing mechanism can be view as a transfer payment from the retailer to the supplier. It is worth mentioning that the ORS-1 model is applied when the supplier has dominant power in the supply chain. 


\subsubsection{Overstock risk-sharing model (ORS-2)}

In the ORS-2 model, the overstock risk is shared between the supplier and the retailer, while the overproduction risk is carried only by the supplier. In this setting, at the beginning of Stage 1, the supplier produces $Q_{s}^{S 2}$. At the beginning of Stage 2, the retailer orders $Q_{r}^{S 2}$ with a more accurate latest demand information. If there is any leftover at the end of Stage 2, then the supplier would pay $\phi\left(w-v_{r}\right)\left(Q_{r}^{S 2}-x\right)^{+}$to the retailer to share part of the overstock risk. Under ORS-2 model, the retailer's problem in Stage 2 is following optimization problem.

Similarly, given $Q_{s}^{S 2}$ and $I=i$ at the beginning of Stage 2, the retailer's optimal ordering quantity $Q_{r}^{S 2^{*}}$ is obtained by solving the optimization problem (P7).

$$
\begin{array}{ll}
\underset{Q_{r}^{S 2}}{\operatorname{maximize}} & \Pi_{r}^{S 2}\left(Q_{s}^{S 2}, i ; Q_{r}^{S 2}\right) \\
\text { subject to } & Q_{r}^{S 2} \leq Q_{s}^{S 2},
\end{array}
$$

where $\Pi_{r}^{S 2}\left(Q_{s}^{S 2}, i ; Q_{r}^{S 2}\right)=E_{\{x \mid i\}}\left\{p \min \left(x, Q_{r}^{S 2}\right)-w Q_{r}^{S 2}+\left[v_{r}+\phi\left(w-v_{r}\right)\right]\left(Q_{r}^{S 2}-x\right)^{+}\right\}$. Considering $Q_{r}^{S 2} \leq Q_{s}^{S 2}$, let $Q_{r}^{S 2} \mid i=F^{-1}\left(\frac{p-w}{p-v_{r}-\phi\left(w-v_{r}\right)}\right)$, then the following proposition gives the retailer's optimal order quantity $Q_{r}^{S 2^{*}}$ of the ORS-2 model.

Proposition 4.10. Given $Q_{s}^{S 2}$ and $I=i$ in Stage $2, \Pi_{r}^{S 2}\left(Q_{s}^{S 2}, i ; Q_{r}^{S 2}\right)$ is concave in $Q_{r}^{S 2}$, and

$$
Q_{r}^{S 2^{*}}= \begin{cases}Q_{r}^{S 2} \mid i, & \text { if } i \leq i_{Q_{s}^{S 2}}, \\ Q_{s}^{S 2}, & \text { if } i>i_{Q_{s}^{S 2}}\end{cases}
$$

where $i_{Q_{s}^{S 2}}$ satisfies $Q_{r}^{S 2} \mid i_{Q_{s}^{S 2}}=Q_{s}^{S 2}$.

If market demand is the uniform-uniform distributed, Proposition 4.10 becomes as follows.

Corollary 4.11. Given $Q_{s}^{S 2}$ and $I=i$ in Stage 2, there is a positive threshold $i_{Q_{s}^{S 2}}=Q_{s}^{S 2}+\frac{\beta}{2}-\frac{p-w}{p-v_{r}-\phi\left(w-v_{r}\right)} \beta$ such that

$$
Q_{r}^{S 2^{*}}= \begin{cases}Q_{r}^{S 2} \mid i=i-\frac{\beta}{2}+\frac{p-w}{p-v_{r}-\phi\left(w-v_{r}\right)} \beta, & \text { if } \gamma-\frac{\alpha}{2} \leq i \leq i_{Q_{s}^{S 2}}, \\ Q_{s}^{S 2}, & \text { if } i_{Q_{s}^{S 2}}<i \leq \gamma+\frac{\alpha}{2} .\end{cases}
$$

Proposition 4.10 shows that the retailer's optimal ordering quantity in the ORS-2 model is influenced by the supplier's production quantity, updated demand information and sharing proportion of overstock. Specially, $Q_{r}^{S 2^{*}}$ increases in $\phi$. That is, the bigger the sharing proportion of overstock is, the more the retailer would order. Since part of the risk of overstock for the retailer is carried by the supplier, which gives the retailer an incentive to order more products. From Proposition 4.10, the supplier's optimal expected profit of ORS-2 model in Stage 2 is

$$
\Pi_{s}^{S 2}\left(Q_{s}^{S 2}, i ; Q_{r}^{S 2^{*}}\right)= \begin{cases}\Pi_{s}^{S 2}\left(Q_{s}^{S 2}, i ; Q_{r}^{S 2} \mid i\right), & \text { if } i \leq i_{Q_{s}^{S 2}}, \\ \Pi_{s}^{S 2}\left(Q_{s}^{S 2}, i ; Q_{s}^{S 2}\right), & \text { if } i>i_{Q_{s}^{S 2}}\end{cases}
$$

with $\Pi_{s}^{S 2}\left(Q_{s}^{S 2}, i ; Q_{r}^{S 2} \mid i\right)=w Q_{r}^{S 2} \mid i+v_{s}\left(Q_{s}^{S 2}-Q_{r}^{S 2} \mid i\right)-\phi\left(w-v_{r}\right)\left(Q_{s}^{S 2} \mid i-Q_{r}^{S 2}\right)$ and $\Pi_{s}^{S 2}\left(Q_{s}^{S 2}, i ; Q_{s}^{S 2}\right)=$ $w Q_{s}^{S 2}-\phi\left(w-v_{r}\right)\left(Q_{s}^{S 2}-x\right)^{+}$.

Then, at the beginning of Stage 1, the supplier's optimal ordering quantity $Q_{s}^{S 2^{*}}$ of ORS-2 model is obtained by solving the optimization problem (P8).

$$
\begin{array}{ll}
\underset{Q_{s}^{S 2}}{\operatorname{maximize}} & \Pi_{s}^{S 2}\left(Q_{s}^{S 2}\right) \\
\text { subject to } & Q_{s}^{S 2}>0,
\end{array}
$$

where $\Pi_{s}^{S 2}\left(Q_{s}^{S 2}\right)=\int_{0}^{i_{Q s}^{S 2}} \Pi_{s}^{S 2}\left(Q_{s}^{S 2}, i ; Q_{r}^{S 2} \mid i\right) \mathrm{d} G(I)+\int_{i_{Q_{s}^{S 2}}}^{+\infty} \Pi_{s}^{S 2}\left(Q_{s}^{S 2}, i ; Q_{s}^{S 2}\right) \mathrm{d} G(I)-c Q_{s}^{S 2}$. The supplier's optimal production quantity $Q_{s}^{S 2^{*}}$ of ORS-2 model is given in following proposition. 
Proposition 4.12. In stage $1, \Pi_{s}^{S 2}\left(Q_{s}^{S 2}\right)$ is concave in $Q_{s}^{S 2}$, and $Q_{s}^{S 2^{*}}$ is the unique solution of the following equation:

$$
\int_{i_{Q_{s}^{S 2}}}^{+\infty}\left[\frac{w-v_{s}}{\phi\left(w-v_{r}\right)}-F\left(Q_{s}^{S 2} \mid i\right)\right] \mathrm{d} G(I)=\frac{c-v_{s}}{\phi\left(w-v_{r}\right)} .
$$

In addition, when market demand follows the uniform-uniform distribution, $Q_{s}^{S 2^{*}}=\gamma+\frac{\alpha-\beta}{2}+\frac{w-v_{s}}{\phi\left(w-v_{r}\right)} \beta-$ $\sqrt{\left[\frac{w-v_{s}}{\phi\left(w-v_{r}\right)} \beta-\frac{p-w}{p-v_{r}-\phi\left(w-v_{r}\right)} \beta\right]^{2}+\frac{2\left(c-v_{s}\right) \alpha \beta}{\phi\left(w-v_{r}\right)}}$.

Proposition 4.12 characterizes the sufficient condition of the optimal production quantity in the ORS-2 model. The overstock-risk sharing mechanism is view as a transfer payment from the supplier to the retailer. To make production plan in this model, the supplier has to evaluate the trade-off between a possible higher ordering at the beginning of Stage 2 and a potentially higher extra cost burden at the end of Stage 2 . It is worth mentioning that the ORS-2 model is applied when the retailer has dominant power in the supply chain.

\subsubsection{Bi-directional risk-sharing model (BRS)}

In the BRS model, both the overproduction risk and the overstock risk are shared between the supplier and the retailer. In this setting, at the beginning of Stage 1, the supplier's production quantity is $Q_{s}^{B}$. At the beginning of Stage 2, the retailer's order quantity is $Q_{r}^{B}$. In the case of $Q_{r}^{B}<Q_{s}^{B}$, the retailer would pay $\rho\left(c-v_{s}\right)\left(Q_{s}^{B}-Q_{r}^{B}\right)$ to the supplier at the end of Stage 1. In the case of $Q_{r}^{B}>x$, the supplier would pay $\phi(w-r)\left(Q_{r}^{B}-x\right)^{+}$to the retailer at the end of Stage 2. Next, we further solve this two-stage optimization problem by using a backward induction method.

Given $Q_{s}^{B}$ and $I=i$ at the beginning of Stage 2, we obtain the retailer's optimal ordering quantity $Q_{r}^{B^{*}}$ of BRS model by solving the optimization problem (P9).

$$
\begin{array}{ll}
\underset{Q_{r}^{B}}{\operatorname{maximize}} & \Pi_{r}^{B}\left(Q_{s}^{B}, i ; Q_{r}^{B}\right) \\
\text { subject to } & Q_{r}^{B} \leq Q_{s}^{B},
\end{array}
$$

where $\Pi_{r}^{B}\left(Q_{s}^{B}, i ; Q_{r}^{B}\right)=E_{\{x \mid i\}}\left\{p \min x, Q_{r}^{B}-w Q_{r}^{B}+\left[\phi\left(w-v_{r}\right)+v_{r}\right]\left(Q_{r}^{B}-x\right)^{+}-\rho\left(c-v_{s}\right)\left(Q_{s}^{B}-Q_{r}^{B}\right)\right\}$. The retailer's goal is to order a feasible quantity to maximize $\Pi_{r}^{B}\left(Q_{s}^{B}, i ; Q_{r}^{B}\right)$ subjected to $Q_{r}^{B} \leq Q_{s}^{B}$. Let $Q_{r}^{B} \mid i=F^{-1}\left(\frac{p-w+\rho\left(c-v_{s}\right)}{p-v_{r}-\phi\left(w-v_{r}\right)} \mid i\right)$, we have following proposition.

Proposition 4.13. Given $Q_{s}^{B}$ and $I=i$ in Stage $2, \Pi_{r}^{B}\left(Q_{s}^{B}, i ; Q_{r}^{B}\right)$ is concave in $Q_{r}^{B}$, and

$$
Q_{r}^{B^{*}}= \begin{cases}Q_{r}^{B} \mid i, & \text { if } i \leq i_{Q_{s}^{B}}, \\ Q_{s}^{B}, & \text { if } i>i_{Q_{s}^{B}},\end{cases}
$$

where $i_{Q_{s}^{B}}$ satisfies $Q_{r}^{B} \mid i_{Q_{s}^{B}}=Q_{s}^{B}$.

When market demand is the uniform-uniform distributed, Proposition 4.13 becomes following corollary.

Corollary 4.14. Given $Q_{s}^{B}$ and $I=i$ in Stage 2, there is a positive threshold $i_{Q_{s}^{B}}=Q_{s}^{B}+\frac{\beta}{2}-\frac{p-w+\rho\left(c-v_{s}\right)}{p-v_{r}-\phi\left(w-v_{r}\right)} \beta$ such that

$$
Q_{r}^{B^{*}}= \begin{cases}Q_{r}^{B} \mid i=i-\frac{\beta}{2}+\frac{p-w+\rho\left(c-v_{s}\right)}{p-v_{r}-\phi\left(w-v_{r}\right)} \beta, & \text { if } \gamma-\frac{\alpha}{2} \leq i \leq i_{Q_{s}^{B}}, \\ Q_{s}^{B}, & \text { if } i_{Q_{s}^{B}}<i \leq \gamma+\frac{\alpha}{2} .\end{cases}
$$

Proposition 4.13 shows that, for any given the supplier's production quantity and updated demand information in Stage 2, the retailer's optimal ordering quantity of BRS model is influenced by both sharing proportions of overproduction and overstock. Specifically, given $w$ and $\rho, Q_{r}^{B} \mid i$ is increasing in $\phi$. It indicates that the retailer 
orders more if the supplier shares greater overstock risk. Given $w$ and $\phi, Q_{r}^{B} \mid i$ is also increasing in $\rho$. For any a given $Q_{s}^{B}$, the amount that the retailer shares the supplier's overproduction risk is $\rho\left(c-v_{s}\right)\left(Q_{s}^{B}-Q_{r}^{B} \mid i\right)$, which increases with $\rho$. Comparatively, the amount that the retailer obtains the supplier's compensation for overstock risk is $\phi\left(w-v_{r}\right)\left(Q_{r}^{B} \mid i-x\right)$, which increases with $Q_{r}^{B} \mid i$. It means that ordering more makes the retailer obtain greater compensation. As $\rho$ increases, the retailer would order more quantity so that she enjoys greater compensation to balance payment. Furthermore, from Proposition 4.13, the supplier's optimal expected profit of BRS model in Stage 2 is

$$
\Pi^{B}\left(Q_{s}^{B}, i ; Q_{r}^{B^{*}}\right)= \begin{cases}\Pi^{B}\left(Q_{s}^{B}, i ; Q_{r}^{B} \mid i\right), & \text { if } i \leq i_{Q_{s}^{B}}, \\ \Pi^{B}\left(Q_{s}^{B}, i ; Q_{s}^{B}\right), & \text { if } i>i_{Q_{s}^{B}},\end{cases}
$$

where

$$
\Pi^{B}\left(Q_{s}^{B}, i ; Q_{r}^{B} \mid i\right)=w Q_{r}^{B} \mid i+\left[v_{s}+\rho\left(c-v_{s}\right)\right]\left(Q_{s}^{B}-Q_{r}^{B} \mid i\right)-\phi\left(w-v_{r}\right)\left(Q_{r}^{B} \mid i-x\right)^{+},
$$

and

$$
\Pi^{B}\left(Q_{s}^{B}, i ; Q_{s}^{B}\right)=w Q_{s}^{B}-\phi\left(w-v_{r}\right)\left(Q_{s}^{B}-x\right)^{+} .
$$

Finally, at the beginning of Stage 1, the supplier's optimal ordering quantity $Q_{s}^{B^{*}}$ of BRS model is given in following the optimization problem (P10).

$$
\begin{array}{cc}
\underset{Q_{s}^{B}}{\operatorname{maximize}} & \Pi_{s}^{B}\left(Q_{s}^{B}\right) \\
\text { subject to } & Q_{s}^{B}>0,
\end{array}
$$

where $\Pi_{s}^{B}\left(Q_{s}^{B}\right)=\int_{0}^{i_{Q_{s}^{B}}} \Pi_{s}^{B}\left(Q_{s}^{B}, i ; Q_{r}^{B} \mid i\right) \mathrm{d} G(I)+\int_{i_{Q_{s}^{B}}^{+\infty}}^{+\infty} \Pi_{s}^{B}\left(Q_{s}^{B}, i ; Q_{s}^{B}\right) \mathrm{d} G(I)-c Q_{s}^{B}$. We have the following proposition to describe the optimal production quantity of the supplier $Q_{s}^{B^{*}}$ in the BRS model.

Proposition 4.15. In stage $1, \Pi_{s}^{B}\left(Q_{s}^{B}\right)$ is concave in $Q_{s}^{B}$, and $Q_{s}^{B^{*}}$ is the unique solution of the following equation:

$$
\int_{i_{Q_{s}^{B}}}^{+\infty}\left[\frac{w-v_{s}-\rho\left(c-v_{s}\right)}{\phi\left(w-v_{r}\right)}-F\left(Q_{s}^{B} \mid i\right)\right] \mathrm{d} G(I)=\frac{(1-\rho)\left(c-v_{s}\right)}{\phi\left(w-v_{r}\right)} .
$$

In addition, when market demand follows the uniform-uniform distribution, $Q_{s}^{B^{*}}=\gamma+\frac{\alpha-\beta}{2}+\frac{w-v_{s}-\rho\left(c-v_{s}\right)}{\phi\left(w-v_{r}\right)} \beta-$ $\sqrt{\left[\frac{w-v_{s}-\rho\left(c-v_{s}\right)}{p-v_{r}-\phi\left(w-v_{r}\right)} \beta-\frac{p-w+\rho\left(c-v_{s}\right)}{p-v_{r}-\phi\left(w-v_{r}\right)} \beta\right]^{2}+\frac{2(1-\rho)\left(c-v_{s}\right) \alpha \beta}{\phi\left(w-v_{r}\right)}}$.

Proposition 4.15 characterizes the sufficient condition of the supplier's optimal production quantity in the BRS model. From the right-hand side of equation (4.7), it can be observed that the optimal production quantity $Q_{s}^{B^{*}}$ is influenced by the product of the cost-wholesale price ratio (i.e., $\frac{c-v_{s}}{w-v_{r}}$ and the sharing proportions ratio (i.e., $\frac{1-\rho}{\phi}$ ). In practice, there are many various factors which influence the parameter settings. For example, in Section 6 , we will discuss how to set contract terms $\{w, \rho, \phi\}$ according to supply chain members' risk preferences and negotiating powers.

\subsection{Model comparison and analysis}

In this subsection, we further compare the supplier's optimal production decision and the retailer's optimal ordering decision in different models, and analysis how the various risk-sharing mechanisms affect the supply chain members' quantity decisions. 
Lemma 4.16. $Q_{s}^{S 1^{*}}>Q_{s}^{N^{*}}$.

Lemma 4.16 shows that the supplier would produce more in the ORS-1 model than the NRS model. This makes sense because the supplier has to carry all overproduction risk in the NRS model. However, in the ORS-1 model, the supplier carries less pressure and has an incentive to produce more. Next, we study the difference of the retailer's optimal order quantity $Q_{r}^{j^{*}}$ in these models. Note that $Q_{r}^{j^{*}}$ has two possible scenarios, which depends on the values of $i_{Q_{s}^{j^{*}}}$. We first consider the case of $i<i_{Q_{s}^{j^{*}}}$, i.e., $Q_{r}^{j^{*}}=Q_{r}^{j^{*}} \mid i$, then we have the following lemma.

Lemma 4.17. (1) $Q_{r}^{B^{*}}\left|i>Q_{r}^{S 1^{*}}\right| i>Q_{r}^{N^{*}}\left|i ;(2) \quad Q_{r}^{B^{*}}\right| i>Q_{r}^{S 2^{*}}\left|i>Q_{r}^{N^{*}}\right| i$.

From Lemma 4.17, when $i<i_{Q_{s}^{j^{*}}}$, we can see that: (1) the retailer orders more from the supplier in the models of ORS-1, ORS-2 and BRS than in the model of NRS. It indicates that three risk-sharing mechanisms can give the retailer an incentive to order more. (2) Compared to a single risk-sharing mechanism (e.g., ORS-1 and ORS-2), a bi-directional risk-sharing mechanism provides more incentive for the retailer to order. Next, we consider the case of $i \geq i_{Q_{s}^{j^{*}}}$, and give the following lemma which directly from Lemmas 4.16 and 4.17.

Lemma 4.18. $Q_{r}^{S 1^{*}}>Q_{r}^{N^{*}}$.

Lemma 4.18 shows that the retailer always orders more in the ORS- 1 model than in the NRS model. It is quite surprising that sharing the risk of overproduction for the supplier would incent the retailer to order more. However, the relationship between $Q_{s}^{S 1^{*}}$ and $Q_{s}^{S 2^{*}}$ is not clear. Parameters in the four risk-sharing models are very complicated and it's difficult to acquire clear analytical results. Therefore, we will use some numerical examples in Section 7.

\section{Supply CHAin COORDinAtion AND PERFORMANCE EVALUATION}

In this section, we study the contract of supply chain coordination with demand information updating from risk-sharing perspective. For convenience, we summarize the optimal quantity decisions of centralized and decentralized systems in Table 3.

Table 3 shows that $Q_{r}^{c}\left|i>Q_{r}^{j}\right| i$ since $w>c>v_{S}>v_{r}$ with $j=N, S 1, S 2, B$. It shows that that the supply chain members' quantity decisions in the decentralized system are difficult to reach the centralized system optimal level. Spengler [35] attributes this to "double marginalization", which would lead a poor supply chain performance. It can be noted from Table 3 that both the supplier's wholesale price and the sharing proportions of overproduction and overstock have a direct impact on the supplier's production decision and the retailer's ordering decision. Therefore, the supply chain members can negotiate to adjust the contract parameters $\{w, \rho, \phi\}$ in different models so that the both members are willing to adopt the quantity policy that optimizes the system. To achieve such coordination, we first let $Q_{r}^{\mathrm{SC}}=Q_{r}^{j^{*}}$, i.e., the retailer's optimal order decision in the decentralized system equals to its counterpart in the centralized system. According to the discussion in Section 4.2, there exists two possible situations for the retailer's ordering decision. When the supplier's production quantity in the decentralized system is enough to satisfy the optimal order quantity in the centralized system (i.e., $Q_{s}^{\mathrm{SC}^{*}} \geq Q_{r}^{j^{*}}$ ), then the sufficient condition to coordinate the supply chain can be derived from $F\left(Q_{r}^{\mathrm{SC}^{*}} \mid i\right)=F\left(Q_{r}^{j^{*}} \mid i\right)$. When the production quantity is not enough to satisfy the optimal order quantity in the centralized system (i.e., $Q_{s}^{\mathrm{SC}^{*}}<Q_{r}^{j^{*}}$ ), the retailer can only order the fresh product up to the initial production quantity, i.e., $Q_{s}^{j^{*}}=Q_{r}^{\mathrm{SC}^{*}}$.

In what follows, taking the BRS model as an example, we derive the sufficient conditions whereby the bidirectional risk-sharing contract coordinates the supply chain with demand information updating. To be more specific, let $F\left(Q_{r}^{\mathrm{SC}^{*}} \mid i\right)=F\left(Q_{r}^{B^{*}} \mid i\right)$, i.e., the retailer's optimal order quantity in the decentralized system equals the to its counterpart in the centralized system. Then, we obtain

$$
w=p+\rho\left(c-v_{s}\right)-\frac{p-v_{s}}{p-v_{r}}\left[p-v_{r}-\phi\left(w-v_{r}\right)\right] .
$$


TABLE 3. Optimal quantity decisions with demand information updating.

\begin{tabular}{|c|c|c|}
\hline Model & Ordering quantity in Stage 2 & Production quantity in Stage 1 \\
\hline Centralized & $\begin{array}{l}Q_{r}^{\mathrm{SC}^{*}}=Q_{r}^{\mathrm{SC}} \mid i, \text { if } i \leq i_{Q_{s}^{\mathrm{SC}^{*}}}, \\
Q_{s}^{\mathrm{SC}^{*}}=Q_{s}^{\mathrm{SC}}, \quad \text { if } i>i_{Q_{s}^{\mathrm{SC}^{*}}}, \\
\text { where } F\left(Q_{r}^{\mathrm{SC}^{*}} \mid i\right)=\frac{p-v_{s}}{p-v_{r}}\end{array}$ & 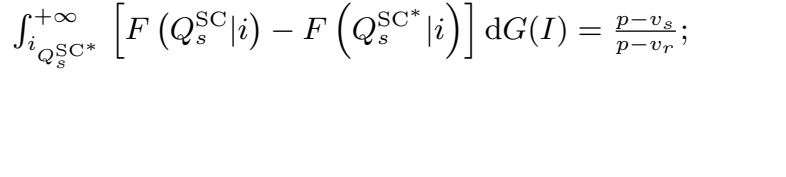 \\
\hline NRS & $\begin{array}{l}Q_{r}^{N^{*}}=Q_{r}^{N} \mid i, \quad \text { if } i \leq i_{Q_{s}^{N^{*}}}, \\
Q_{s}^{N^{*}}=Q_{s}^{N}, \quad \text { if } i>i_{Q_{s}^{N^{*}}}, \\
\text { where } F\left(Q_{r}^{N^{*}} \mid i\right)=\frac{p-w}{p-v_{r}}\end{array}$ & $G\left(i_{Q_{s}^{N *}}\right)=\frac{w-c}{w-v_{s}}$ \\
\hline ORS-1 & $\begin{array}{l}Q_{r}^{S 1^{*}}=Q_{r}^{S 1} \mid i, \quad \text { if } i \leq i_{Q_{s}^{S 1^{*}}} \\
Q_{s}^{S 1^{*}}=Q_{s}^{S 1}, \quad \text { if } i>i_{Q_{s}^{S 1^{*}}} \\
\text { where } F\left(Q_{r}^{S 1^{*}} \mid i\right)=\frac{p-w+\rho\left(c-v_{s}\right)}{p-v_{r}}\end{array}$ & $G\left(i_{Q S 1^{*}}\right)=\frac{w-c}{w-v_{s}-\rho\left(c-v_{s}\right)}$ \\
\hline ORS-2 & $\begin{array}{l}Q_{r}^{S 2^{*}}=Q_{r}^{S C} \mid i, \text { if } i \leq i_{Q_{s}^{S 2^{*}}} \\
Q_{s}^{S 2^{*}}=Q_{s}^{S 2}, \quad \text { if } i>i_{Q_{s}^{S 2^{*}}} \\
\text { where } F\left(Q_{r}^{S 2^{*}} \mid i\right)=\frac{p-w}{p-v_{r}-\phi\left(w-v_{r}\right)}\end{array}$ & $\int_{i_{Q_{s}^{S 2^{*}}}^{+\infty}}^{+\infty}\left[\frac{w-v_{s}}{\phi\left(w-v_{r}\right)}-F\left(Q_{s}^{S 2^{*}} \mid i\right)\right] \mathrm{d} G(I)=\frac{c-v_{s}}{\phi\left(w-v_{r}\right.}$ \\
\hline BRS & $\begin{array}{l}Q_{r}^{B^{*}}=Q_{r}^{B} \mid i, \quad \text { if } i \leq i_{Q_{s}^{B^{*}}} \\
Q_{s}^{B^{*}}=Q_{s}^{B}, \quad \text { if } i>i_{Q_{B}^{B^{*}}} \\
\text { where } F\left(Q_{r}^{B^{*}} \mid i\right)=\frac{p-w+\rho\left(c-v_{s}\right)}{p-v_{r}-\phi\left(w-v_{r}\right)}\end{array}$ & $\int_{i_{Q_{s}^{B *}}^{+\infty}}^{+\infty}\left[\frac{w-v_{s}-\rho\left(c-v_{s}\right)}{\phi\left(w-v_{r}\right)}-F\left(Q_{s}^{B^{*}} \mid i\right)\right] \mathrm{d} G(I)=\frac{(1-\rho)\left(c-v_{s}\right)}{\phi\left(w-v_{r}\right.}$ \\
\hline
\end{tabular}

Inserting this w into $F\left(Q_{r}^{B^{*}} \mid i\right)=\frac{p-w+\rho\left(c-v_{s}\right)}{p-v_{r}-\phi\left(w-v_{r}\right)}$ and $(4.7)$, then we have $F\left(Q_{r}^{B^{*}} \mid i\right)=\frac{p-v_{s}}{p-v_{r}}=F\left(Q_{r}^{\mathrm{SC}} \mid i\right)$ and

$$
\int_{i_{Q_{s}^{B}}}^{+\infty}\left[F\left(Q_{r}^{\mathrm{SC}^{*}} \mid i\right)-F\left(Q_{s}^{B^{*}} \mid i\right)\right] \mathrm{d} G(i)=\frac{1-\rho\left(c-v_{s}\right)}{\phi\left(w-v_{r}\right)} .
$$

Compared above equation with (4.4), as long as

$$
\frac{(1-\rho)\left(c-v_{s}\right)}{\phi\left(w-v_{r}\right)}=\frac{c-v_{s}}{p-v_{r}}
$$

i.e.,

$$
\rho=1-\frac{w-v_{r}}{p-v_{r}} \phi
$$

then, equations (4.4) and (4.7) are equivalent, which indicates that the supplier's optimal production quantity in the decentralized system equals its counterpart in the centralized system. Similar operations are performed in other risk-sharing models. Thus, we characterize the sufficient conditions of the supply chain coordination under different risk-sharing contracts in the following proposition.

Proposition 5.1. The supply chain can be coordinate only when

(1) $w=c$ in the NRS model;

(2) $w=c=v_{s}$ in the ORS-1 model;

(3) $w=c=v_{s}=v_{r}$ in the ORS-2 model; 
(4) $w=p-\rho(p-c), \quad(1-\rho)\left(p-v_{r}\right)=\phi\left(w-v_{r}\right)$ in the BRS model.

In addition, when the supply chain coordination is achieved in the models of NRS, ORS-1 and ORS-2, the supplier makes zero profits.

From Proposition 5.1(1)-(3), we see that $w=c$ is a necessary condition for supply chain coordination in the models of NRS, ORS-1 and ORS-2. It indicates that the supply chain in the models of NRS, ORS-1 and ORS-2 can be coordinated only if the supplier earns a zero profit. This means that the supply chain of NRS, ORS-1 and ORS-2 models can achieve coordination may not be individually rational for the supplier. That is, he may be unwilling to participate to achieve coordination. We further verify whether the parameter setting in Proposition 5.1(4) can induce the supplier to participate voluntarily in coordinating supply chain in the BRS model. We first check the supplier's production decision at the beginning of Stage 2. Substituting the parameter setting of Proposition 5.1(4) into equations (4.5) and (4.6) yields the supplier's optimal expected profit of BRS model in Stage 2:

$$
\Pi_{s}^{B}\left(Q_{s}^{B}, i ; Q_{r}^{B} \mid i\right)=(1-\rho)\left[\left(p-v_{s}\right) Q_{r}^{B} \mid i-\left(p-v_{r}\right)\left(Q_{r}^{B} \mid i-x\right)^{+}+v_{s} Q_{s}^{B}\right]+\rho c Q_{s}^{B},
$$

and

$$
\Pi_{s}^{B}\left(Q_{s}^{B}, i ; Q_{s}^{B}\right)=(1-\rho)\left[p Q_{s}^{B}-\left(p-v_{r}\right)\left(Q_{s}^{B}-x\right)^{+}\right]+\rho c Q_{s}^{B} .
$$

Comparing equations (5.1), (5.2) with equations (4.2), (4.3), it becomes clear that the supplier's expected profit in Stage 2 coincides with that in the centralized case. In Stage 1, only the supplier makes the production quantity decision. Combining equations (5.1), (5.2) and taking expectation over $I=i$, it is easy to see that the supplier's profit function coincides with $\Pi^{\mathrm{SC}}\left(Q_{s}^{\mathrm{SC}}, i ; Q_{r}^{\mathrm{SC}}\right)$. Therefore, in the BRS model, the supply chain is coordinated with parameter setting of Proposition 5.1(4). In particular, we establish following proposition to demonstrate that the bi-directional risk-sharing contract $\{w, \rho, \phi\}$ permits an arbitrary allocation of the supply chain profit between the supplier and the retailer.

Proposition 5.2. With $\{w, \rho, \phi\} \in M$, the optimal expected profit of the centralized SC with demand information updating can be reached, where

$$
M=\left\{\{w, \rho, \phi\}: w=p-\rho(p-c),(1-\rho)\left(p-v_{r}\right)=\phi\left(w-v_{r}\right), \rho \in[0,1]\right\} .
$$

Furthermore, $\Pi_{r}^{B}\left(Q_{s}^{B}\right)=\rho \Pi^{\mathrm{SC}}\left(Q_{s}^{\mathrm{SC}}\right)$ and $\Pi_{r}^{B}\left(Q_{s}^{B}\right)=(1-\rho) \Pi^{\mathrm{SC}}\left(Q_{s}^{\mathrm{SC}}\right)$.

From Proposition 5.2, through setting a feasible wholesale price to realize $Q_{r}^{B}\left|i=Q_{r}^{\mathrm{SC}}\right| i$ with $\{w, \rho, \phi\} \in M$, the optimal expected profit of the centralized system is reached. Note that $w$ has a negative correlation with $\rho$. The supplier is willing to charge a lower wholesale price if the retailer commits a higher sharing proportion of overproduction. Meanwhile, increasing $\rho$ leads to $\phi$ decreasing. That is, even if the retailer shares a higher overproduction proportion, the supplier cannot increase overstock sharing proportion. Hence, the parameter of $\rho$ plays a key role in splitting the supply-chain profit between the supplier and the retailer. The higher the risk that the retailer faces, the more profit she is expected to acquire, and likewise for the supplier. The negotiation between the supply chain members can then focus on the risk that each member is able and willing to take. Therefore, the bi-directional risk-sharing contract can be accepted voluntarily by supply chain members.

Furthermore, from Proposition 5.2, one can derive that as $\rho$ increases the retailer's expected profit increases while the supplier's expected profit decreases. Hence, the parameter $\rho$ plays a key role in splitting the supply chain's profit between the two members. Following Zhao et al. [49], to evaluate the performance of the bidirectional risk-sharing contract comparing with single risk-sharing contract (i.e., NRS, ORS-1 and ORS-2 models), we define the profit gaps between $\{w, \rho, \phi\} \in M$ and $\{w\},\{w, \rho\},\{w, \phi\}$ as

$$
\operatorname{Gap}_{s}^{k}=\frac{\Pi_{s}^{B}\left(Q_{s}^{B^{*}}\right)-\Pi_{s}^{k}\left(Q_{s}^{k *}\right)}{\Pi_{s}^{B}\left(Q_{s}^{B *}\right)} \text { and } \operatorname{Gap}_{r}^{k}=\frac{\Pi_{r}^{B}\left(Q_{r}^{B^{*}}\right)-\Pi_{r}^{k}\left(Q_{r}^{k *}\right)}{\Pi_{r}^{B}\left(Q_{r}^{B^{*}}\right)} \text {, with } k=N, S 1, S 2 \text {, }
$$


where $\operatorname{Gap}_{s}^{k}$ and $\mathrm{Gap}_{r}^{k}$ represent the supplier's and the retailer's profit advantage with $\{w, \rho, \phi\} \in M$ over $\{w\}$, $\{w, \rho\}$ and $\{w, \phi\}$, respectively.

Both supply chain members would accept $\{w, \rho, \phi\} \in M$ only when $\operatorname{Gap}_{s}^{k} \geq 0$ and $\operatorname{Gap}_{r}^{k} \geq 0$. Then, compared with $\{w\},\{w, \rho\}$ and $\{w, \phi\}$, the following proposition establishes the conditions in which the supply chain coordination is achieved under $\{w, \rho, \phi\} \in M$.

Proposition 5.3. Compared with $\{w\},\{w, \rho\}$ and $\{w, \phi\}$, the coordination of the supply chain is achieved under $\{w, \rho, \phi\} \in N \in M$, where $N=\left\{\{w, \rho, \phi\}: w=p-\rho(p-c),(1-\rho)\left(p-v_{r}\right)=\phi\left(w-v_{r}\right), \rho \in\left[\rho_{\min }^{k}, \rho_{\max }^{k}\right]\right\}$, where $\rho_{\min }^{k}=\frac{\Pi_{r}^{k}\left(Q_{r}^{k *}\right)}{\Pi^{\mathrm{SC}}\left(Q_{s}^{\mathrm{SC}}\right)}$ and $\rho_{\max }^{k}=1-\frac{\Pi_{s}^{k}\left(Q_{s}^{k *}\right)}{\Pi^{\mathrm{SC}}\left(Q_{s}^{\mathrm{SC}}\right)}$ with $k=N, S 1, S 2$.

Proposition 5.3 shows that with $\{w, \rho, \phi\} \in N \in M$, both the supplier and retailer are beneficial compared with $\{w\},\{w, \rho\}$ and $\{w, \phi\}$. In there, $\operatorname{Gap}_{s}^{k} \geq 0$ and $\operatorname{Gap}_{r}^{k} \geq 0$ with $k=N, S 1, S 2$. To be more specific, when $\rho=\rho_{\min }^{k}$, we have $\Delta \Pi_{r}^{k}=\Pi_{r}^{B}\left(Q_{r}^{B^{*}}\right)-\Pi_{r}^{k}\left(Q_{r}^{k *}\right)=0$, and $\Delta \Pi_{s}^{k}=\Pi_{s}^{B}\left(Q_{s}^{B^{*}}\right)-\Pi_{s}^{k}\left(Q_{s}^{k}\right)=\Pi^{\mathrm{SC}}\left(Q_{c}^{\mathrm{SC}}\right)-\Pi^{k}\left(Q_{r}^{k *}\right):=$ $\Delta \Pi$. The supplier takes all the additional expected profit of the centralized supply chain while the retailer only reserves the initial expected profit of the decentralized supply chain under other models. Comparatively, when $\rho=\rho_{\max }^{k}$, then $\Delta \Pi_{r}^{k}=\Delta \Pi$ and $\Delta \Pi_{s}^{k}=0$. The retailer gains the entire additional expected profit. Through tuning $\rho \in\left[\rho_{\min }^{k}, \rho_{\max }^{k}\right]$, the expected profit of the centralized supply chain is arbitrarily allocated between both members. Compared with $\{w\},\{w, \rho\}$ and $\{w, \phi\}$, the coordination of the supply chain is achieved under $\{w, \rho, \phi\} \in N \in M$.

\section{EFFECTS OF RISK AVERSION AND NEGOTIATION POWER}

In this section, we study how the supply chain members' risk aversion and negotiation power affect the optimal settings of $\{w, \rho, \phi\} \in N$ and the allocation of the additional expected profit of the centralized system. Assume that both members are risk-averse. Following the vN-M utility function [38] and the linear aggregation rule [49], the supply-chain utility function $u\left(\Delta \pi_{r}, \Delta \pi_{s}\right)$ is given by $u\left(\Delta \pi_{r}, \Delta \pi_{s}\right)=\lambda_{r} u_{r}\left(\Delta \pi_{r}\right)+\lambda_{s} u_{s}\left(\Delta \pi_{s}\right)$, where $u_{r}\left(\Delta \pi_{r}\right)$ and $u_{s}\left(\Delta \pi_{s}\right)$ represent the vN-M utility functions of the supplier and retailer respectively when obtaining additional expected profits. The parameters $\lambda_{r}$ and $\lambda_{s}$ are used to measure the negotiation powers of the retailer and supplier respectively, where $\lambda_{r}+\lambda_{s}=1$. A larger value of $\lambda$ denotes a stronger negotiation power of the supply chain member. The optimal contract selection can be obtained by solving the programming problem (P11) as follows.

$$
\begin{array}{cl}
\underset{\rho}{\operatorname{maximize}} & u\left(\Delta \pi_{r}, \Delta \pi_{s}\right) \\
\text { subject to } & \rho \in\left[\rho_{\min }, \rho_{\max }\right],
\end{array}
$$

Following Yan and Wang [45], the utility functions of the supplier and retailer are $u_{r}\left(\Delta \pi_{r}\right)=-\exp ^{-\gamma_{r} \Delta \pi_{r}}$ and $u_{s}\left(\Delta \pi_{s}\right)=-\exp ^{\gamma_{s} \Delta \pi_{s}}$, respectively, where $\gamma_{s}, \gamma_{r}>0$ represent the risk-tolerant coefficients of the supplier and retailer respectively. Based on the Pratt-Arrow risk aversion function [30], we derive the coefficients of the absolute risk aversion of the supplier and retailer as follows.

$$
R_{s}\left(\Delta \pi_{s}\right)=\frac{-u_{s}^{\prime \prime}\left(\Delta \pi_{s}\right)}{u_{s}^{\prime}\left(\Delta \pi_{s}\right)}=\gamma_{s} \text { and } R_{r}\left(\Delta \pi_{r}\right)=\frac{-u_{r}^{\prime \prime}\left(\Delta \pi_{r}\right)}{u_{r}^{\prime}\left(\Delta \pi_{r}\right)}=\gamma_{r}
$$

$R_{s}\left(\Delta \pi_{s}\right)$ and $R_{r}\left(\Delta \pi_{r}\right)$ are strictly increasing in $\gamma_{s}$ and $\gamma_{r}$, respectively. A larger value of $\gamma$ denotes the supply chain member is stronger risk-averse. We present three examples to illustrate how the members' risk aversion and negotiation power affect the selection of optimal contract and the allocation of the additional supply-chain profit.

Example 6.1. We consider a two-echelon supply chain consisting of a risk-averse supplier with the exponential utility function $u_{s}\left(\Delta \pi_{s}\right)=-\exp ^{-\gamma_{s} \Delta \pi_{s}}$ and a risk-averse retailer with the exponential utility function 
$u_{r}\left(\Delta \pi_{r}\right)=-\exp ^{-\gamma_{r} \Delta \pi_{r}}$. Following the linear aggregation rule, the supply chain utility function is

$$
u\left(\Delta \pi_{s}, \Delta \pi_{r}\right)=-\lambda_{r} \exp ^{-\gamma_{r} \Delta \pi_{r}}-\lambda_{s} \exp ^{-\gamma_{s} \Delta \pi_{s}}:=u^{1} .
$$

Solving the program (P11) with $u^{1}$, the following lemma establishes the optimal risk-sharing proportion of the centralized supply chain with both risk-averse members.

Lemma 6.2. When both members are risk-averse,

(i) If $\frac{\lambda_{s}}{\lambda_{r}} \leq \frac{\gamma_{r}}{\gamma_{s}} \exp ^{-\gamma_{r} \Delta \pi}$, then $\rho^{*}=\rho_{\max }, \Delta \pi_{r}=\Delta \pi$ and $\Delta \pi_{s}=0$;

(ii) If $\frac{\gamma_{r}}{\gamma_{s}} \exp ^{-\gamma_{r} \Delta \pi} \leq \frac{\lambda_{s}}{\lambda_{r}} \leq \frac{\gamma_{r}}{\gamma_{s}} \exp ^{\gamma_{s} \Delta \pi}$, then $\rho^{*}=\frac{\gamma_{r}}{\gamma_{r}+\gamma_{s}} \rho_{\min }+\frac{\gamma_{s}}{\gamma_{r}+\gamma_{s}} \rho_{\max }-\frac{\ln \frac{\lambda_{s} \gamma_{s}}{\lambda_{r} \gamma_{r}}}{\left(\gamma_{r}+\gamma_{s}\right) \pi}, \Delta \pi_{r}=\frac{\gamma_{s}}{\gamma_{r}+\gamma_{s}} \Delta \pi-\frac{\ln \frac{\lambda_{s} \gamma_{s}}{\lambda_{r} \gamma_{r}}}{\gamma_{r}+\gamma_{s}}$ and $\Delta \pi_{s}=\frac{\gamma_{r}}{\gamma_{r}+\gamma_{s}} \Delta \pi+\frac{\ln \frac{\lambda_{s} \gamma_{s}}{\lambda_{r} \gamma_{r}}}{\gamma_{r}+\gamma_{s}}$

(iii) If $\frac{\lambda_{s}}{\lambda_{r}} \geq \frac{\gamma_{r}}{\gamma_{s}} \exp ^{\gamma_{s} \Delta \pi}$, then $\rho^{*}=\rho_{\min }, \Delta \pi_{r}=0$ and $\Delta \pi_{s}=\Delta \pi$.

Lemma 6.2 shows that, given $\gamma_{r}$ and $\gamma_{s}$, the optimal risk-sharing proportion of the centralized system with both risk-averse members fully depends on the members' negotiation powers. If the risk-averse supplier's negotiation power relative to the risk-averse retailer is weak (i.e., $\frac{\lambda_{s}}{\lambda_{r}} \leq \frac{\gamma_{r}}{\gamma_{s}} \exp ^{-\gamma_{r} \Delta \pi}$ ), the risk-averse retailer would occupy the entire additional supply chain profit. If the risk-averse supplier's negotiation power relative to the risk-averse retailer's is moderate (i.e., $\frac{\gamma_{r}}{\gamma_{s}} \exp ^{-\gamma_{r} \Delta \pi} \leq \frac{\lambda_{s}}{\lambda_{r}} \leq \frac{\gamma_{r}}{\gamma_{s}} \exp ^{\gamma_{s} \Delta \pi}$ ), there would exist transfer payment between the supplier and retailer. When $\frac{\lambda_{s}}{\lambda_{r}} \geq \frac{\gamma_{r}}{\gamma_{s}}$, the risk-averse retailer transfers payment of $\frac{\ln \frac{\lambda_{s} \gamma_{s}}{\lambda_{r} \gamma_{r}}}{\gamma_{r}+\gamma_{s}}$ to the riskaverse supplier. With $\lambda_{s}$ increasing or $\lambda_{r}$ decreasing, the transfer payment to the risk-averse supplier increases. Once $\frac{\lambda_{s}}{\lambda_{r}}$ increases up to $\frac{\gamma_{r}}{\gamma_{s}} \exp ^{\gamma_{s} \Delta \pi}$, the transfer payment to the risk-averse supplier is $\frac{\gamma_{s}}{\gamma_{r}+\gamma_{s}} \Delta \pi$. If the riskaverse supplier's negotiation power relative to the risk-averse retailer's is strong (i.e., $\frac{\lambda_{s}}{\lambda_{r}} \geq \frac{\gamma_{r}}{\gamma_{s}} \exp ^{\gamma_{s} \Delta \pi}$ ), the risk-averse supplier would occupy the entire additional supply chain profit.

Example 6.3. We consider a supply chain consisting of a risk-neutral supplier with the utility function

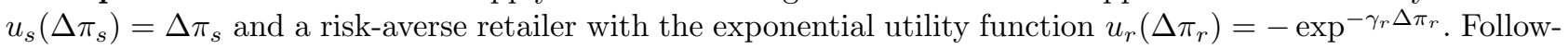
ing the linear aggregation rule, the supply-chain utility function is

$$
u\left(\Delta \pi_{r}, \Delta \pi_{s}\right)=-\lambda_{r} \exp ^{-\gamma_{r} \Delta \pi_{r}}+\lambda_{s} \Delta \pi_{s}:=u^{2} .
$$

Solving the program (P11) with $u^{2}$, following lemma establishes the optimal risk-sharing proportion of the centralized supply chain with a risk-averse retailer.

Lemma 6.4. When only the retailer is risk-averse,

(i) If $\frac{\lambda_{r}}{\lambda_{s}} \leq \frac{1}{\gamma_{r}}$, then $\rho^{*}=\rho_{\min }, \Delta \pi_{r}=0$ and $\Delta \pi_{s}=\Delta \pi$;

(ii) If $\frac{1}{\gamma_{r}}<\frac{\lambda_{r}}{\lambda_{s}}<\frac{\exp \gamma_{r} \Delta \pi}{\gamma_{r}}$, then $\rho^{*}=\rho_{\min }+\frac{\ln \frac{\lambda_{r} \gamma_{r}}{\lambda_{s}}}{\gamma_{r}}, \Delta \pi_{r}=\frac{\ln \frac{\lambda_{r} \gamma_{r}}{\lambda_{s}}}{\gamma_{r}}$ and $\Delta \pi_{s}=\Delta \pi-\frac{\ln \frac{\lambda_{r} \gamma_{r}}{\lambda_{s}}}{\gamma_{r}}$;

(iii) If $\frac{\lambda_{r}}{\lambda_{s}} \geq \frac{\exp ^{\gamma_{r} \Delta \pi}}{\gamma_{r}}$, then $\rho^{*}=\rho_{\max }, \Delta \pi_{r}=\Delta \pi$ and $\Delta \pi_{s}=0$.

Lemma 6.4 shows that, any given $\gamma_{r}$, if the risk-averse retailer's negotiation power relative to the risk-neutral supplier's is weak (i.e., $\frac{\lambda_{r}}{\lambda_{s}} \leq \frac{1}{\gamma_{r}}$ ), the risk-neutral supplier would occupy the entire additional supply-chain profit. If the risk-averse retailer's negotiation power relative to the risk-neutral supplier's is moderate (i.e., $\frac{1}{\gamma_{r}}<\frac{\lambda_{r}}{\lambda_{s}}<\frac{\exp ^{\gamma_{r} \Delta \pi}}{\gamma_{r}}$ ), the risk-neutral supplier would occupy the entire additional supply-chain profit and then transfer payment of $\frac{\ln \frac{\lambda_{r} \gamma_{r}}{\lambda_{s}}}{\gamma_{r}}$ to the risk-averse retailer. With $\lambda_{r}$ increasing or $\lambda_{s}$ decreasing, the risk-averse retailer's negotiation power relative to the risk-neutral supplier increases. Once $\frac{\lambda_{s}}{\lambda_{s}}$ increases up to $\frac{\exp ^{\gamma_{r} \Delta \pi}}{\gamma_{r}}$, the transfer payment to the risk-averse retailer is $\Delta \pi$. If the risk-averse retailer's negotiation power relative to the risk-neutral supplier is strong (i.e., $\frac{\lambda_{r}}{\lambda_{s}} \geq \frac{\exp ^{\gamma_{r} \Delta \pi}}{\gamma_{r}}$ ), the risk-averse retailer would occupy the entire additional supply-chain profit. 
TABLE 4. Optimal quantity decisions with uniform-uniform distribution.

\begin{tabular}{|c|c|c|c|}
\hline Model & Ordering quantity in Stage 2 & & Production quantity in Stage 1 \\
\hline Centralized & $\begin{aligned} Q_{r}^{\mathrm{SC}^{*}} & =i-\frac{\beta}{2}+\frac{p-v_{s}}{p-v_{r}} \beta \\
Q_{r}^{\mathrm{SC}^{*}} & =Q_{s}^{\mathrm{SC}^{*}}\end{aligned}$ & $\begin{array}{l}\text { if } i \leq i_{Q_{s}^{S C^{*}}} \\
\text { if } i_{Q_{s}^{S S^{*}}} \leq i\end{array}$ & $Q_{r}^{S C^{*}}=\gamma+\frac{\alpha-\beta}{2}+\frac{p-v_{s}}{p-v_{r}} \beta-\sqrt{\frac{2\left(c-v_{s}\right) \alpha \beta}{p-v_{r}}}$ \\
\hline NRS & $\begin{aligned} Q_{r}^{N^{*}} & =i-\frac{\beta}{2}+\frac{p-w}{p-v_{r}} \beta \\
Q_{r}^{N^{*}} & =Q_{s}^{N^{*}}\end{aligned}$ & $\begin{array}{l}\text { if } i \leq i_{Q_{s}^{N^{*}}} \\
\text { if } i_{Q_{s}^{N^{*}}} \leq i\end{array}$ & $Q_{r}^{N^{*}}=\gamma+\frac{\alpha-\beta}{2}+\frac{p-w}{p-v_{r}} \beta-\frac{c-v_{s}}{w-v_{s}} \alpha$ \\
\hline ORS-1 & $\begin{array}{l}Q_{r}^{S 1^{*}}=i-\frac{\beta}{2}+\frac{p-w+\rho\left(c-v_{s}\right)}{p-v_{r}} \beta \\
Q_{r}^{S 1^{*}}=Q_{s}^{S 1^{*}}\end{array}$ & $\begin{array}{l}\text { if } i \leq i_{Q_{s}^{S 1^{*}}} \\
\text { if } i_{Q_{s}^{S 1^{*}}} \leq i\end{array}$ & $\begin{aligned} Q_{r}^{S 1^{*}}= & \gamma+\frac{\alpha-\beta}{2}+\frac{p-w+\rho\left(c-v_{s}\right)}{p-v_{r}} \beta \\
& -\frac{(1-\rho)\left(c-v_{s}\right)}{w-v_{s}-\rho\left(c-v_{s}\right)} \alpha\end{aligned}$ \\
\hline ORS-2 & $\begin{array}{l}Q_{r}^{S 2^{*}}=i-\frac{\beta}{2}+\frac{p-w}{p-v_{r}-\phi\left(w-v_{r}\right)} \beta \\
Q_{r}^{S 2^{*}}=Q_{s}^{S 2^{*}}\end{array}$ & $\begin{array}{l}\text { if } i \leq i_{Q_{s}^{S 2^{*}}} \\
\text { if } i_{Q_{s}^{S 2^{*}}} \leq i\end{array}$ & $Q_{r}^{S 2^{*}}=\gamma+\frac{\alpha-\beta}{2}+\frac{w-v_{s}}{\phi\left(w-v_{r}\right)} \beta-A 1$ \\
\hline BRS & $\begin{array}{l}Q_{r}^{B^{*}}=i-\frac{\beta}{2}+\frac{p-w+\rho\left(c-v_{s}\right)}{p-v_{r}-\phi\left(w-v_{r}\right)} \beta \\
Q_{r}^{B^{*}}=Q_{s}^{B^{*}}\end{array}$ & $\begin{array}{l}\text { if } i \leq i_{Q_{s}^{B^{*}}} \\
\text { if } i_{Q_{s}^{B^{*}}} \leq i\end{array}$ & $Q_{r}^{B^{*}}=\gamma+\frac{\alpha-\beta}{2}+\frac{w-v_{s}-\rho\left(c-v_{s}\right)}{\phi\left(w-v_{r}\right)} \beta-A 2$ \\
\hline
\end{tabular}

Example 6.5. We consider a supply chain consisting of a risk-averse supplier with exponential utility function $u_{s}\left(\Delta \pi_{s}\right)=-\exp ^{-\gamma_{s} \Delta \pi_{s}}$ and a risk-neutral retailer with the utility function $u_{r}\left(\Delta \pi_{r}\right)=\Delta \pi_{r}$. Following the linear aggregation rule, the supply chain utility function is

$$
u\left(\Delta \pi_{r}, \Delta \pi_{s}\right)=\lambda_{r} \Delta \pi_{r}-\lambda_{s} \exp ^{-\gamma_{s} \Delta \pi_{s}}=u^{3} .
$$

Solving the program (P11) with $u^{3}$, the following lemma establishes the optimal risk-sharing proportion of the centralized supply chain with a risk-averse supplier.

Lemma 6.6. When only the supplier is risk-averse,

(i) If $\frac{\lambda_{s}}{\lambda_{r}} \leq \frac{1}{\gamma_{s}}$, then $\rho^{*}=\rho_{\max }, \Delta \pi_{r}=\Delta \pi$ and $\Delta \pi_{s}=0$;

(ii) If $\frac{1}{\gamma_{s}} \leq \frac{\lambda_{s}}{\lambda_{r}} \leq \frac{1}{\gamma_{s}} \exp ^{\gamma_{s} \Delta \pi}$, then $\rho^{*}=\rho_{\max }-\frac{\ln \frac{\lambda_{s} \gamma_{s}}{\lambda_{r}}}{\gamma_{s}}, \Delta \pi_{r}=\Delta \pi-\frac{\ln \frac{\lambda_{s} \gamma_{s}}{\lambda_{r}}}{\gamma_{s}}$ and $\Delta \pi_{s}=\frac{\ln \frac{\lambda_{s} \gamma_{s}}{\lambda_{r}}}{\gamma_{s}}$;

(iii) If $\frac{\lambda_{s}}{\lambda_{r}} \geq \frac{1}{\gamma_{s}} \exp ^{\gamma_{s} \Delta \pi}$, then $\rho^{*}=\rho_{\min }, \Delta \pi_{r}=0$ and $\Delta \pi_{s}=\Delta \pi$.

Lemma 6.6 shows that, compared with the risk-neutral retailer, the risk-averse supplier with stronger risk aversion obtains greater additional supply chain profit. The supplier needs a long lead time to prepare production. Through updating demand information, the retailer may improve demand forecast to make more precise final order decision. So, the supplier likely suffers from heavier overproduction loss. To make up the potential loss, the risk-averse supplier requires greater additional profit.

\section{NUMERICAL ANALYSiS}

Numerical examples are presented to illustrate the main theoretical results. For the sake of simplicity, the optimal quantity decisions with uniform-uniform distribution in Table 4 with $A 1=$ $\sqrt{\left[\frac{w-v_{s}}{\phi\left(w-v_{r}\right)} \beta-\frac{p-w}{p-v_{r}-\phi\left(w-v_{r}\right)} \beta\right]^{2}+\frac{2\left(c-v_{s}\right) \alpha \beta}{\phi\left(w-v_{r}\right)}}$ and $A 2=\sqrt{\left[\frac{w-v_{s}-\rho\left(c-v_{s}\right)}{\phi\left(w-v_{r}\right)} \beta-\frac{p-w}{p-v_{r}-\phi\left(w-v_{r}\right)} \beta\right]^{2}+\frac{2(1-\rho)\left(c-v_{s}\right) \alpha \beta}{\phi\left(w-v_{r}\right)}}$. Similar to Huang et al. [21], Chen et al. [9] and Wang and Tsao [41], the main parameters are set as follows: $p=200, c=50, v_{s}=30, v_{r}=20, \alpha=1000, \beta=800$, and $\gamma=1500$. 
TABLE 5. Optimal quantity decisions and expected profits in the BRS model.

\begin{tabular}{llllllllll}
\hline \hline$w$ & $\rho$ & $\phi$ & $Q_{s}^{B}$ & $Q_{r}^{B} \mid i$ & $\pi^{B}$ & $\pi_{r}^{B}$ & $\pi_{s}^{B}$ & $\pi_{r}^{B} / \pi^{\mathrm{SC}}$ & $\pi_{s}^{B} / \pi^{\mathrm{SC}}$ \\
\hline 184 & 0.1 & 0.98 & 1933 & $i+355$ & 2.0973 & 0.2097 & 1.8876 & 0.1 & 0.9 \\
168 & 0.2 & 0.97 & 1933 & $i+355$ & 2.0973 & 0.4194 & 1.6779 & 0.2 & 0.8 \\
152 & 0.3 & 0.95 & 1933 & $i+355$ & 2.0973 & 0.6291 & 1.4682 & 0.3 & 0.7 \\
136 & 0.4 & 0.93 & 1933 & $i+355$ & 2.0973 & 0.8389 & 1.2584 & 0.4 & 0.6 \\
120 & 0.5 & 0.90 & 1933 & $i+355$ & 2.0973 & 1.0486 & 1.0487 & 0.5 & 0.5 \\
104 & 0.6 & 0.85 & 1933 & $i+355$ & 2.0973 & 1.2583 & 0.8380 & 0.6 & 0.4 \\
88 & 0.7 & 0.79 & 1933 & $i+355$ & 2.0973 & 1.4681 & 0.6292 & 0.7 & 0.3 \\
72 & 0.8 & 0.69 & 1933 & $i+355$ & 2.0973 & 1.6778 & 0.4195 & 0.8 & 0.2 \\
56 & 0.9 & 0.50 & 1933 & $i+355$ & 2.0973 & 1.8875 & 0.2098 & 0.9 & 0.1 \\
\hline
\end{tabular}

\subsection{The supply-chain coordination}

In this subsection, we first verify that how the bi-directional risk-sharing contract coordinates the supply chain with demand information updating. To be more specific, in the centralized model, the supply chain's optimal quantity decisions are: at the beginning of Stage 1, the supplier's optimal production quantity is $Q_{s}^{\mathrm{SC}^{*}}=1933$. The critical demand information threshold is $i_{Q_{s}^{S C^{*}}}=1578$. At the end of Stage 1 , the latest demand information $I$ is realized as $i$ upon demand information updating. If $1000 \leq i \leq 1578$, the optimal ordering quantity is $Q_{r}^{\mathrm{SC}^{*}}=i+355$; and if $1578<i \leq 2000, Q_{r}^{\mathrm{SC}^{*}}=1933$. The supply chain expected profit is $\pi^{\mathrm{SC}}=2.0973 \times 10^{5}$ (see Props. 4.1, 4.3 and Cor. 4.2 in Sect. 4.1).

In the BRS model with $\{w, \rho, \phi\}$, given the values of $\rho$ range from 0.1 to 0.9 , then the values of $w$ and $\phi$ can be obtained from Proposition 5.2. Then, the feasible intervals of $w, \rho$ and $\phi$ are $(50,200),(0,1)$ and $(0,1)$, respectively. Table 5 presents the optimal quantity decisions and the expected profits with the bi-directional risk-sharing contract $\{w, \rho, \phi\}$. For example, if $\rho=0.3$, then $w=152$ and $\phi=0.95$. The supplier charges a wholesale price of 152 , and bears $70 \%$ of overproduction risk and shares $5 \%$ of overstock risk with the retailer. Correspondingly, the retailer shares $30 \%$ of overproduction risk with the supplier and bears $95 \%$ of overstock risk. Under such settings, $Q_{s}^{\mathrm{SC}^{*}}=Q_{s}^{B^{*}}$ and $Q_{r}^{\mathrm{SC}}=Q_{r}^{B^{*}}$. So that the supply chain members' quantity decisions reach system-optimal level. The optimal expected profits of the supplier and retailer are $\pi_{s}^{B}=1.4681 \times 10^{5}$ and $\pi_{r}^{B}=0.6292 \times 10^{5}$, respectively. Note that $\pi^{B}=\pi_{r}^{B}+\pi_{s}^{B}=\pi^{\mathrm{SC}}=2.0973 \times 10^{5}$, which means that the coordination of the supply chain is achieved. In addition, $\pi_{r}^{B}=0.3 \pi^{\mathrm{SC}}$ and $\pi_{s}^{B}=0.7 \pi^{\mathrm{SC}}$. It indicates that $\rho$ and $1-\rho$ are the proportions of the retailer's and the supplier's profit to allocate the supply-chain profit, respectively. According to the Table 5, by tuning $\rho$, the supply-chain profit can be arbitrary allocated between both members. Therefore, a bi-directional contract $\{w, \rho, \phi\} \in M$ can achieve the supply chain coordination with demand information updating (Props. 4.13, 4.15, Cor. 4.14 in Sect. 4.2 .4 and Prop. 5.2 in Sect. 5).

\subsection{Performance comparison among four models}

In this subsection, by comparing the supply chain's optimal expected profit in the coordinating contract (i.e., BRS contract) with non-coordinating contracts (i.e., NRS, ORS-1 and ORS-2 contracts), we further evaluate the performance of the proposed contract. Specific as follows:

(1) In the NRS model with $\{w\}$, the supplier's production quantity and the retailer's ordering quantity depend on wholesale price $w$, which is given and kept the same as $\{w, \rho, \phi\}$. Table 6 shows the supply chain optimal quantity decisions and expected profit under $\{w\}$. For example, given $w=152$ in Table 6 , the supplier's optimal production quantity of Stage 1 is $Q_{s}^{N}=1640$. The critical information threshold is $i_{Q_{s}^{N}}=1840$. Upon observing $i$, the retailer's optimal ordering quantity $Q_{r}^{N}$ of Stage 2 is: if $1000 \leq i \leq 1840$, then $Q_{r}^{N}=i-200$, and if $1840 \leq i \leq 2000$, then $Q_{r}^{N}=1640$. The optimal expected profits of the supply chain and members are $\pi^{N}=0.6200 \times 10^{5}, \pi_{s}^{N}=0.0820 \times 10^{5}$ and $\pi_{r}^{N}=0.5380 \times 10^{5}$, respectively. In 
TABLE 6. Optimal quantity decisions and expected profits in the ORS model.

\begin{tabular}{lllllllll}
\hline \hline$w$ & $Q_{s}^{N}$ & $Q_{r}^{N} \mid i$ & $\pi^{N}$ & $\Delta \pi$ & $\pi_{s}^{N}$ & $\operatorname{Gap}_{s}^{N}$ & $\pi_{r}^{N}$ & $\mathrm{Gap}_{r}^{N}$ \\
\hline 184 & 1537 & $i-334$ & 0.3340 & 1.7633 & 0.165 & 0.210 & 0.169 & 0.191 \\
168 & 1590 & $i-267$ & 0.4720 & 1.6253 & 0.124 & 0.704 & 0.348 & 0.169 \\
152 & 1640 & $i-200$ & 0.6200 & 1.4773 & 0.082 & 0.869 & 0.538 & 0.145 \\
136 & 1684 & $i-134$ & 0.7700 & 1.3273 & 0.033 & 0.961 & 0.737 & 0.121 \\
120 & 1722 & $i-67$ & 0.9290 & 1.1683 & -0.017 & 1.016 & 0.946 & 0.097 \\
104 & 1750 & $i$ & 1.0930 & 1.0043 & -0.071 & 1.056 & 1.164 & 0.074 \\
88 & 1759 & $\mathrm{i}+67$ & 1.2630 & 0.8343 & -0.126 & 1.085 & 1.389 & 0.053 \\
72 & 1733 & $\mathrm{i}+133$ & 1.4330 & 0.6643 & -0.183 & 1.109 & 1.616 & 0.036 \\
56 & 1628 & $\mathrm{i}+200$ & 1.5850 & 0.5123 & -0.235 & 1.124 & 1.820 & 0.035 \\
\hline
\end{tabular}

TABLE 7. Optimal quantity decisions and expected profits in the ORS-1 model.

\begin{tabular}{llllllllll}
\hline \hline$w$ & $\rho$ & $Q_{s}^{S 1}$ & $Q_{r}^{S 1} \mid i$ & $\pi^{S 1}$ & $\Delta \pi$ & $\pi_{s}^{S 1}$ & $\mathrm{Gap}_{s}^{S 1}$ & $\pi_{r}^{S 1}$ & $\mathrm{Gap}_{r}^{S 1}$ \\
\hline 184 & 0.1 & 1558 & $i-324$ & 1.6693 & 0.4280 & 1.5076 & 0.201 & 0.1617 & 0.226 \\
168 & 0.2 & 1633 & $i-248$ & 1.7645 & 0.3328 & 1.4307 & 0.147 & 0.3338 & 0.203 \\
152 & 0.3 & 1709 & $i-173$ & 1.8468 & 0.2505 & 1.3312 & 0.093 & 0.5156 & 0.180 \\
136 & 0.4 & 1784 & $i-97.8$ & 1.9164 & 0.1809 & 1.2091 & 0.039 & 0.7073 & 0.156 \\
120 & 0.5 & 1860 & $i-22.2$ & 1.9731 & 0.1242 & 1.0642 & $-\mathbf{0 . 0 1 5}$ & 0.9089 & 0.132 \\
104 & 0.6 & 1935 & $i+53.3$ & 2.0170 & 0.0803 & 0.8967 & $\mathbf{- 0 . 0 7 0}$ & 1.1203 & 0.109 \\
88 & 0.7 & 2011 & $i+128$ & 2.0480 & 0.493 & 0.7065 & $\mathbf{- 0 . 1 2 3}$ & 1.3415 & 0.086 \\
72 & 0.8 & 2086 & $i+204$ & 2.0662 & 0.0311 & 0.4936 & $\mathbf{- 0 . 1 7 8}$ & 1.5726 & 0.062 \\
56 & 0.9 & 2162 & $i+280$ & 2.0715 & 0.0258 & 0.2582 & $\mathbf{- 0 . 2 3 5}$ & 1.8133 & 0.039 \\
\hline
\end{tabular}

particular, $\operatorname{Gap}_{s}^{N}>0$ and $\operatorname{Gap}_{r}^{N}>0$, which suggests that the BRS contract is always superior than the NRS contract for any $\rho \in[0,1]$. The NRS contract cannot coordinate the supply chain unless $w=c$. The retailer, however, would occupy all profit (see Props. 4.4, 4.6 and Cor. 4.5 in Sect. 4.2.1).

(2) In the ORS-1 model with $\{w, \rho\}$, the supply chain's optimal quantity decisions and expected profits depend on the wholesale price $w$ and sharing proportion of overproduction $\rho$, which is given and kept the same as $\{w, \rho, \phi\}$ in Table 7. Take $w=152$ with $\rho=0.3$ as an example, at the beginning of Stage 1 , the supplier's optimal production quantity is $Q_{s}^{S 1}=1709$. The critical information threshold is $i_{Q_{s}^{S 1}}=1882$. When the latest demand information $I=i$ is observed, the retailer's optimal ordering quantity at the beginning of Stage 2 is given as follows: if $1000 \leq i \leq 1882$, then $Q_{r}^{S 1}=i-173$, and if $1882 \leq i \leq 2000$, then $Q_{r}^{S 1}=1709$. The optimal expected profits of the supply chain and members are $\pi^{S 1}=1.8468 \times 10^{5}, \pi_{s}^{S 1}=1.3312 \times 10^{5}$ and $\pi_{r}^{S 1}=0.5156 \times 10^{5}$, respectively. Since $\Delta \pi=\pi^{\mathrm{SC}}-\pi^{S 1}=0.2505 \times 10^{5}>0$, the expected profit in the ORS-1 model cannot reach the system-optimal level. In addition, $\operatorname{Gap}_{r}^{S 1}>0$ suggests that the retailer always benefits from the BRS contract than the ORS- 1 contract for any $\rho \in[0,1]$. However, Gap ${ }_{s}^{S 1}>0$ only when $\rho \in\left[0, \rho_{\mathrm{min}}^{S 1}\right]$, the supplier benefits from the BRS contract than the ORS-1 contract. (see Props. 4.7, 4.9 and Cor. 4.8 in Sect. 4.2.2).

(3) In the ORS-2 model with $\{w, \pi\}$, the supply chain's optimal quantity decisions and expected profits are influenced by both the wholesale price $w$ and sharing proportion of overstock $\phi$, which is given and kept the same as $\{w, \rho, \phi\}$ in Table 8. Similarly, take $w=152$ with $\phi=0.95$ as an example, at the beginning of Stage 1 , the supplier's optimal production quantity is $Q_{s}^{S 2}=1873$. The critical information threshold is $i_{Q_{s}^{S 2}}=$ 1607. When the latest demand information $I=i$ is observed, the retailer's optimal ordering quantity at the beginning of Stage 2 is given as follows: if $1000 \leq i \leq 1607$, then $Q_{r}^{S 2}=i+266$, and if $1607 \leq i \leq 2000$, then 
TABLE 8. Optimal quantity decisions and expected profits in the ORS-2 model.

\begin{tabular}{llllllllll}
\hline \hline$w$ & $\phi$ & $Q_{s}^{S 2}$ & $Q_{r}^{S 2} \mid i$ & $\pi^{S 2}$ & $\Delta \pi$ & $\pi_{s}^{S 2}$ & $\mathrm{Gap}_{s}^{S 2}$ & $\pi_{r}^{S 2}$ & $\mathrm{Gap}_{r}^{S 2}$ \\
\hline 184 & 0.98 & 1910 & $i+266$ & 2.0915 & 0.0058 & 1.8782 & 0.004 & 0.2133 & $\mathbf{- 0 . 0 0 2}$ \\
168 & 0.97 & 1893 & $i+266$ & 2.0912 & 0.0061 & 1.6651 & 0.007 & 0.4261 & $\mathbf{- 0 . 0 0 4}$ \\
152 & 0.95 & 1873 & $i+266$ & 2.0904 & 0.0069 & 1.4522 & 0.011 & 0.6382 & $\mathbf{- 0 . 0 0 5}$ \\
136 & 0.93 & 1850 & $i+266$ & 2.0889 & 0.0084 & 1.2396 & 0.014 & 0.8493 & $\mathbf{- 0 . 0 0 6}$ \\
120 & 0.90 & 1822 & $i+266$ & 2.0861 & 0.0112 & 1.0276 & 0.019 & 1.0585 & $\mathbf{- 0 . 0 0 5}$ \\
104 & 0.85 & 1786 & $i+266$ & 2.0814 & 0.0159 & 0.8164 & 0.025 & 1.2650 & $\mathbf{- 0 . 0 0 4}$ \\
88 & 0.79 & 1738 & $i+266$ & 2.0723 & 0.0250 & 0.6062 & 0.036 & 1.4661 & 0.001 \\
72 & 0.69 & 1668 & $i+266$ & 2.0537 & 0.0436 & 0.3978 & 0.051 & 1.6559 & 0.011 \\
56 & 0.50 & 1553 & $i+266$ & 2.0056 & 0.0917 & 0.1932 & 0.075 & 1.8124 & 0.039 \\
\hline
\end{tabular}

$Q_{r}^{S 2}=1873$. The optimal expected profits of the supply chain and both members are $\pi^{S 2}=2.0904 \times 10^{5}$, $\pi_{s}^{S 2}=1.4522 \times 10^{5}$ and $\pi_{r}^{S 2}=0.6382 \times 10^{5}$, respectively. Since $\Delta \pi=\pi^{\mathrm{SC}}-\pi^{S 2}=0.011 \times 10^{5}>0$, the expected profit in the ORS-2 model also cannot reach the system-optimal level. In addition, Gap $\operatorname{Gi}_{s}^{S 2}>0$ shows that the supplier always benefits from the BRS contract than the ORS-2 contract for any $\rho \in[0,1]$. However, $\operatorname{Gap}_{r}^{S 2}>0$ only when $\rho \in\left[\rho_{\max }^{S 2}, 1\right]$, the retailer benefits from the BRS contract than the ORS-2 contract (see Props. 4.10, 4.12 and Cor. 4.11 in Sect. 4.2.3).

\subsection{The impacts of the contract parameters}

In this subsection, taking the NRS contract as a benchmark, we study the impacts of $\rho, \phi, p, c, v_{s}$, and $v_{r}$ on the expected profits of the supply chain and members in the BRS contract. The results are illustrated in Figures 3 and 4 . When analyzing the influence of a certain parameter, the values of other parameters maintain unchanged.

Figure 3 shows the impacts of $\rho$ and $\phi$ on the expected profits of the supply chain and members. The values of $\rho$ and $\phi$ both range from 0.1 to 0.9. The wholesale price is $w=100$. In Figure 2(1), $\phi=0.5$ denotes the supplier shares $50 \%$ of overstock risk. In Figure 2(2), $\rho=0.5$ denotes the retailer shares $50 \%$ overproduction risk. From Figure 3, the retailer's expected profit decreases as $\rho$ but increases as $\phi$, in sharp contrast to the supplier's tendency. As $\rho$ or $\phi$ increases, the supply-chain expected profit first increases and then decreases. To be more specific, when $w=100$ and $\phi=0.5, \pi^{B}$ increases in $\rho \in(0.1,0.6)$ and decreases in $\rho \in(0.6,0.9)$. Since there exists double marginal effects, only adjusting $\rho$ or $\phi$ cannot achieve optimal supply chain's expected profit unless $\{w, \rho, \phi\} \in M$.

Figure 4 presents the impacts of the parameters $p, c, v_{s}$ and $v_{r}$ on the expected profits of the supply chain. When $w=120, \rho=0.5$ and $\phi=0.90$, we separately adjust $p, c, v_{s}$ and $v_{m}$ from $110,35,21,0$ to 200, 80, 48, 27. From Figure 4, the supply-chain expected profits both increase with $p$ and $v_{s}$ and both decrease with $c$ and $v_{r}$. The high $p, c$ and $v_{r}$ and the low $v_{s}$ enlarge the gap between the supply chain profit curves of $\{w, \rho, \phi\} \in M$ and $\{w\}$. That suggests that $\{w, \rho, \phi\} \in M$ is more beneficial to the supply chain for the high retail price, production cost and final salvage value, and the low initial salvage value.

\subsection{The impacts of risk aversion and negotiation power}

In this subsection, we further study the impacts of risk aversion and negotiation power on the optimal quantity decisions and the allocation of the additional supply-chain profit with $\{w, \rho, \phi\} \in M$. Take the NRS contract $\{w\}$ as the benchmark, when $w=150$, the additional supply chain profit $\Delta \pi=1.4279 \times 10^{5}$. Specially, only when $\rho \in[0.2879,1]$, both the supplier and the retailer accept the proposed contract.

Example 7.1. Figure 5 presents the impacts of the members' negotiation power on the optimal contract and the allocation of the additional supply-chain profit when both members are risk-averse. When $\gamma_{r}=\gamma_{s}=1$ : 


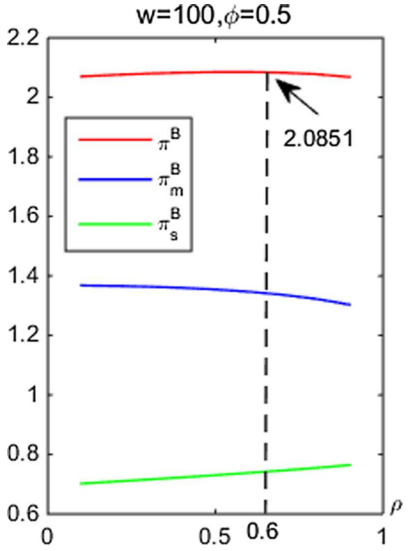

(1)

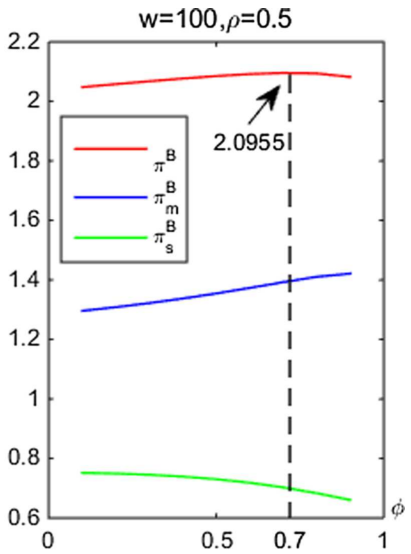

(2)

Figure 3. The impacts of $\rho$ and $\phi$ on $\pi^{B}, \pi_{s}^{B}$ and $\pi_{r}^{B}$.
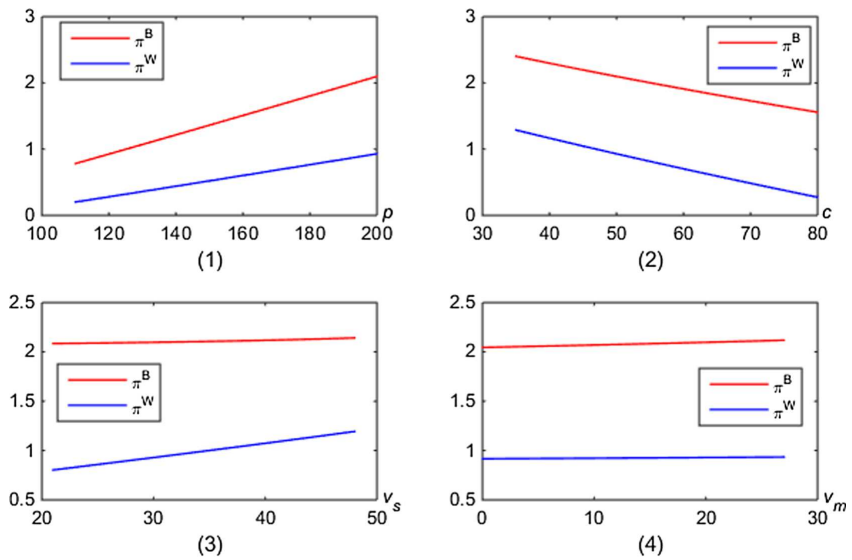

Figure 4. The impacts of $p, c, v_{s}$ and $v_{r}$ on $\pi^{B}$ and $\pi^{N}$.

(1) if $0 \leq \lambda_{s} \leq 0.1934$, then $\rho^{*}=1, \Delta \pi_{r}=1.4279$ and $\Delta \pi_{s}=0 ;(2)$ if $0.1934 \leq \lambda_{s} \leq 0.8066$, then $\rho^{*}=$ $0.644-\frac{\ln \lambda_{s}-\ln \left(1-\lambda_{s}\right)}{4.1946}, \Delta \pi_{r}=0.7139-\frac{\ln \lambda_{s}-\ln \left(1-\lambda_{s}\right)}{2}$ and $\Delta \pi_{s}=0.7139+\frac{\ln \lambda_{s}-\ln \left(1-\lambda_{s}\right)}{2}$; and (3) if $0.8066 \leq \lambda_{s} \leq 1$, then $\rho^{*}=0.2879, \Delta \pi_{r}=0$ and $\Delta \pi_{s}=1.4279$. That suggests that if both members have the same absolute risk aversion, they may gain the equal proportion of $\Delta \pi$. There exists transfer payment between both members, depending on the members' negotiation power. The stronger negotiation power relative to the retailer brings the supplier greater transfer payment.

Figure 6 shows the impact of the members' risk aversion on the optimal decisions and the additional supplychain profit allocation when both members have the same negotiation power. Without loss of generality, we study three scenarios of risk aversion: (1) $\gamma_{r}<\gamma_{s}$; (2) $\gamma_{r}=\gamma_{s}$; and (3) $\gamma_{r}>\gamma_{s}$. To be more specific, when $\lambda_{r}=\lambda_{s}$, if $2 \gamma_{r}=\gamma_{s}$ and $\gamma_{r} \geq 0.24272$, then $\rho^{*}=0.7626-\frac{\ln 2}{6.2919 \gamma_{r}}, \Delta \pi_{r}=0.95193-\frac{\ln 2}{3 \gamma_{r}}$ and $\Delta \pi_{s}=0.47597+\frac{\ln 2}{3 \gamma_{r}}$ (see Fig. 6). As $\gamma_{r}$ increase, $\rho^{*}$ and $\Delta \pi_{r}$ increases while $\Delta \pi_{s}$ decreases. It suggests that if the supplier has stronger risk aversion relative to the retailer $\left(e . g ., 2 \gamma_{r}=\gamma_{s}\right)$, the retailer gains the additional supply-chain profit of 0.95193 while the supplier gains 0.47597 . At the same time, the retailer transfers payment of $\frac{\ln 2}{3 \gamma_{r}}$ to the supplier, which decreases as $\gamma_{r}$ increases. The retailer with stronger risk aversion transfers less payment to 


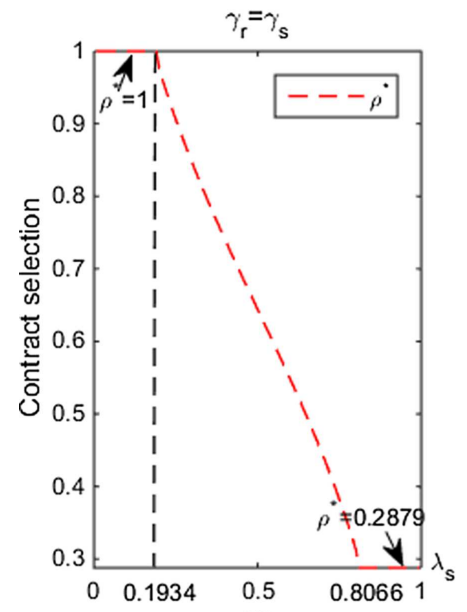

(1)

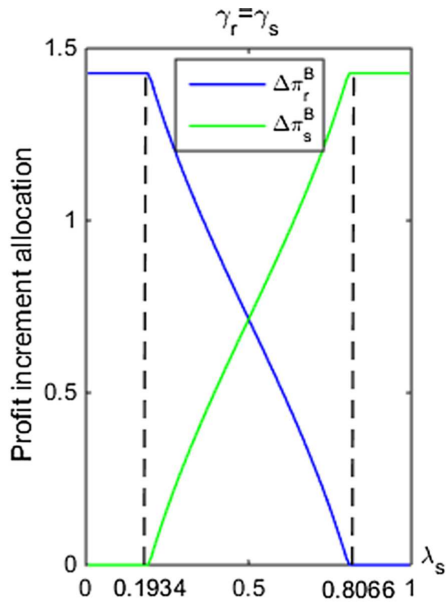

(2)

Figure 5. The optimal allocation of $\Delta \pi$ with $\lambda_{s}$.
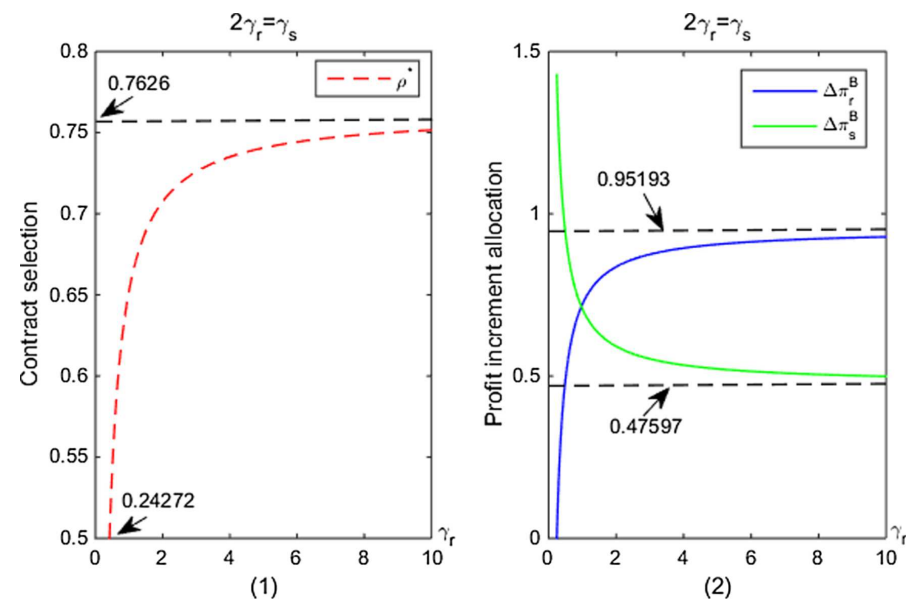

FiguRE 6 . The optimal allocation of $\Delta \pi$ with $\lambda_{r}<\lambda_{s}$.

the supplier. If $\gamma_{r}=\gamma_{s}>0$, then $\rho^{*}=\frac{\lambda_{\min }+\lambda_{\max }}{2}=0.644$ and $\Delta \pi_{r}=\Delta \pi_{s}=\frac{1}{2} \Delta \pi=0.7139$. It suggests that both members equally split $\Delta \pi$ when having the same risk aversion and negotiation power.

Figure 7 shows when $\gamma_{r}=2 \gamma_{s}$ and $\gamma_{r} \geq 0.4854$, then $\rho^{*}=0.5253+\frac{\ln 2}{6.2919 \gamma_{r}}, \Delta \pi_{r}=0.47597+\frac{\ln 2}{3 \gamma_{r}}$ and $\Delta \pi_{s}=0.95193-\frac{\ln 2}{3 \gamma_{r}}$. As $\gamma_{r}$ increase, $\rho^{*}$ and $\Delta \pi_{r}$ decreases while $\Delta \pi_{s}$ increases. The results are contrary to Figure 6. If the retailer has stronger risk aversion relative to the supplier $\left(e . g ., \gamma_{r}=2 \gamma_{s}\right)$, she obtains the extra expected profit of $\frac{1}{3} \Delta \pi$. Meanwhile, the retailer gets transfer payment of $\frac{\ln 2}{3 \gamma_{r}}$ from the supplier, which decreases as $\gamma_{r}$. Hence, the retailer with stronger risk aversion may gain more allocation from $\Delta \pi$. The retailer may not benefit from risk aversion.

Example 7.2. Figure 8 gives the impact of the retailer's negotiation power on the optimal decisions and the additional supply-chain profit allocation when only the retailer is risk-averse. From Figure 8 , when $\gamma_{r}=1$ : (1) if $\lambda_{r} \leq 0.5$, then $\rho^{*}=0.2879, \Delta \pi_{r}=0$ and $\Delta \pi_{s}=1.4279$; (2) if $0.5<\lambda_{r}<0.8066$, then $\rho^{*}=0.2879+$ $\frac{\ln \left(\lambda_{r}\right)-\ln \left(1-\lambda_{r}\right)}{2.0973}, \Delta \pi_{r}=\ln \frac{\lambda_{r}}{1-\lambda_{r}}$ and $\Delta \pi_{s}=1.4279-\ln \frac{\lambda_{r}}{1-\lambda_{r}}$; and (3) if $0.8066 \leq{ }_{r} \leq 1$, then $\rho^{*}=1, \Delta \pi_{r}=1.4279$ 

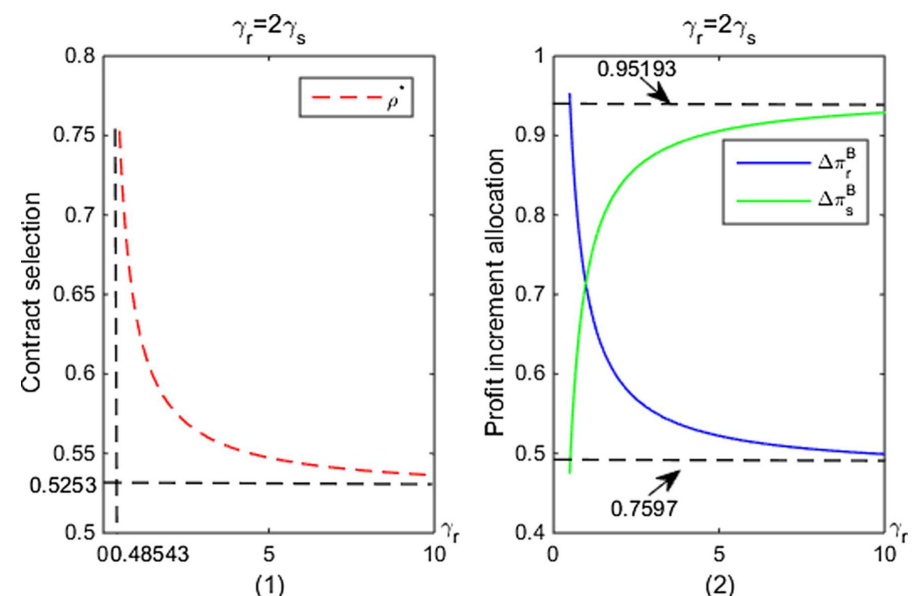

Figure 7 . The optimal allocation of $\Delta \pi$ with $\lambda_{r}>\lambda_{s}$.

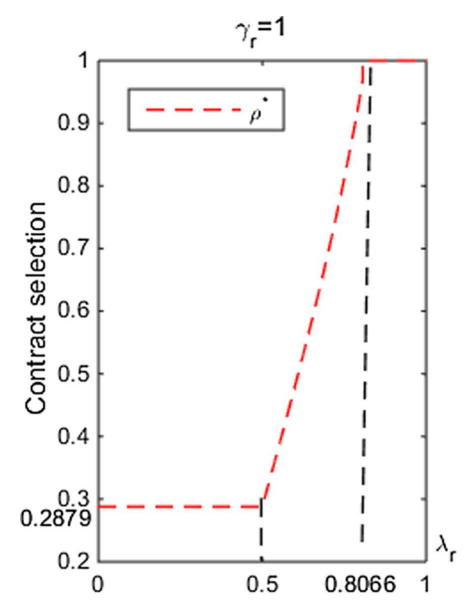

(1)

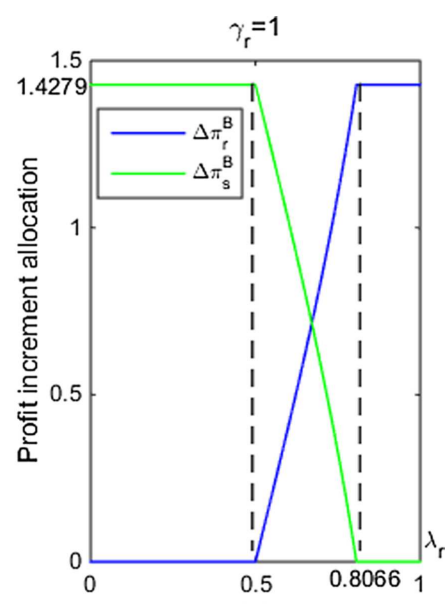

(2)

Figure 8 . The optimal allocation of $\Delta \pi$ with $\lambda_{r}$.

and $\Delta \pi_{s}=0$. The supplier occupies the entire $\Delta \pi$ when $\lambda_{r} \leq 0.5$. The retailer only obtains transfer payment of $\ln \frac{\lambda_{r}}{1-\lambda_{r}}$ from the supplier when $0.5<\lambda_{r}<0.8066$. This transfer payment $\ln \frac{\lambda_{r}}{1-\lambda_{r}}$ is increasing in $\lambda_{r}$. When $\lambda_{r}$ increases up to 0.8066 , the payment of the entire $\Delta \pi$ would be transferred to the retailer. That hints that when the retailer's negotiation power is strong enough relative to the supplier's (e.g., $\left.\lambda_{r} \geq 0.8066\right)$, the retailer would occupy the entire $\Delta \pi$.

Figure 9 shows the impact of the retailer's risk aversion on the optimal decisions and the additional supplychain expected profit allocation when both members have the same negotiation power. From Figure 9, if $\gamma_{r} \geq 1$ and $\lambda_{r}=\lambda_{s}$, then $\rho^{*}=0.2879+\frac{\ln \gamma_{r}}{2.0973 \gamma_{r}}, \Delta \pi_{r}=1.4279-\frac{\ln \gamma_{r}}{\gamma_{r}}$ and $\Delta \pi_{s}=\frac{\ln \gamma_{r}}{\gamma_{r}}$. Specifically, if $1 \gamma_{r} \leq 2.7183$, then $\rho^{*}$ and $\Delta \pi_{r}$ increase while $\Delta \pi_{s}$ decreases; and if $\gamma_{r} \geq 2.7183$, then $\rho^{*}$ and $\nu_{r} \pi_{r}$ decrease while $\Delta \pi_{s}$ increases. In the case that the retailer is risk-averse and the supplier is risk-neutral, the retailer only obtains transfer payment of $\frac{\ln \gamma_{r}}{\gamma_{r}}$ from the supplier, which is convex in $\gamma_{r}$. That is the risk aversion is beneficia for the retailer only when her risk-averse at a lower level (e.g., $1 \leq \gamma_{r} \leq 2.7183$ ). If the retailer raises her risk-averse at a higher level (e.g. $\left.\gamma_{r} \geq 2.7183\right)$, she will earn less or even no transfer payment from the supplier. The risk-neutral supplier 


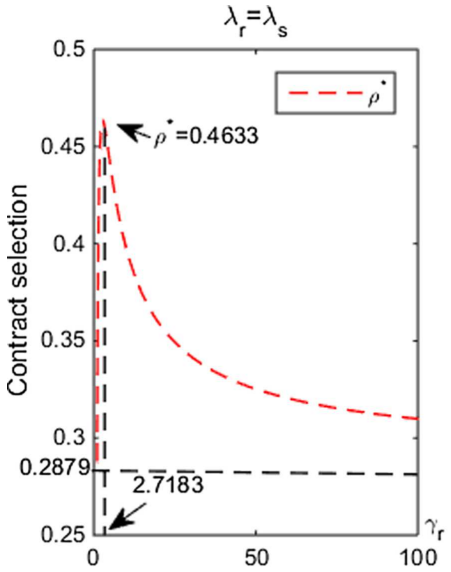

(1)

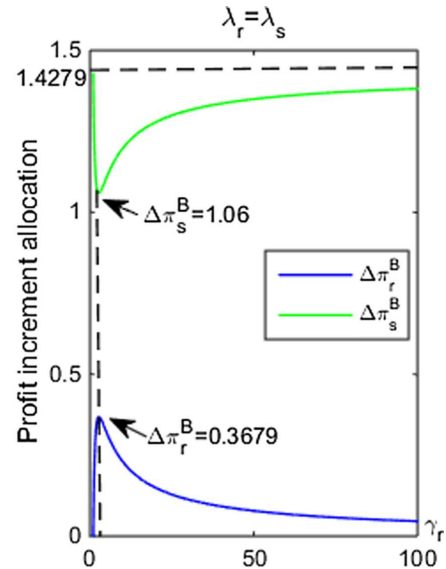

(2)

Figure 9. The optimal allocation of $\Delta \pi$ with $\gamma_{r}$.

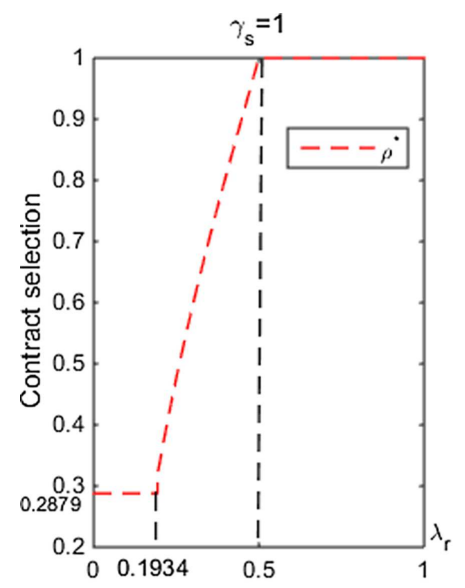

(1)

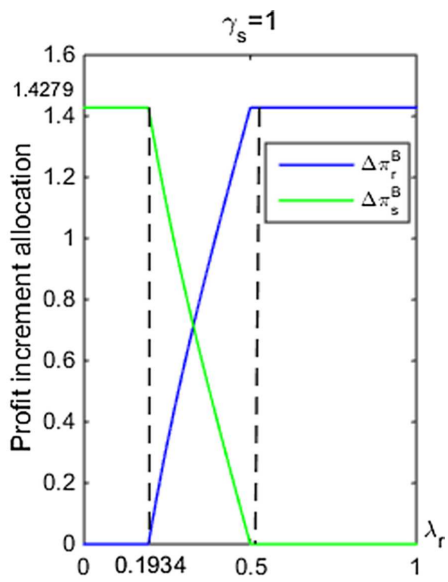

(2)

FIgURE 10. The optimal allocation of $\Delta \pi$ with $\lambda_{r}$.

would not be willing to cooperate with the risk-averse retailer. The retailer always tends to postpone order so that he can update demand information to reduce his overstock loss while correspondingly raise the supplier's overproduction loss. As a result, the risk-neutral supplier would be more resistant to play with the risk-averse retailer together, especially for the retailer who deliberately increases the degree of risk aversion.

Example 7.3. Figure 10 presents the impacts of the retailer's negotiation power on the optimal decisions and the additional supply chain expected profit allocation when only the supplier is risk-averse. From Figure 10, when $\gamma_{s}=1$, there are three cases: (1) if $0.5 \leq \lambda_{r} \leq 1$, then $\rho^{*}=1, \Delta \pi_{r}=1.4279$ and $\Delta \pi_{s}=0 ;(2)$ if $0.1934 \leq{ }_{r} \leq 0.5$, then $\rho^{*}=1-\frac{\ln \left(1-\lambda_{r}\right)-\ln \left(\lambda_{r}\right)}{2.0973}, \Delta \pi_{r}=1.4279-\frac{\ln \left(1-\lambda_{r}\right)}{\lambda_{r}}$ and $\Delta_{s}=\frac{\ln \left(1-\lambda_{r}\right)}{\lambda_{r}}$; and (3) if $0 \leq \lambda_{r} \leq 0.1934$, then $\rho^{*}=0.2879, \Delta \pi_{r}=0$ and $\Delta \pi_{s}=1.4279$. The risk-averse supplier with low negotiation power (e.g., $0 \leq \lambda_{s} \leq 0.5$ ), would not get additional profit from the centralized SC. As the supplier's negotiation power increases (e.g., $\left.0.5 \leq \lambda_{s} \leq 0.8066\right)$, she would obtain transfer payment of $\ln \frac{\lambda_{s}}{1-\lambda_{s}}$ from the retailer, which 


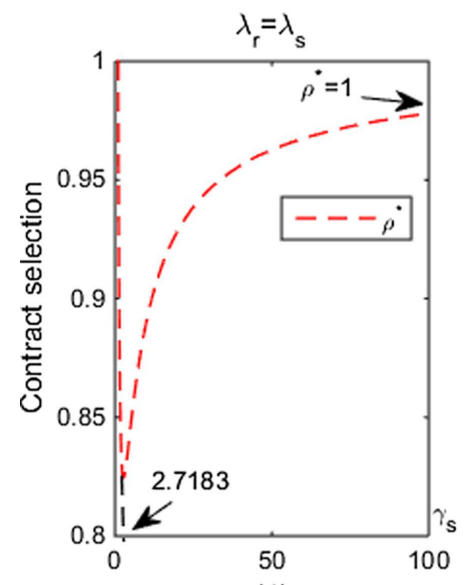

(1)

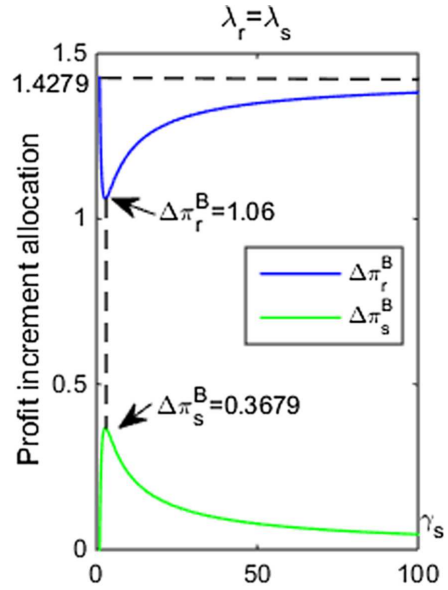

(2)

FIGURE 11. Contract selection and profit increment allocation with $\gamma_{s}$.

is increase with $\lambda_{s}$. Once the supplier's negotiation power is strong enough relative to the retailer's (e.g., $\left.\lambda_{s} \geq 0.8066\right)$, she would occupy the entire additional supply-chain profit.

Figure 11 shows the impact of the supplier's risk aversion on the optimal decisions and the additional supplychain expected profit allocation when both members have the same negotiation power. From Figure 11, when $\lambda_{r}=\lambda_{s}$ and if $\gamma_{s} \geq 1$, then $\rho^{*}=1-\frac{\ln \gamma_{s}}{2.0973 \gamma_{s}}, \Delta \pi_{r}=1.4279-\frac{\ln \gamma_{s}}{\gamma_{s}}$ and $\Delta \pi_{s}=\frac{\ln \gamma_{s}}{\gamma_{s}}$. In the case that the supplier is risk-averse and the retailer is risk-neutral, the supplier would not obtain additional expected profit from the centralized SC while only obtain transfer payment of $\frac{\ln \gamma_{s}}{\gamma_{s}}$ from the retailer, which is concave in $\gamma_{s}$. That suggests that the supplier with low risk aversion is beneficial (e.g., $\left.1 \leq \gamma_{s} \leq 2.7183\right)$. If the supplier raises risk aversion to a higher level (e.g., $\left.\gamma_{s} \geq 2.7183\right)$, he earns less and even not gain any transfer payment since the risk-neutral retailer may refuse to cooperate with the high risk-averse supplier.

\section{CONCLUSION AND MANAGEMENT INSIGHTS}

In this paper, we investigate the coordination issue of a two-echelon fresh product supply chain with demand information updating. By sharing the supply chain risks of both overproduction and overstock between the supplier and the retailer, we proposed a novel bi-directional risk-sharing contract (i.e., BRS contract) to coordinate such a supply chain. Our study shows that, compared with the single risk-sharing contracts (i.e., NRS, ORS-1 and ORS-2), any proposed contract in the set of $M$ can coordinate the supplier-retailer supply chain with Pareto-improvement. By adjusting risk-sharing proportions, the proposed contract can arbitrarily allocate the supply chain profit between the supplier and retailer. In addition, we further analyze how the supply chain members select an optimal bi-directional risk-sharing contract according to their risk preferences and negotiating powers. Our study shows that the extra profit each member could obtain from coordination increases with their relative power measurements while decreases with their relative risk aversion measurements.

The paper points out two main management insights. First, in the context of demand information updating, compared with sharing contract (i.e., NRS, ORS-1 and ORS-2 contracts), only the bi-directional risk-sharing contract can induce more production and ordering quantity, which leads greater supply chain profit. Hence, bi-directional risk-sharing between the supplier and retailer can be achieved on a voluntary compliance. Second, the risk aversion in some circumstances can improve supply chain members' profits and promote cooperation between the supplier and retailer. Hence, proper risk aversion measurements should be set to make cooperation 
relation a reality. Our finding demonstrates that the supply chain members only benefits from a relative lower risk aversion measurement.

The paper can be extended in the following two possible directions. First, in our model, we consider a twoechelon supply chain consisting of a single supplier and a single retailer. The retailer's final ordering quantity is constrained by the supplier's initial production quantity. In practice, the retailer, such as Toyota, would have multiple suppliers providing the same key components. Considering the supply chain with a retailer and multiple suppliers will yield interesting and meaningful results. Second, our current model only focuses on how the member's risk preferences affect their decision-making. In fact, the risk consideration is complex in the supply chain management, especially for the risk constraint supply chain members. As a natural extension of our work, future studies can consider coordination issues in the supply chain with demand information updating and risk constraint under the mean-variance framework.

\section{Appendix A.}

Proof of Proposition 4.1 and Corollary 4.2. At the beginning of Stage 2, given $Q_{s}^{\mathrm{SC}}$ and $I=i$, we get $\Pi^{\mathrm{SC}}\left(Q_{s}^{\mathrm{SC}}, i ; Q_{r}^{\mathrm{SC}}\right)=E_{\{x \mid i\}}\left\{p \min \left(x, Q_{r}^{\mathrm{SC}}\right)+v_{r}\left(Q_{r}^{\mathrm{SC}}-x\right)^{+}+v_{s}\left(Q_{s}^{\mathrm{SC}}-Q_{r}^{\mathrm{SC}}\right)\right\}=\left(p-v_{s}\right) Q_{r}^{\mathrm{SC}}-(p-$ $\left.v_{r}\right) \int_{0}^{Q_{r}^{\mathrm{SC}}} F(x \mid i) \mathrm{d} x+v_{s} Q_{s}^{\mathrm{SC}}$. Since $\frac{\partial \Pi^{\mathrm{SC}}\left(Q_{s}^{\mathrm{SC}}, i ; Q_{r}^{\mathrm{SC}}\right)}{\partial Q_{r}^{\mathrm{SC}}}=\left(p-v_{s}\right)-\left(p-v_{r}\right) F\left(Q_{r}^{\mathrm{SC}} \mid i\right)$ and $\frac{\partial^{2} \Pi^{\mathrm{SC}}\left(Q_{s}^{\mathrm{SC}}, i ; Q_{r}^{\mathrm{SC}}\right)}{\partial\left(Q_{r}^{\mathrm{SC}}\right)^{2}}=-(p-$ $\left.v_{r}\right) f\left(Q_{r}^{\mathrm{SC}} \mid i\right)<0, \Pi^{\mathrm{SC}}\left(Q_{s}^{\mathrm{SC}}, i ; Q_{r}^{\mathrm{SC}}\right)$ is concave in $Q_{r}^{\mathrm{SC}}$. Solving for first order condition, $Q_{r}^{\mathrm{SC}} \mid i=F^{-1}\left(\frac{p-v_{s}}{p-v_{r}}\right)$. Due to $Q_{r}^{\mathrm{SC}} \leq Q_{s}^{\mathrm{SC}}$, the optimal order quantity in centralized model is $Q_{r}^{\mathrm{SC}}=\min \left\{Q_{r}^{\mathrm{SC}} \mid i, Q_{s}^{\mathrm{SC}}\right\}$. Since $x \mid i$ is stochastically increasing in $i$, there is a positive threshold $i_{Q_{s}^{\mathrm{SC}}}$ that satisfies $Q_{r}^{\mathrm{SC}} \mid i_{Q_{s}^{\mathrm{SC}}}=Q_{s}^{\mathrm{SC}}$. Then, if $i \leq i_{Q_{s}^{\mathrm{SC}}}$, $Q_{r}^{\mathrm{SC}^{*}}=Q_{r}^{\mathrm{SC}} \mid i \leq Q_{s}^{\mathrm{SC}}$; if $i>i_{Q_{s}^{\mathrm{SC}}}, Q_{r}^{\mathrm{SC}}=Q_{s}^{\mathrm{SC}}<Q_{r}^{\mathrm{SC}} \mid i$. When market demand follows the uniform-uniform distribution, we have $F\left(Q_{r}^{\mathrm{SC}} \mid i\right)=\frac{1}{\beta}\left(Q_{r}^{\mathrm{SC}}-i+\frac{\beta}{2}\right)=\frac{p-v_{s}}{p-v_{r}}$. Then, we obtain $Q_{r}^{\mathrm{SC}} \mid i=i-\frac{\beta}{2}+\frac{p-v_{s}}{p-v_{r}} \beta$. Let $Q_{r}^{\mathrm{SC}} \mid i_{Q_{s}^{\mathrm{SC}}}=Q_{s}^{\mathrm{SC}}$, we have $i_{Q_{s}^{\mathrm{SC}}}=Q_{s}^{\mathrm{SC}}+\frac{\beta}{2}-\frac{p-v_{s}}{p-v_{r}} \beta$.

Proof of Proposition 4.3. At the beginning of Stage 1, since $\frac{\partial \Pi^{\mathrm{SC}}\left(Q_{s}^{\mathrm{SC}}, i ; Q_{r}^{\mathrm{SC}} \mid i\right)}{\partial Q_{s}^{\mathrm{SC}}}=v_{s}$ and $\frac{\partial \Pi^{\mathrm{SC}}\left(Q_{s}^{\mathrm{SC}}, i ; Q_{s}^{\mathrm{SC}}\right)}{\partial Q_{s}^{\mathrm{SC}}}=$ $\left[p-\left(p-v_{r}\right) F\left(Q_{s}^{\mathrm{SC}} \mid i\right)\right]$, then $\frac{\partial^{\mathrm{SC}}\left(Q_{s}^{\mathrm{SC}}\right)}{\partial Q_{s}^{\mathrm{SC}}}=\int_{0}^{i} Q_{s}^{\mathrm{SC}} \frac{\partial \Pi^{\mathrm{SC}}\left(Q_{s}^{\mathrm{SC}}, i ; Q_{r}^{\mathrm{SC}} \mid i\right)}{\partial Q_{s}^{\mathrm{SC}}} \mathrm{d} G(I)+\int_{i_{Q_{s}^{\mathrm{SC}}}}^{+\infty} \frac{\partial \Pi^{\mathrm{SC}}\left(Q_{s}^{\mathrm{SC}}, i ; Q_{s}^{\mathrm{SC}}\right)}{\partial Q_{s}^{\mathrm{SC}}} \mathrm{d} G(I)-c=v_{s}-$ $c+\int_{i_{S}^{\mathrm{SC}}}^{+\infty}\left[\left(p-v_{s}\right)-\left(p-v_{r}\right) F\left(Q_{s}^{\mathrm{SC}} \mid i\right)\right] \mathrm{d} G(I)$ and $\frac{\partial^{2} \Pi^{\mathrm{SC}}\left(Q_{s}^{\mathrm{SC}}\right)}{\partial\left(Q_{s}^{\mathrm{SC}}\right)^{2}}=-\int_{i_{Q_{s}^{S C}}}^{+\infty}\left[\left(p-v_{r}\right) f\left(Q_{s}^{\mathrm{SC}} \mid i\right)\right] \mathrm{d} G(I)<0$. Hence, $\Pi^{\mathrm{SC}}\left(Q_{s}^{\mathrm{SC}}\right)$ is concave in $Q_{s}^{\mathrm{SC}}$. Solving for first order condition, we have $\int_{i_{Q_{s} \mathrm{SC}}^{+\infty}}^{+\infty}\left[\frac{p-v_{s}}{p-v_{r}}-F\left(Q_{s}^{\mathrm{SC}} \mid i\right)\right] \mathrm{d} G(I)=\frac{c-v_{s}}{p-v_{r}}$. Note that, $F\left(Q_{r}^{\mathrm{SC}} \mid i\right)=\frac{p-v_{s}}{p-v_{r}}$. Hence, $Q_{s}^{\mathrm{SC}}$ is the unique solution of $\int_{i_{Q_{s}}^{\mathrm{SC}}}^{+\infty}\left[F\left(Q_{r}^{\mathrm{SC}} \mid i\right)-F\left(Q_{s}^{\mathrm{SC}} \mid i\right)\right] \mathrm{d} G(I)=$ $\frac{c-v_{s}}{p-v_{r}}$. In addition, when market demand follows the uniform-uniform distribution, we have $\left.\frac{1}{\alpha} \int_{Q_{s}^{\mathrm{SC}}+\frac{\beta}{2}-\frac{p-v_{s}}{p+v_{r}} \beta}^{\gamma+\frac{\alpha}{p-v_{r}}}-\frac{1}{\beta}\left(Q_{s}^{\mathrm{SC}}-i+\frac{\beta}{2}\right)\right] \mathrm{d} I=\frac{c-v_{s}}{p-v_{r}}$. Simplify, we have $\left(Q_{s}^{\mathrm{SC}}-\gamma-\frac{\alpha-\beta}{2}-\frac{p-v_{s}}{p-v_{r}} \beta\right)^{2}=$ $\frac{2\left(c-v_{s}\right) \alpha \beta}{p-v_{r}}$. Hence, $Q_{s}^{\mathrm{SC}^{*}}=\gamma+\frac{\alpha-\beta}{2}+\frac{p-v_{s}}{p-v_{r}} \beta-\sqrt{\frac{2\left(c-v_{s}\right) \alpha \beta}{p-v_{r}}}$.

Proof of Proposition 4.4 and Corollary 4.5. In the NRS model, at the beginning of Stage 2, given $Q_{s}^{N}$ and $I=i$, we get $\Pi_{r}^{N}\left(Q_{s}^{N}, i ; Q_{r}^{N}\right)=E_{\{x \mid i\}}\left\{p \min \left(x, Q_{r}^{N}\right)-w Q_{r}^{N}+v_{r}\left(Q_{r}^{N}-x\right)^{+}\right\}=(p-w) Q_{r}^{N}-\left(p-v_{r}\right) \int_{0}^{Q_{r}^{N}} F(x \mid i) \mathrm{d} x$. Since $\frac{\partial \Pi_{r}^{N}\left(Q_{s}^{N}, i ; Q_{r}^{N}\right)}{\partial Q_{r}^{N}}=(p-w)-\left(p-v_{r}\right) F\left(Q_{r}^{N} \mid i\right)$ and $\frac{\partial^{2} \Pi_{r}^{N}\left(Q_{s}^{N}, i ; Q_{r}^{N}\right)}{\partial\left(Q_{r}^{N}\right)^{2}}=-\left(p-v_{r}\right) f\left(Q_{r}^{N} \mid i\right)<0$, then $\Pi_{r}^{N}\left(Q_{s}^{N}, i ; Q_{r}^{N}\right)$ is concave in $Q_{r}^{N}$. Solving for first order condition, we have $Q_{r}^{N} \mid i=F^{-1}\left(\frac{p-w}{p-v_{r}}\right)$. Due to $Q_{r}^{N} \leq Q_{s}^{N}$, the optimal order quantity in the NRS model is $Q_{r}^{N^{*}}=\min \left\{Q_{r}^{N} \mid i, Q_{s}^{N}\right\}$. Since $x \mid i$ is stochastically increasing in $i$, there is a positive threshold $i_{Q_{s}^{N}}$ that satisfies $Q_{r}^{N} \mid i_{Q_{s}^{N}}=Q_{s}^{N}$. Then, if $i \leq i_{Q_{s}^{N}}, Q_{r}^{N^{*}}=Q_{r}^{N} \mid i \leq Q_{s}^{N}$; if $i>i_{Q_{s}^{N}}, Q_{r}^{N^{*}}=Q_{s}^{N}<Q_{r}^{N} \mid i$. In addition, when market demand follows the uniform-uniform distribution, we 
have $F\left(Q_{r}^{N} \mid i\right)=\frac{1}{\beta}\left(Q_{r}^{N}-i+\frac{\beta}{2}\right)=\frac{p-w}{p-v_{r}}$. Then, we obtain $Q_{r}^{N} \mid i=i-\frac{\beta}{2}+\frac{p-w}{p-v_{r}} \beta$. Let $Q_{r}^{N} \mid i_{Q_{s}^{N}}=Q_{s}^{N}$, we have $i_{Q_{s}^{N}}=Q_{s}^{N}+\frac{\beta}{2}-\frac{p-w}{p-v_{r}} \beta$.

Proof of Proposition 4.6. In the NRS model, since $\frac{\partial_{s}^{N}\left(Q_{s}^{N}, i ; Q_{r}^{N} \mid i\right)}{\partial Q_{s}^{N}}=v_{s}$ and $\frac{\partial \Pi_{s}^{N}\left(Q_{s}^{N}, i ; Q_{s}^{N}\right)}{\partial Q_{s}^{N}}=w$, then we have $\frac{\partial \Pi_{s}^{N}\left(Q_{s}^{N}\right)}{\partial Q_{s}^{N}}=\int_{0}^{i_{Q_{s}^{N}}} \frac{\partial_{s}^{N}\left(Q_{s}^{N}, i ; Q_{r}^{N} \mid i\right)}{\partial Q_{s}^{N}} \mathrm{~d} G(I)+\int_{i_{Q_{s}^{N}}^{+}}^{+\infty} \frac{\partial \Pi_{s}^{N}\left(Q_{s}^{N}, i ; Q_{s}^{N}\right)}{\partial Q_{s}^{N}} \mathrm{~d} G(I)-c=\int_{0}^{i_{Q_{s}}^{N}} v_{s} d G(I)+\int_{i_{Q_{s}^{N}}}^{+\infty} w d G(I)-c=$ $(w-c)-\left(w-v_{s}\right) G\left(i_{Q_{s}^{N}}\right)$ and $\frac{\partial^{2} \Pi_{s}^{N}\left(Q_{s}^{N}\right)}{\partial\left(Q_{s}^{N}\right)^{2}}=-\left(w-v_{s}\right) g\left(i_{Q_{s}^{N}}\right)<0$. Hence, $\Pi_{s}^{N}\left(Q_{s}^{N}\right)$ is concave in $Q_{s}^{N}$. Solving for first order condition, we have $G\left(i_{Q_{s}^{N}}\right)=\frac{w-c}{w-v_{s}}$. In addition, when market demand follows the uniformuniform distribution, we have $G\left(i_{Q_{s}^{N}}\right)=\frac{1}{\alpha}\left(i_{Q_{s}^{N}}-\gamma+\frac{\alpha}{2}\right)=\frac{w-c}{w-v_{s}}$. Thus, $i_{Q_{s}^{N}}=\gamma-\frac{\alpha}{2}+\frac{w-c}{w-v_{s}} \alpha$. Since $i_{Q_{s}^{N}}=$ $Q_{s}^{N}+\frac{\beta}{2}-\frac{p-w}{p-v_{r}} \beta$, we have $Q_{s}^{N^{*}}=\gamma+\frac{\alpha-\beta}{2}+\frac{p-w}{p-v_{r}} \beta-\frac{c-v_{s}}{w-v_{s}} \alpha$.

Proof of Proposition 4.7 and Corollary 4.8. In the ORS-1 model, at the beginning of Stage 2, given $Q_{s}^{S 1}$ and $I=i$, we have $\Pi_{r}^{S 1}\left(Q_{s}^{S 1}, i ; Q_{r}^{S 1}\right)=E_{\{x \mid i\}}\left\{p \min \left(x, Q_{r}^{S 1}\right)-w Q_{r}^{S 1}-\rho\left(c-v_{s}\right)\left(Q_{s}^{S 1}-Q_{r}^{S 1}\right)+v_{r}\left(Q_{r}^{S 1}-x\right)^{+}\right\}=(p-w+$ $\left.\rho\left(c-v_{s}\right)\right) Q_{r}^{S 1}-\left(p-v_{r}\right) \int_{0}^{Q_{r}^{S 1}} F(x \mid i) \mathrm{d} x-\rho\left(c-v_{s}\right) Q_{s}^{S 1}$. Since $\frac{\partial \Pi_{r}^{S 1}\left(Q_{s}^{S 1}, i ; Q_{r}^{S 1}\right)}{\partial ? Q_{r}^{S 1}}=\left(p-w+\rho\left(c-v_{s}\right)\right)-\left(p-v_{r}\right) F\left(Q_{r}^{S 1} \mid i\right)$ and $\frac{\partial^{2} \Pi_{r}^{S 1}\left(Q_{s}^{S 1}, i ; Q_{r}^{S 1}\right)}{\partial\left(Q_{r}^{S 1}\right)^{2}}=-\left(p-v_{r}\right) f\left(Q_{r}^{S 1} \mid i\right)<0$, then $\Pi_{r}^{S 1}\left(Q_{s}^{S 1}, i ; Q_{r}^{S 1}\right)$ is concave in $Q_{r}^{S 1}$. Solving for first order condition, we have $Q_{r}^{S 1} \mid i=F^{-1}\left(\frac{p-w+\rho\left(c-v_{s}\right)}{p-v_{r}}\right)$. Due to $Q_{r}^{S 1} \leq Q_{s}^{S 1}$, the optimal order quantity in the ORS model is $Q_{r}^{S 1^{*}}=\min \left\{Q_{r}^{S 1} \mid i, Q_{s}^{S 1}\right\}$. Since $x \mid i$ is stochastically increasing in $i$, there is a positive threshold $i_{Q_{s}^{S 1}}$ that satisfies $Q_{r}^{S 1} \mid i_{Q_{s}^{S 1}}=Q_{s}^{S 1}$. Then, if $i \leq i_{Q_{s}^{S 1}}, Q_{r}^{S 1^{*}}=Q_{r}^{S 1} \mid i \leq Q_{s}^{S 1}$; if $i>i_{Q_{s}^{S 1}}, Q_{r}^{S 1^{*}}=Q_{s}^{S 1}<Q_{r}^{S 1} \mid i$. When market demand is uniform-uniform distributed, $F\left(Q_{r}^{S 1} \mid i\right)=\frac{1}{\beta}\left(Q_{r}^{S 1}-i+\frac{\beta}{2}\right)=\frac{p-w+\rho\left(c-v_{s}\right)}{p-v_{r}}$. Then, $Q_{r}^{S 1} \mid i=i-\frac{\beta}{2}+\frac{p-w+\rho\left(c-v_{s}\right)}{p-v_{r}} \beta$. Let $Q_{r}^{S 1} \mid i_{Q_{s}^{S 1}}=Q_{s}^{S 1}$, where $i_{Q_{s}^{S 1}}=Q_{s}^{S 1}+\frac{\beta}{2}-\frac{p-w+\rho\left(c-v_{s}\right)}{p-v_{r}} \beta$.

Proof of Proposition 4.9. In the ORS model, at the beginning of Stage 1, since $\frac{\partial \Pi_{s}^{S 1}\left(Q_{s}^{S 1}, i ; Q_{r}^{S 1} \mid i\right)}{\partial Q_{s}^{S 1}}=v_{s}+\rho\left(c-v_{s}\right)$ and $\frac{\partial \Pi_{s}^{S 1}\left(Q_{s}^{S 1}, i ; Q_{s}^{S 1}\right)}{\partial Q_{s}^{S 1}}=w$, then we have $\frac{\partial \Pi_{s}^{S 1}\left(Q_{s}^{S 1}\right)}{\partial Q_{s}^{S 1}}=\int_{0}^{i_{s}^{S 1}} \frac{\partial \Pi_{s}^{S 1}\left(Q_{s}^{S 1}, i ; Q_{r}^{S 1} \mid i\right)}{\partial Q_{s}^{S 1}} \mathrm{~d} G(I)+\int_{i_{Q_{s}^{S 1}}}^{+\infty} \frac{\partial \Pi_{s}^{S 1}\left(Q_{s}^{S 1}, i ; Q_{s}^{S 1}\right)}{\partial Q_{s}^{S 1}} \mathrm{~d} G(I)-$ $c=\int_{0}^{i} Q_{s}^{S 1}\left[v_{s}+\rho\left(c-v_{s}\right)\right] \mathrm{d} G(I)+\int_{i_{Q_{s}^{S 1}}}^{+\infty} w d G(I)-c=(w-c)-\left(w-v_{s}-\rho\left(c-v_{s}\right)\right) G\left(i_{Q_{s}^{S 1}}\right)$ and $\frac{\partial^{2} \Pi_{s}^{S 1}\left(Q_{s}^{S 1}\right)}{\partial\left(Q_{s}^{S 1}\right)^{2}}=$ $-\left(w-v_{s}-\rho\left(c-v_{s}\right)\right) g\left(i_{Q_{s}^{S 1}}\right)<0$. Hence, $\Pi_{s}^{S 1}\left(Q_{s}^{S 1}\right)$ is concave in $Q_{s}^{S 1}$. Solving for first order condition, we have $G\left(i_{Q_{s}^{S 1}}\right)=\frac{w-c}{w-v_{s}-\rho\left(c-v_{s}\right)}$. In addition, when market demand follows the uniform-uniform distribution, we have $G\left(i_{Q_{s}^{S 1}}\right)=\frac{1}{\alpha}\left(i_{Q_{s}^{S 1}}-\gamma+\frac{\alpha}{2}\right)=\frac{w-c}{w-v_{s}-\rho\left(c-v_{s}\right)}$. Thus, $i_{Q_{s}^{S 1}}=\gamma-\frac{\alpha}{2}+\frac{w-c}{w-v_{s}-\rho\left(c-v_{s}\right)} \alpha$. Since $i_{Q_{s}^{S 1}}=$ $Q_{s}^{S 1}+\frac{\beta}{2}-\frac{p-w+\rho\left(c-v_{s}\right)}{p-v_{r}} \beta$, then $Q_{s}^{S 1^{*}}=\gamma+\frac{\alpha-\beta}{2}+\frac{p-w+\rho\left(c-v_{s}\right)}{p-v_{r}} \beta-\frac{(1-\rho)\left(c-v_{s}\right)}{w-v_{s}-\rho\left(c-v_{s}\right)} \alpha$.

Proof of Proposition 4.10 and Corollary 4.11. In the ORS-2 model, at the beginning of Stage 2, given $Q_{s}^{S 2}$ and $I=i, \Pi^{S 2}\left(Q_{s}^{S 2}, i ; Q_{r}^{S 2}\right)=E_{\{x \mid i\}}\left\{p \min \left(x, Q_{r}^{S 2}\right)-w Q_{r}^{S 2}+\left[v_{r}+\phi\left(w-v_{r}\right)\right]\left(Q_{r}^{S 2}-x\right)^{+}\right\}=(p-w) Q_{r}^{S 2}-$ $\left(p-v_{r}-\phi\left(w-v_{r}\right)\right) \int_{0}^{Q_{r}^{S 2}} F(x \mid i) \mathrm{d} x$. Since $\frac{\partial \Pi^{S 2}\left(Q_{s}^{S 2}, i ; Q_{r}^{S 2}\right)}{\partial Q_{r}^{S 2}}=(p-w)-\left(p-v_{r}-\phi\left(w-v_{r}\right)\right) F\left(Q_{r}^{S 2} \mid i\right)$ and $\frac{\partial^{2 S 2}\left(Q_{s}^{S 2}, i ; Q_{r}^{S 2}\right)}{\partial\left(Q_{r}^{S 2}\right)^{2}}=-\left(p-v_{r}-\phi\left(w-v_{r}\right)\right) f\left(Q_{r}^{S 2} \mid i\right)<0$, then $\Pi^{S 2}\left(Q_{s}^{S 2}, i ; Q_{r}^{S 2}\right)$ is concave in $Q_{r}^{S 2}$. Solving for first order condition, we have $Q_{r}^{S 2} \mid i=F^{-1}\left(\frac{p-w}{p-v_{r}-\phi\left(w-v_{r}\right)}\right)$. Due to $Q_{r}^{S 2} \leq Q_{s}^{S 2}$, the optimal order quantity in centralized model is $Q_{r}^{S 2^{*}}=\min \left\{Q_{r}^{S 2} \mid i, Q_{s}^{S 2}\right\}$. Since $x \mid i$ is stochastically increasing in $i$, there exists a unique positive threshold $i_{Q_{s}^{S 2}}$ that satisfies $Q_{r}^{S 2} \mid i_{Q_{s}^{S 2}}=Q_{s}^{S 2}$. Then, if $i \leq i_{Q_{s}^{S 2}}, Q_{r}^{S 2^{*}}=Q_{r}^{S 2} \mid i \leq Q_{s}^{S 2}$; if $i>i_{Q_{s}^{S 2}}, Q_{r}^{S 2^{*}}=Q_{s}^{S 2}<Q_{r}^{S 2} \mid i$. In addition, when market demand follows the uniform-uniform distribution, we have $F\left(Q_{r}^{S 2} \mid i\right)=\frac{1}{\beta}\left(Q_{r}^{S 2}-i+\frac{\beta}{2}\right)=\frac{p-w}{p-v_{r}-\phi\left(w-v_{r}\right)}$. Then, we obtain $Q_{r}^{S 2} \mid i=i-\frac{\beta}{2}+\frac{p-w}{p-v_{r}-\phi\left(w-v_{r}\right)} \beta$. Let $Q_{r}^{S 2} \mid i_{Q_{s}^{S 2}}=Q_{s}^{S 2}$, we have $i_{Q_{s}^{S 2}}=Q_{s}^{S 2}+\frac{\beta}{2}-\frac{p-w}{p-v_{r}-\phi\left(w-v_{r}\right)} \beta$. 
Proof of Proposition 4.12. In the ORS-2 model, at the beginning of Stage 1, since $\frac{\partial \Pi_{s}^{S 2}\left(Q_{s}^{S 2}, i ; Q_{r}^{S 2} \mid i\right)}{\partial Q_{s}^{S 2}}=$ $v_{s}$ and $\frac{\partial \Pi_{s}^{S 2}\left(Q_{s}^{S 2}, i ; Q_{s}^{S 2}\right)}{\partial Q_{s}^{S 2}}=\left[w-\phi\left(w-v_{r}\right) F\left(Q_{s}^{S 2} \mid i\right)\right]$, then $\frac{\Pi_{s}^{S 2}\left(Q_{s}^{S 2}\right)}{\partial Q_{s}^{S 2}}=\int_{0}^{i} Q_{s}^{S 2} \frac{\partial \Pi_{s}^{S 2}\left(Q_{s}^{S 2}, i ; Q_{r}^{S 2} \mid i\right)}{\partial Q_{s}^{S 2}} \mathrm{~d} G(I)+$ $\int_{i_{Q}^{S 2}}^{+\infty} \frac{\partial \Pi_{s}^{S 2}\left(Q_{s}^{S 2}, i Q_{s}^{S 2}\right)}{\partial Q_{s}^{S 2}} \mathrm{~d} G(I)-c=\int_{0}^{i_{Q} Q_{s}^{S 2}} v_{s} d G(I)+\int_{i_{Q_{s}^{S 2}}}^{+\infty}\left[w-\phi\left(w-v_{r}\right) F\left(Q_{s}^{S 2} \mid i\right)\right] \mathrm{d} G(I)-c=v_{s}-$ $c+\int_{i_{Q_{s}^{S}}}^{+\infty}\left[\left(w-v_{s}\right)-\phi\left(w-v_{r}\right) F\left(Q_{s}^{S 2} \mid i\right)\right] \mathrm{d} G(I)$ and $\frac{\partial^{2} \Pi_{s}^{S 2}\left(Q_{s}^{S 2}\right)}{\partial\left(Q_{s}^{S 2}\right)^{2}}=-\int_{i_{Q_{s}^{S 2}}}^{+\infty} \phi\left(w-v_{r}\right) f\left(Q_{s}^{S 2} \mid i\right) \mathrm{d} G(I)<$ 0 . Hence, $\Pi_{s}^{S 2}\left(Q_{s}^{S 2}\right.$ is concave in $Q_{s}^{S 2}$. Solving for first order condition, we have $\int_{i_{Q S}^{S 2}}^{+\infty}\left[\frac{w-v_{s}}{\left(w-v_{r}\right)}-\right.$ $\left.F\left(Q_{s}^{S 2} \mid i\right)\right] \mathrm{d} G(I)=\frac{c-v_{s}}{\phi\left(w-v_{r}\right)}$. In addition, when market demand follows the uniform-uniform distribution, we have $\frac{1}{\alpha} \int_{Q_{s}^{S 2}+\frac{\beta}{2}-\frac{p}{p-v_{r}-\phi\left(w-v_{r}\right)} \beta}^{\gamma+\frac{\alpha}{\phi}}\left[\frac{w-v_{s}}{\phi\left(w-v_{r}\right)}-\frac{1}{\beta}\left(Q_{s}^{S 2}-i+\frac{\beta}{2}\right)\right] \mathrm{d} I=\frac{c-v_{s}}{\phi\left(w-v_{r}\right)}$. Simplify, we have $\left(Q_{s}^{S 2}-\gamma-\frac{\alpha-\beta}{2}-\frac{w-v_{s}}{\phi\left(w-v_{r}\right)} \beta\right)^{2}=\left[\frac{w-v_{s}}{\phi\left(w-v_{r}\right)} \beta-\frac{p-w}{p-v_{r}-\phi\left(w-v_{r}\right)} \beta\right]^{2}+\frac{2\left(c-v_{s}\right) \alpha \beta}{\phi\left(w-v_{r}\right)}$. Hence, $Q_{s}^{S 2^{*}}=\gamma+\frac{\alpha-\beta}{2}+$ $\frac{w-v_{s}}{\phi\left(w-v_{r}\right)} \beta-\sqrt{\left[\frac{w-v_{s}}{\phi\left(w-v_{r}\right)} \beta-\frac{p-w}{p-v_{r}-\phi\left(w-v_{r}\right)} \beta\right]^{2}+\frac{2\left(c-v_{s}\right) \alpha \beta}{\phi\left(w-v_{r}\right)}}$.

Proof of Proposition 4.13 and Corollary 4.14. In the BRS model, at the beginning of Stage 2, given $Q_{s}^{B}$ and $I=i, \Pi^{B}\left(Q_{s}^{B}, i ; Q_{r}^{B}\right)=\Pi_{r}^{B}\left(Q_{s}^{B}, i ; Q_{r}^{B}\right)=E_{\{x \mid i\}}\left\{p \min x, Q_{r}^{B}-w Q_{r}^{B}+\left[\phi\left(w-v_{r}\right)+v_{r}\right]\left(Q_{r}^{B}-x\right)^{+}-\rho(c-\right.$ $\left.\left.v_{s}\right)\left(Q_{s}^{B}-Q_{r}^{B}\right)\right\}=\left[(p-w)+\rho\left(c-v_{s}\right)\right] Q_{r}^{B}-\left[p-v_{r}-\phi\left(w-v_{r}\right)\right] \int_{0}^{Q_{r}^{B}} F(x \mid i) \mathrm{d} x$. Since $\frac{\partial \Pi^{B}\left(Q_{s}^{B}, i ; Q_{r}^{B}\right)}{\partial Q_{r}^{B}}=[(p-w)+$ $\left.\rho\left(c-v_{s}\right)\right]-\left[p-v_{r}-\phi\left(w-v_{r}\right)\right] F\left(Q_{r}^{B} \mid i\right)$ and $\frac{\partial^{2} \Pi^{B}\left(Q_{s}^{B}, i ; Q_{r}^{B}\right)}{\partial\left(Q_{r}^{B}\right)^{2}}=-\left[p-v_{r}-\phi\left(w-v_{r}\right)\right] f\left(Q_{r}^{B} \mid i\right)<0, \Pi^{B}\left(Q_{s}^{B}, i ; Q_{r}^{B}\right)$ is concave in $Q_{r}^{B}$. Solving for first order condition, we have $Q_{r}^{B} \mid i=F^{-1} \frac{p-w+\rho\left(c-v_{s}\right)}{p-v_{r}-\phi\left(w-v_{r}\right)}$. Due to $Q_{r}^{B} \leq Q_{s}^{B}$, the optimal order quantity in centralized model is $Q_{r}^{B^{*}}=\left\{Q_{r}^{B} \mid i, Q_{s}^{B}\right\}$. Since $x \mid i$ is stochastically increasing in $i$, there exists a unique positive threshold $i_{Q_{s}^{B}}$ that satisfies $Q_{r}^{B} \mid i_{Q_{s}^{B}}=Q_{s}^{B}$. Then, if $i \leq i_{Q_{s}^{B}}, Q_{r}^{B^{*}}=Q_{r}^{B} \mid i \leq Q_{s}^{B}$; if $i>i_{Q_{s}^{B}}, Q_{r}^{B^{*}}=Q_{s}^{B}<Q_{r}^{B} \mid i$. In addition, when market demand follows the uniform-uniform distribution, we have $F\left(Q_{r}^{B} \mid i\right)=\frac{1}{\beta}\left(Q_{r}^{B}-i+\frac{\beta}{2}\right)=\frac{p-w+\rho\left(c-v_{s}\right)}{p-v_{r}-\phi\left(w-v_{r}\right)}$. Then, we obtain $Q_{r}^{B} \mid i=i-\frac{\beta}{2}+\frac{p-w+\rho\left(c-v_{s}\right)}{p-v_{r}-\phi\left(w-v_{r}\right)} \beta$. Let $Q_{r}^{B} \mid i_{Q_{s}^{B}}=Q_{s}^{B}$, we have $i_{Q_{s}^{B}}=Q_{s}^{B}+\frac{\beta}{2}-\frac{p-w+\rho\left(c-v_{s}\right)}{p-v_{r}-\phi\left(w-v_{r}\right)} \beta$.

Proof of Proposition 4.15. In the BRS model, at the beginning of Stage 1, since $\frac{\partial \Pi_{s}^{B}\left(Q_{s}^{B}, i, Q_{r}^{B} \mid i\right)}{\partial Q_{s}^{B}}=v_{s}+$ $\rho\left(c-v_{s}\right)$ and $\frac{\partial \Pi_{s}^{B}\left(Q_{s}^{B}, i ; Q_{s}^{B}\right)}{\partial Q_{s}^{B}}=\left[w-\phi\left(w-v_{r}\right) F\left(Q_{s}^{B} \mid i\right)\right]$, then $\frac{\partial \Pi_{s}^{B}\left(Q_{s}^{B}\right)}{\partial Q_{s}^{B}}=\int_{0}^{i} Q_{s}^{B} \frac{\partial \Pi_{s}^{B}\left(Q_{s}^{B}, i ; Q_{r}^{B} \mid i\right)}{\partial Q_{s}^{B}} \mathrm{~d} G(I)+$ $\int_{i_{Q_{s}^{B}}^{+}}^{+\infty} \frac{\partial \Pi_{s}^{B}\left(Q_{s}^{B}, i ; Q_{s}^{B}\right)}{\partial Q_{s}^{B}} \mathrm{~d} G(I)-c=\int_{0}^{i_{Q_{s}^{B}}^{B}}\left[v_{s}+\rho\left(c-v_{s}\right)\right] \mathrm{d} G(I)+\int_{i_{Q_{s}^{B}}}^{+\infty}\left[w-\phi\left(w-v_{r}\right) F\left(Q_{s}^{B} \mid i\right)\right] \mathrm{d} G(I)-c=$ $(1-\rho)\left(v_{s}-c\right)+\int_{i_{Q_{s}^{B}}^{B}}^{+\infty}\left[\left[w-v_{s}-\rho\left(c-v_{s}\right)\right]-\phi\left(w-v_{r}\right) F\left(Q_{s}^{B} \mid i\right)\right] \mathrm{d} G(I)$ and $\frac{\partial^{2} \Pi_{s}^{B}\left(Q_{s}^{B}\right)}{\partial\left(Q_{s}^{B}\right)^{2}}=-\int_{i_{Q_{s}^{B}}}^{+\infty} \phi(w-$ $\left.v_{r}\right) f\left(Q_{s}^{B} \mid i\right) \mathrm{d} G(I)<0$. Hence, $\Pi_{s}^{B}\left(Q_{s}^{B}\right)$ is concave in $Q_{s}^{B}$. Solving for first order condition, we have $\int_{i_{Q_{s}^{B}}^{+}}^{+\infty}\left[\frac{w-v_{s}-\rho\left(c-v_{s}\right)}{\phi\left(w-v_{r}\right)}-F\left(Q_{s}^{B} i\right)\right] \mathrm{d} G(i)=\frac{(1-\rho)\left(c-v_{s}\right)}{\phi\left(w-v_{r}\right)}$. When market demand follows the uniform-uniform distribution, we have $\frac{1}{\alpha} \int_{Q_{s}^{B}+\frac{\beta}{2}-\frac{p-w}{p-v_{r}-\phi\left(w-v_{r}\right)}}^{\gamma+\frac{\alpha}{2}}\left[\frac{w-v_{s}-\rho\left(c-v_{s}\right)}{\phi\left(w-v_{r}\right)} \beta-\frac{1}{\beta}\left(Q_{s}^{B}-i+\frac{\beta}{2}\right)\right] \mathrm{d} I=\frac{(1-\rho)\left(c-v_{s}\right)}{\phi\left(w-v_{r}\right)}$, then $\left(Q_{s}^{B}-\gamma-\frac{\alpha-\beta}{2}-\frac{w-v_{s}-\rho\left(c-v_{s}\right)}{\phi\left(w-v_{r}\right)} \beta\right)^{2}=\left[\frac{w-v_{s}-\rho\left(c-v_{s}\right)}{\phi\left(w-v_{r}\right)} \beta-\frac{p-w}{p-v_{r}-\phi\left(w-v_{r}\right)} \beta\right]^{2}+\frac{2(1-\rho)\left(c-v_{s}\right) \alpha \beta}{\phi\left(w-v_{r}\right)}$, and $Q_{s}^{B^{*}}=\gamma+$ $\frac{\alpha-\beta}{2}+\frac{w-v_{s}-\rho\left(c-v_{s}\right)}{\phi\left(w-v_{r}\right)} \beta-\sqrt{\left[\frac{w-v_{s}-\rho\left(c-v_{s}\right)}{\phi\left(w-v_{r}\right)} \beta-\frac{p-w}{p-v_{r}-\phi\left(w-v_{r}\right)} \beta\right]^{2}+\frac{2(1-\rho)\left(c-v_{s}\right) \alpha \beta}{\phi\left(w-v_{r}\right)}}$.

Proof of Propositions 5.1 and 5.2. Let $Q_{r}^{B}\left|i=Q_{r}^{c}\right| i$, then $w=p+\rho\left(c-v_{s}\right)-\frac{p-v_{s}}{p-v_{r}}\left[p-\phi\left(w-v_{r}\right)-v_{r}\right]$. Inserting this $w$ into equation (4.7), an equivalent form of equation (4.7) is $\int_{i_{Q_{s}^{B}}}^{+\infty}\left[F\left(Q_{r}^{B} i\right)-F\left(Q_{r}^{\mathrm{SC}} \mid i\right)\right] \mathrm{d} G(i)=\frac{(1-)\left(c-v_{s}\right)}{\phi\left(w-v_{r}\right)}$. Compared with equation (4.4), as long as $1-\rho=\frac{w-v_{r}}{p-v_{r}} \phi$, equations (4.4) and (4.7) are equivalent, i.e., $Q_{s}^{\mathrm{SC}^{*}}=$ $Q_{s}^{B^{*}}$. Hence, when $w=p-\rho(p-c),(1-\rho)\left(p-v_{r}\right)=\phi\left(w-v_{r}\right)$, the optimal decisions in the decentralized 
system will consist with its counterpart in the centralized system, i.e., the supply chain coordination can be achieved.

Proof of Lemma 6.2. Since $u^{1}=-\lambda_{r} \exp ^{-\gamma_{r}\left[\rho \pi-\pi_{r}^{N}\right]}-\lambda_{s} \exp ^{-\gamma_{s}\left[(1-\rho) \pi-\pi_{s}^{N}\right]}$, then we have $\frac{\partial u^{1}}{\partial \rho}=$ $\lambda_{r} \gamma_{r} \pi \exp ^{-\gamma_{r}\left[\rho \pi-\pi^{N}\right]}-\lambda_{s} \gamma_{s} \pi \exp ^{-\gamma_{s}\left[(1-\rho) \pi-\pi^{N}\right]}$, and $\frac{\partial^{2} u^{1}}{\partial \rho^{2}}=-\lambda_{r}\left(\gamma_{r} \pi\right)^{2} \exp ^{-\gamma_{r}\left[\rho \pi-\pi^{N}\right]}$

$-\lambda_{s}\left(\gamma_{s} \pi\right)^{2} \exp ^{-\gamma_{s}\left[(1-\rho) \pi-\pi^{N}\right]}<0$, thus $u^{1}$ is concave in $\rho$. Let $\frac{\partial u^{1}}{\partial \rho}=0$, we have optimal $\bar{\rho}_{1}=\frac{\gamma_{r}}{\gamma_{r}+\gamma_{s}} \rho_{\min }+$ $\frac{\gamma_{s}}{\gamma_{r}+\gamma_{s}} \rho_{\max }-\frac{\ln \frac{\lambda_{s} \gamma_{s}}{\lambda_{r} \gamma_{r}}}{\left.\gamma_{r}+\gamma_{s}\right) \pi}$. Since $\rho \in\left[\rho_{\min }, \rho_{\max }\right]$, if $\bar{\rho}_{1} \geq \rho_{\max }$, i.e., $\frac{\lambda_{s}}{\lambda_{r}} \leq \frac{\gamma_{r}}{\gamma_{s}} \exp ^{-\gamma_{r} \pi}$, then $\rho^{*}=\rho_{\max }, \Delta \pi_{r}=\Delta \pi$ and $\Lambda \pi_{s}=0$; If $\bar{\rho}_{1} \in\left[\rho_{\min }, \rho_{\max }\right]$, i.e., $\frac{\gamma_{r}}{\gamma_{s}} \exp ^{-\gamma_{r} \Delta \pi} \leq \frac{\lambda_{s}}{\lambda_{r}} \leq \frac{\gamma_{r}}{\gamma_{s}} \exp ^{\gamma_{s} \Delta \pi}$, then $\rho^{*}=\frac{\gamma_{r}}{\gamma_{r}+\gamma_{s}} \rho_{\min }+\frac{\gamma_{s}}{\gamma_{r}+\gamma_{s}} \rho_{\max }-$ $\frac{\ln \lambda_{s} \gamma_{s} \lambda_{r} \gamma_{r}}{\left(\gamma_{r}+\gamma_{s}\right) \pi}, \Delta \pi_{r}=\frac{\gamma_{s}}{\gamma_{r}+\gamma_{s}} \Delta \pi-\frac{\ln \frac{\lambda_{s} \gamma_{s}}{\lambda_{r} \gamma_{r}}}{\gamma_{r}+\gamma_{s}}$ and $\Delta \pi_{s}=\frac{\gamma_{r}}{\gamma_{r}+\gamma_{s}} \Delta \pi+\frac{\ln \frac{\lambda_{s} \gamma_{s}}{\lambda_{r} \gamma_{r}}}{\gamma_{r}+\gamma_{s}}$. If $\bar{\rho}_{1} \leq \rho_{\min }$, i.e., $\frac{\lambda_{s}}{\lambda_{r}} \geq \frac{\gamma_{r}}{\gamma_{s}} \exp ^{\gamma_{s} \Delta \pi}$, then $\rho^{*} \stackrel{\gamma_{r}+\gamma_{s}}{=} \rho_{\min }, \Delta \pi_{r}=0$ and $\Delta \pi_{s}=\Delta \pi$.

Proof of Lemma 6.4. Since $u^{2}=-\lambda_{r} \exp ^{-\gamma_{r} \Delta \pi_{r}}+\lambda_{s} \Delta \pi_{s}=-\lambda_{r} \exp ^{-\gamma_{r}\left[\rho \pi-\pi_{r}^{N}\right]}+\lambda_{s}\left[(1-\rho) \pi-\pi_{s}\right]$, then $\frac{\partial u^{2}}{\partial \rho}=$ $\lambda_{r} \gamma_{r} \pi \exp ^{-\gamma_{r}\left[\rho \pi-\pi_{r}^{N}\right]}-\lambda_{s} \pi, \frac{\partial^{2} u^{2}}{\partial \rho^{2}}=-\lambda_{r}\left(\gamma_{r} \pi\right)^{2} \exp ^{-\gamma_{r}\left[\rho \pi-\pi_{r}^{N}\right]}<0$, hence $u^{2}$ is concave in $\rho$. Let $\frac{\partial u^{2}}{\partial \rho}=0$, we have optimal $\bar{\rho}_{2}=\rho_{\min }+\frac{\ln \frac{\lambda_{r} \gamma_{r}}{\lambda_{s}}}{\gamma_{r} \pi}$. Since $\rho \in\left[\rho_{\min }, \rho_{\max }\right]$, if $\bar{\rho}_{2} \geq \rho_{\max }$, i.e., $\frac{\lambda_{r}}{\lambda_{s}} \geq \exp ^{\gamma_{r} \Delta \pi} \gamma_{r}$, then $\rho^{*}=\rho_{\max }$, $\Delta \pi_{r}=\Delta \pi$ and $\Delta \pi_{s}=0 ;$ If $\bar{\rho}_{2} \in\left[\rho_{\min }, \rho_{\max }\right]$, i.e., $\frac{1}{\gamma_{r}}<\frac{\lambda_{r}}{\lambda_{s}}<\exp ^{\gamma_{r} \Delta \pi} \gamma_{r}$, then $\rho^{*}=\rho_{\min }+\frac{\ln \frac{\lambda_{r} \gamma_{r}}{\lambda_{s}}}{\gamma_{r} \pi}, \Delta \pi_{r}=\frac{\ln \frac{\lambda_{r} \gamma_{r}}{\lambda_{s}}}{\gamma_{r}}$ and $\Delta \pi_{s}=\Delta \pi-\frac{\ln \frac{\lambda_{r} \gamma_{r}}{\lambda_{s}}}{\gamma_{r}}$; If $\bar{\rho}_{2} \leq \rho_{\min }$, i.e., $\frac{\lambda_{r}}{\lambda_{s}} \leq \frac{1}{\gamma_{r}}$, then $\rho^{*}=\rho_{\min }, \Delta \pi_{r}=0$ and $\Delta \pi_{s}=\Delta \pi$.

Proof of Lemma 6.6. Since $u^{3}=\lambda_{r}\left[\rho \pi-\pi_{r}^{N}\right]-\lambda_{s} \gamma_{s} \pi \exp ^{-\gamma_{s}\left[(1-\rho) \pi-\pi_{s}^{N}\right]}$, then $\frac{\partial u^{3}}{\partial \rho}=\lambda_{r} \pi-$ $\lambda_{s} \gamma_{s} \pi \exp ^{-\gamma_{s}\left[(1-\rho) \pi-\pi_{s}^{N}\right]}$, and $\frac{\partial^{2} u^{3}}{\partial \rho^{2}}=-\lambda_{s}\left(\gamma_{s} \pi\right)^{2} \exp ^{-\gamma_{s}\left[(1-\rho) \pi-\pi_{s}^{N}\right]}<0$, hence $u^{3}$ is concave in $\rho$. Let $\frac{\partial u^{3}}{\partial \rho}=0$, we have optimal $\bar{\rho}_{3}=\rho_{\max }-\frac{\ln \frac{\lambda_{s} \nu_{s}}{\lambda_{r}}}{\gamma_{s} \pi}$. Since $\rho \in\left[\rho_{\min }, \rho_{\max }\right]$, if $\bar{\rho}_{3} \geq \rho_{\max }$, i.e., $\frac{\lambda_{s}}{\lambda_{r}} \leq \frac{1}{\gamma_{s}}$, then $\rho^{*}=\rho_{\max }, \Delta \pi_{r}^{B}=\Delta \pi$ and $\Delta \pi_{s}^{B}=0$; If $\bar{\rho}_{3} \in\left[\rho_{\min }, \rho_{\max }\right]$, i.e., $\frac{1}{\gamma_{s}} \leq \frac{\lambda_{s}}{\lambda_{r}} \leq \frac{1}{\gamma_{s}} \exp ^{\gamma_{s} \Delta \pi}$, then $\rho^{*}=\rho_{\max }-\frac{\ln \frac{\lambda_{s} \gamma_{s}}{\lambda_{r}}}{\gamma_{s} \pi}, \Delta \pi_{r}=\Delta \pi-\frac{\ln \frac{\lambda_{s} \gamma_{s}}{\lambda_{r}}}{\gamma_{s}}$ and $\Delta \pi_{s}=\frac{\ln \frac{\lambda_{s} \gamma_{s}}{\lambda_{r}}}{\gamma_{s}}$; If $\bar{\rho}_{3} \leq \rho_{\min }$, i.e., $\frac{\lambda_{s}}{\lambda_{r}} \geq \frac{1}{\gamma_{s}} \exp \gamma_{s} \Delta \pi$, then $\rho^{*}=\rho_{\min }, \Delta \pi_{r}=0$ and $\Delta \pi_{s}=\Delta \pi$.

Acknowledgements. The authors would like to thank the editor and the anonymous referees for their helpful comments and suggestions that greatly improved the quality of this paper. The research is supported by the National Natural Science Foundation of China under Grant Nos. 72071072 and the Postgraduate Scientific Research Innovation Project of Hunan Province under Grant Nos. CX20200456.

\section{REFERENCES}

[1] A. Adhikari, A. Bisi and B. Avittathur, Coordination mechanism, risk sharing, and risk aversion a five-level textile supply chain under demand and supply uncertainty. Eur. J. Oper. Res. 282 (2020) 93-107.

[2] A. Ahmadi-Javid and P. Hoseinpour, A location inventory pricing model in a supply chain distribution network with price sensitive demands and inventory capacity constraints. Transp. Res. Part E: Logistics Transp. Rev. 82 (2015) $238-255$.

[3] H.V. Arani, M. Rabbani and H. Rafiei, A revenue-sharing option contract toward coordination of supply chains. Int. J. Prod. Econ. 178 (2016) 42-56.

[4] B. Barnes-Schuster, Y. Bassok and R. Anupindi, Coordination and flexibility in supply contracts with options. Manuf. Serv. Oper. Manage. 4 (2002) 171-240.

[5] G. Cachon, Supply chain coordination with contracts, chapter 6, edited by S. Graves and T. de Kok. In: Handbooks in Operations Research and Management Science: Supply Chain Management. North Holland (2003) 227-339.

[6] G. Cachon and R. Swinney, Purchasing, pricing and quick response in the presence of strategic consumers. Manage. Sci. 55 (2009) 497-511.

[7] G. Cachon and R. Swinney, The value of fast fashion: quick response, enhanced design, and strategic consumer behavior. Manage. Sci. $\mathbf{5 7}$ (2011) 778-795.

[8] H.L. Chan, B. Shen and Y.J. Cai, Quick response strategy with cleaner technology in a supply chain: coordination and win-win situation analysis. Int. J. Prod. Res. 56 (2018) 3397-3408.

[9] H.Y. Chen, J. Chen and Y.H. Chen, A coordination mechanism for a supply chain with demand information updating. Int. J. Prod. Econ. 103 (2006) 347-361. 
[10] A. Cheaitou and R. Cheaytou, A two-stage capacity reservation supply contract with risky supplier and forecast updating. Int. J. Prod. Econ. 209 (2019) 42-60.

[11] T.M. Choi, Inventory service target in quick response fashion retail supply chains. Serv. Sci. 8 (2016) $406-419$.

[12] T.M. Choi, Quick response in fashion supply chains with retailers having boundedly rational managers. Int. Trans. Oper. Res. 24 (2017) 891-905.

[13] T.M. Choi and P.S. Chow, Mean-variance analysis of quick response program. Int. J. Prod. Econ. 114 (2008) $456-475$.

[14] K.L. Donohue, Efficient supply chain contracts for fashion goods with forecast updating and two production modes. Manage. Sci. 46 (2000) 1397-1411.

[15] G.D. Eppen and A.V. Iyer, Backup-agreements in fashion buying-the value of upstream flexibility. Manage. Sci. 43 (1997) 1469-1608.

[16] A. Ghadge, S. Dani, R. Ojha and N. Caldwell, Using risk sharing contracts for supply chain risk mitigation: a buyer-supplier power and dependence perspective. Comput. Ind. Eng. 103 (2017) 262-270.

[17] L. Guo, The benefits of downstream information acquisition. Marketing Sci. 28 (2009) 457-471.

[18] H. Gurnani and C.S. Tang, Optimal ordering decisions with uncertain cost and demand forecast updating. Manage. Sci. 45 (1999) 1456-1462.

[19] Y.J. He, Supply risk sharing in a closed-loop supply chain. Int. J. Prod. Econ. 183 (2017) 39-52.

[20] Y.J. He and J. Zhang, Random yield risk sharing in a two-level supply chain. Int. J. Prod. Econ. 112 (2008) $769-781$.

[21] H. Huang, S.P. Sethi and H. Yan, Purchase contract management with demand forecast updating. IIE Trans. 37 (2005) $775-785$.

[22] K. Inderfurth and J. Clemens, Supply chain coordination by risk sharing contracts under random production yield and deterministic demand. OR Spectr. 36 (2014) 525-556.

[23] A.V. Iyer and M.E. Bergen, Quick response in manufacturer-retailer channels. Manage. Sci. 43 (1997) 559-570.

[24] W.S. Kim, A supply chain contract with flexibility as a risk-sharing mechanism for demand forecasting. Int. J. Syst. Sci. 44 (2013) 1134-1149.

[25] C.C. Lee and W.H.J. Chu, Who should control inventory in a supply chain? Eur. J. Oper. Res. 164 (2005) $158-172$.

[26] H. Lei, J.R. Wang, L.S. Shao and H.L. Yang, Ex post demand information sharing between differentiated suppliers and a common retailer. Int. J. Prod. Res. 58 (2020) 703-728.

[27] C.L. Li and P. Kouvelis, Flexible and risk-sharing supply contracts under price uncertainty. Manage. Sci. 45 (1999) $1289-1462$.

[28] Y.T. Lin and A. Parlakturk, Quick response under competition. Prod. Oper. Manage. 21 (2012) 518-533.

[29] W.H. Liu, X.Y. Liu and X. Li, The two-stage batch ordering strategy of logistics service capacity with demand update. Transp. Res. Part E: Logistics Transp. Rev. 83 (2015) 65-89.

[30] J.W. Pratt, Risk aversion in the small and in the large. Econometrica 32 (1964) 122-136.

[31] M. Reimann, Accurate response with refurbished consumer returns. Decis. Sci. 47 (2015) 31-59.

[32] S.P. Sethi, H.M. Yan and H.Q. Zhang, Quantity flexibility contract: optimal decisions with information updates. Decis. Sci. 35 (2004) 691-712.

[33] B. Shen, T.M. Choi and S. Minner, A review on supply chain contracting with information considerations: information updating and information asymmetry. Int. J. Prod. Res. 57 (2019) 4898-4936.

[34] Y. Shi, X.L. Guo and Y.G. Yu, Dynamic warehouse size, planning with demand forecast and contract flexibility. Int. J. Prod. Res. 56 (2018) 1313-1325.

[35] J. Spengler, Vertical integration and antitrust policy. Journal of Political Economy 58 (1950) 347-352.

[36] S.Y. Tang and P. Kouvelis, Pay-back-revenue-sharing contract in coordinating supply chains with random yield. Prod. Oper. Manage. 23 (2014) 2089-2102.

[37] A.A. Tsay, The quantity flexibility contract and supplier-customer incentives. Manage. Sci. 45 (1999) 1289-1462.

[38] J. von Neumann and O. Morgenstern, Theory of Games and Economic Behavior. seconded. Princeton University Press, Princeton (1953).

[39] T. Wakolbinger and J.M. Cruz, Supply chain disruption risk management through strategic information acquisition and sharing and risk-sharing contracts. Int. J. Prod. Res. 49 (2011) 4063-4084.

[40] X. Wang and L. Liu, Coordination in a retailer-led supply chain through option contract. Int. J. Prod. Econ. 110 (2007) $115-127$.

[41] Q.Z. Wang and D.B. Tsao, Supply contract with bidirectional options: the buyer's perspective. Int. J. Prod. Econ. 101 (2006) $30-52$.

[42] J.H. Wu, Quantity flexibility contracts under Bayesian updating. Comput. Oper. Res. 32 (2005) 1267-1288.

[43] Y.S. Xia, K. Ramachandran and H. Gurnani, Sharing demand and supply risk in a supply chain. IIE Trans. 43 (2011) 451-469.

[44] T.J. Xiao and D.Q. Yang, Risk sharing and information revelation mechanism of a one-manufacturer and one-retailer supply chain facing an integrated competitor. Eur. J. Oper. Res. 196 (2009) 1076-1085.

[45] X.M. Yan and Y. Wang, A newsvendor model with capital constraint and demand forecast update. Int. J. Prod. Res. 52 (2014) 5021-5040.

[46] S.A. Yang and J.R. Birge, Trade credit, risk sharing, and inventory financing portfolios. Manage. Sci. 64 (2018) 3469-3970.

[47] D.Q. Yang, T.J. Xiao, T.M. Choi and T.C.E. Cheng, Optimal reservation pricing strategy for a fashion supply chain with forecast update and asymmetric cost information. Int. J. Prod. Res. 56 (2018) 1960-1981. 
[48] M. Zare, M. Esmaeili and Y.J. He, Implications of risk-sharing strategies on supply chains with multiple retailers and under random yield. Int. J. Prod. Econ. 216 (2019) 413-424.

[49] Y.X. Zhao, S.Y. Wang, T.C.E. Cheng, X.Q. Yang and Z.M. Huang, Coordination of supply chains by option contracts: a cooperative game theory approach. Eur. J. Oper. Res. 207 (2010) 668-675.

[50] M.M. Zheng, M. Shunko, N. Gavirneni, Y. Shu and K. Wu, Reactive production with preprocessing restriction in supply chains with forecast updates. IISE Trans. 51 (2019) 1402-1436.

[51] Y.W. Zhou and S.D. Wang, Supply chain coordination for newsvendor-type products with two ordering opportunities and demand information update. J. Oper. Res. Soc. 63 (2012) 1655-1678. 